Aus dem Fachbereich Medizin

der Johann Wolfgang Goethe-Universität

Frankfurt am Main

betreut an der

Klinik für Dermatologie, Venerologie und Allergologie

Direktor: Prof. Dr. Roland Kaufmann

Digital unterstütztes Peer-Feedback

im Dermatologie-Praktikum

\begin{abstract}
Dissertation
zur Erlangung des Doktorgrades der Medizin

des Fachbereichs Medizin

der Johann Wolfgang Goethe-Universität

Frankfurt am Main
\end{abstract}

vorgelegt von

Daniel Manuel Chavez Barahona

aus Ica - Peru

Frankfurt am Main, 2020 
Dekan:

Referent/in:

Korreferent/in:

Tag der mündlichen Prüfung:
Prof. Dr. Stefan Zeuzem

Prof. Dr. Falk Ochsendorf

Prof. Dr. Eva Herrmann

19.08.2021 


\section{INHALTSVERZEICHNIS}

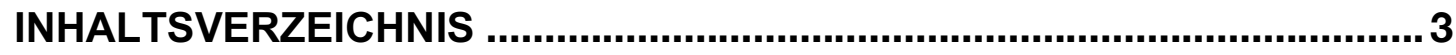

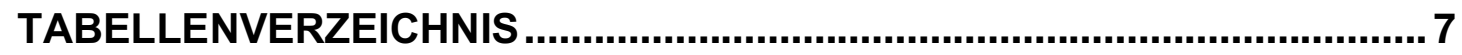

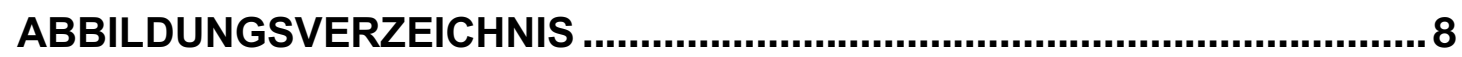

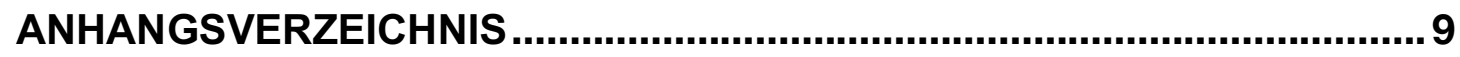

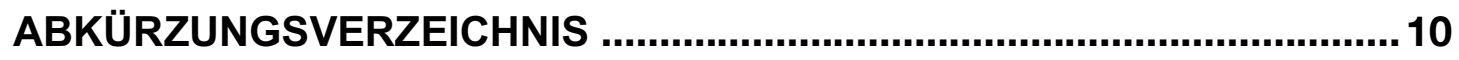

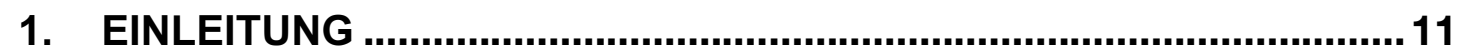

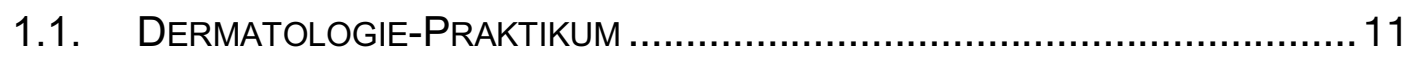

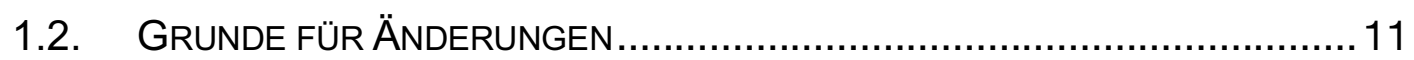

1.3. LITERATUR ZUM GEGENSEITIGEN FEEDBACK....................................12

1.4. FRAGESTELLUNG DER DOKTORARBEIT .......................................... 13

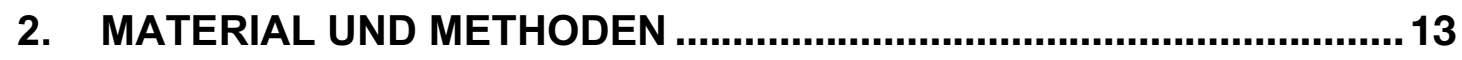

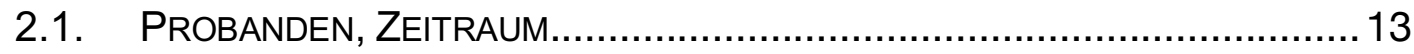

2.2. StANDARD-Ablauf des PRAKTIKUMS FÜr DeRMATOLOGIE UND

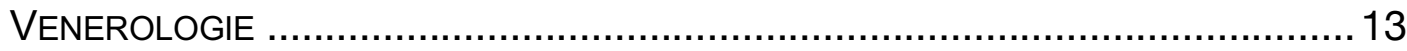

2.3. KONTROLL- UND EXPERIMENTALGRUPPE...........................................16

2.4. ABLAUF DER UNTERSUCHUNG .................................................... 16

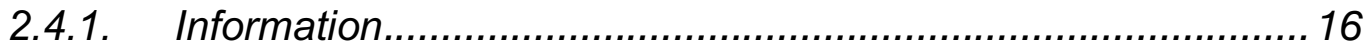

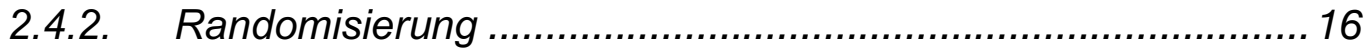

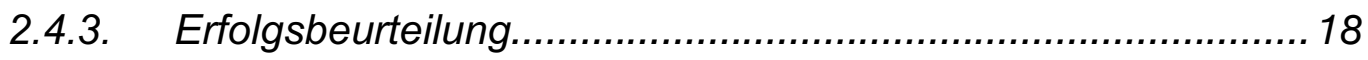

2.4.3.1. Primäres Studienziel: Elektronische Umsetzung .................. 18

2.4.3.2. Sekundäres Studienziel: Ergebnisse der Abschlussklausur

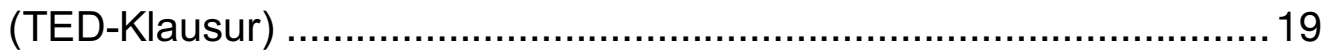

2.4.3.3. Sekundäres Studienziel: Epikrise-Abschlussfall ....................19

2.5. Ablauf DEs DeRMATOlogie KURSES IN DER EXPERIMENTELLEN GRUPPE.

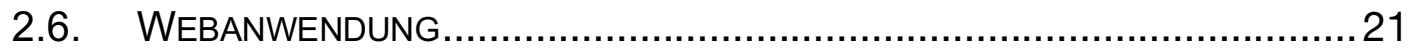

2.6.1. Verwendete Technische Materialen ......................................21

2.6.1.1. Beschreibung der Datenbank .............................................22 


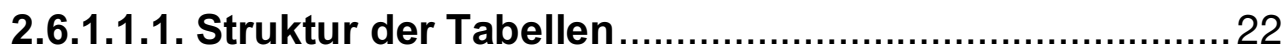

Tabelle: Daten der Studierenden ..............................................22

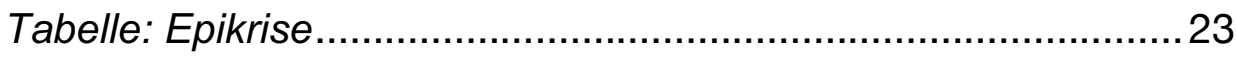

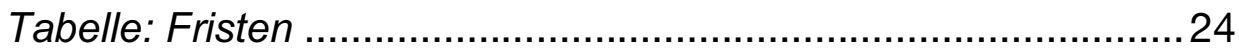

Tabelle: Abgabe Status der Epikrisen und Korrekturen ................24

2.6.1.1.2. Exportierbarkeit der Daten aus der Datenbank............24

2.6.2. Beschreibung der Phasen zur Eintragung der Epikrise und

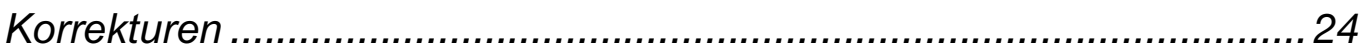

2.6.3. Umsetzung des Algorithmus für die Verteilung der Epikrisen in

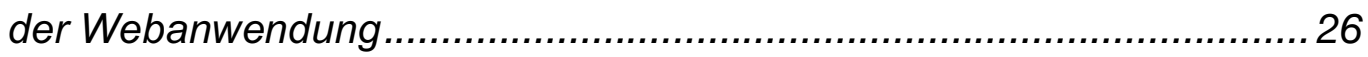

2.6.4. Beschreibung der Webanwendung ....................................... 28

2.6.4.1. Gliederung der Webanwendung ………….......................28

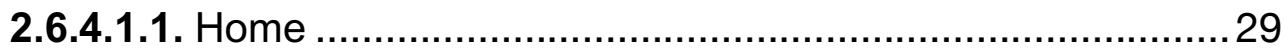

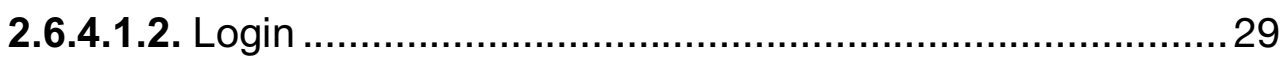

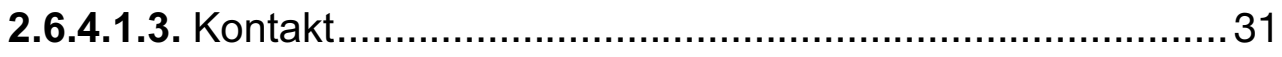

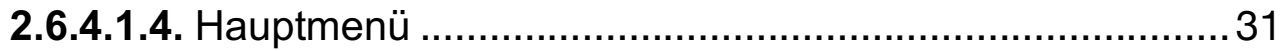

2.6.4.1.5. Information ........................................................... 35

2.6.4.1.6. Druckversion meiner Epikrise ........................................ 35

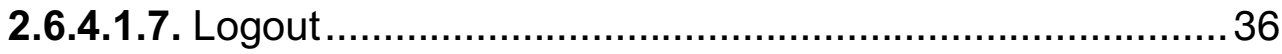

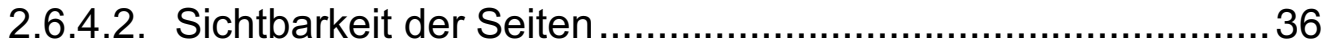

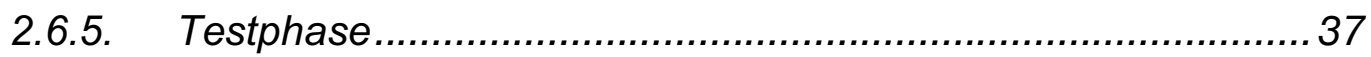

2.6.6. Verfügbarkeit der Daten für die Studierenden ......................... 38

2.7. BEWERTUNG DURCH DIE STUDIERENDEN ......................................... 38

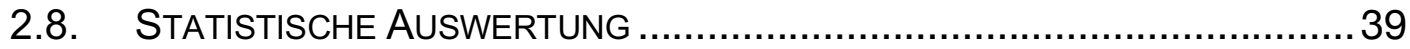

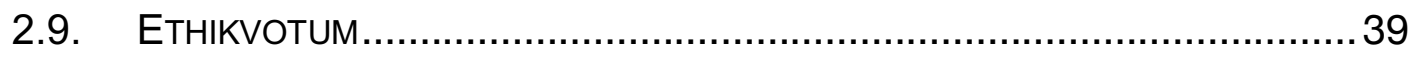

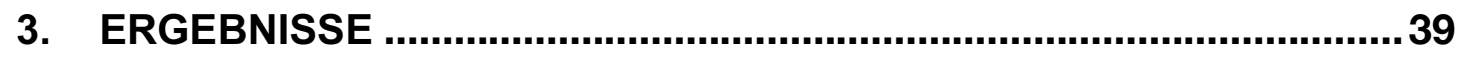

3.1. EleKTRONISCHE UMSETZUNG DES Gegenseitigen FEedBACKS ............39

3.2. TEILNAHME AN DER ABSCHLUSSKLAUSUR (TED-KLAUSUR) ....................40

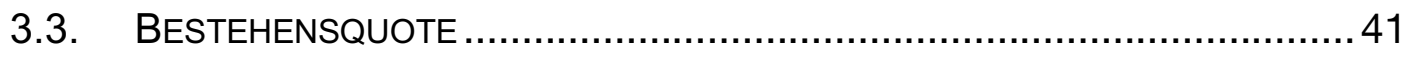

3.4. VERGLEICH EXPERIMENTALGRUPPE UND KONTROLLGRUPPE ................. 42

3.5. TeILNAHMEBEREITSCHAFT AM GEGENSEITIGEN FEEDBACK ....................43

3.6. PunKte bei deR TeILnAHME Am Gegenseitigen FeEdBACK ….............. 44 


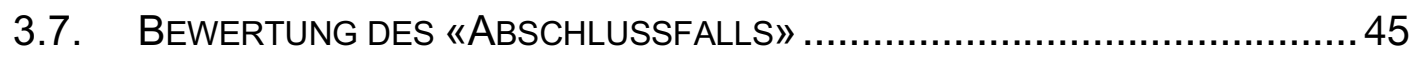

3.7.1. Erreichte Punktzahl bei der Befundbeschreibung ................... 45

3.8. BEWERTUNG DES EPIKRISE FEEDBACK SYSTEMS DURCH DIE

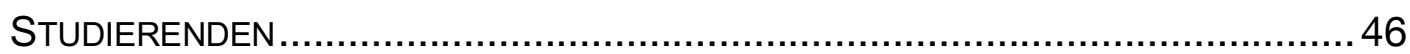

3.8.1. Selbst-Einschätzung der Verbesserung durch die Studierenden.

3.8.2. Bewertung des gegenseitigen Feedbacks durch die

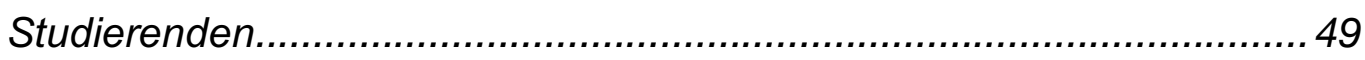

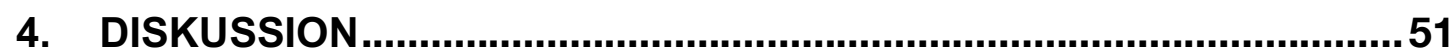

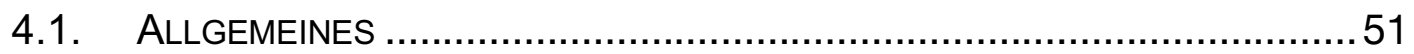

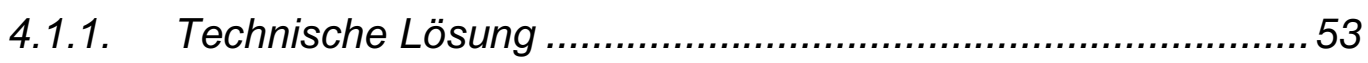

4.2. PrimÄRES STUdienZIeL: ElekTRONISCHE UMSETZUNG DES

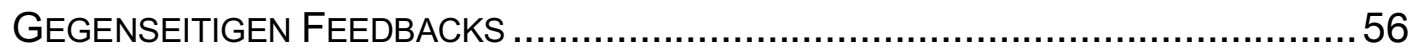

4.2.1. Anforderungen an die Webanwendung ................................5 57

4.2.2. Voraussetzungen der Webanwendung in Bezug auf die

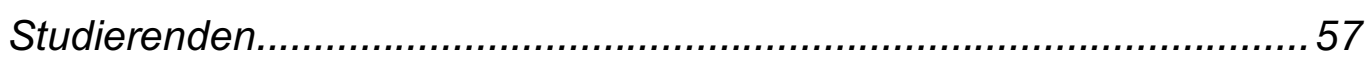

4.2.3. Voraussetzungen der Webanwendung in Bezug auf den

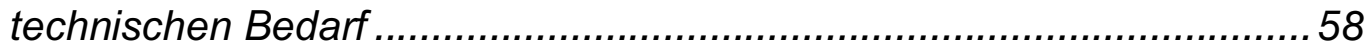

Belastbarkeit in Bezug auf Datenübertragung:..................................58

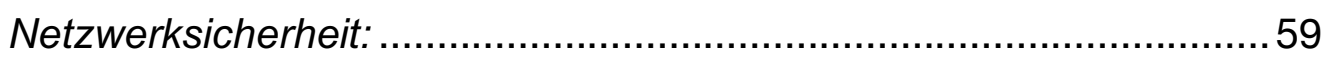

Speicherung und Datenbank: ............................................................ 59

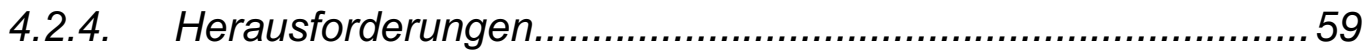

4.2.5. Entscheidung für eine Webanwendung.................................. 60

4.2.5.1. Hardware der Webanwendung: Server................................. 60

Entscheidung für einen externen Webserver: ...........................60

Gewährleistung einer einwandfreien Datenübertragung:...........61

Spiegelserver als Sicherheitsserver:........................................ 61

4.2.5.2. Software: Serversoftware, Programmiersprache und

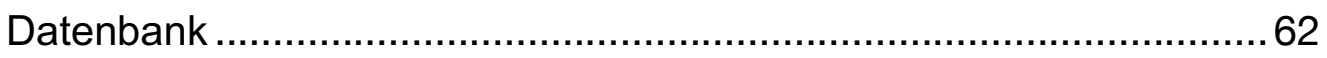

Serversoftware und Programmiersprache: .............................. 62

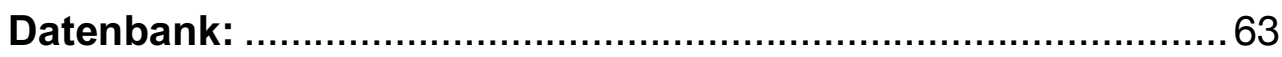

4.2.6. Testphase der Webanwendung........................................... 64 
4.2.7. Einsatz Online ............................................................... 65

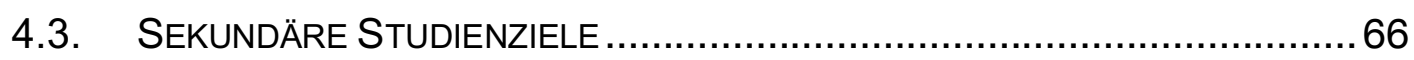

4.3.1. Mitarbeit der Experimentalgruppe .....................................66

4.3.2. Abschlussklausur (TED- Klausur).....................................67 67

4.3.3. Epikrise-Abschlussfall................................................. 67

4.3.4. Fragebogen zur Selbsteinschätzung und Bewertung des gegenseitigen Feedbacks ........................................................... 68

4.3.4.1. Selbsteinschätzung durch die Studierenden.......................69

4.3.4.2. Bewertung des gegenseitigen Feedbacks durch die Studierenden ....................................................................... 69

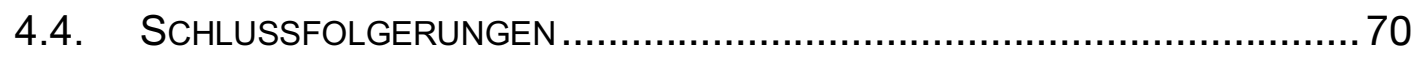

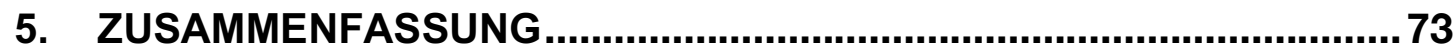

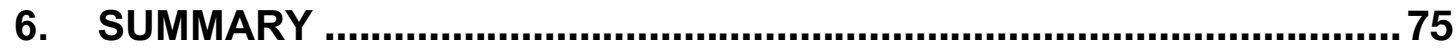

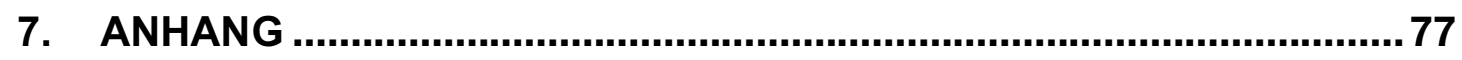

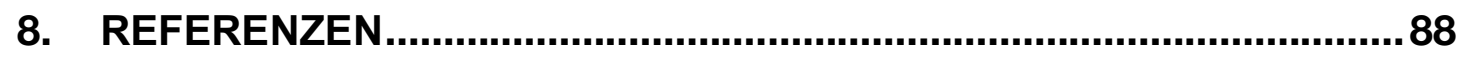

9. SCHRIFTLICHE ERKLÄRUNG......................................................93

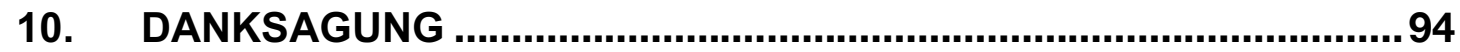

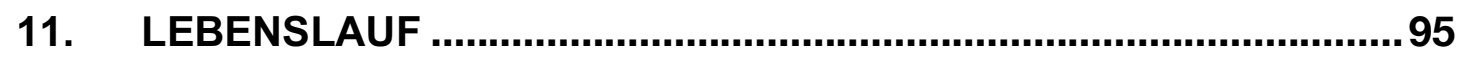




\section{TABELLENVERZEICHNIS}

Tabelle 1: Randomisierung der Studierenden....................................... 18

Tabelle 2: Sichtbarkeit der verschiedenen Seiten .................................... 37

Tabelle 3: Durchschnittliche Punktzahl der Fragen zur Selbsteinschätzung in

Experimental- und Kontrollgruppe ............................................... 47 


\section{ABBILDUNGSVERZEICHNIS}

Abbildung 1: Stundenplan des Praktikums Dermatologie und Venerologie... 14 Abbildung 2: Anzahl der Studierenden in Kontroll- und Experimentalgruppe nach Randomisierung und bei Verwechslung einer Gruppe ........................ 17

Abbildung 3: Zeitplan der Experimentalgruppe inklusive Fristen ................... 20

Abbildung 4: Versand und Korrektur der Epikrisen ...................................... 21

Abbildung 5: Verschiedene Phasen und Fristen ......................................... 26

Abbildung 6: Algorithmus zur Verteilung der Epikrisen ................................ 27

Abbildung 7: Gliederung der Webanwendung ………............................. 28

Abbildung 8: Willkommensseite der Webanwendung .................................. 29

Abbildung 9: Screenshot der Registrierungsseite ...................................... 30

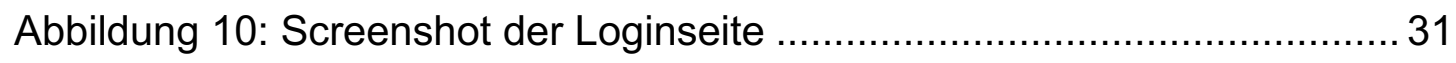

Abbildung 11: Hinterlegte Kontakt-Daten auf der Webanwendung................ 31

Abbildung 12: Screenshot des Hauptmenüs auf der Webanwendung............ 32

Abbildung 13: Epikrise-Formular der Webanwendung anhand eines Beispiels

Abbildung 14: Screenshot des Korrekturformulars anhand eines Beispiels .. 34

Abbildung 15: Screenshot der Information auf der Webanwendung..... 35

Abbildung 16: Vorschau über die Druckversion der Epikrise anhand eines

Beispiels 36

Abbildung 17: Randomisierung und Teilnahme der Studierenden an der TED-

Klausur

Abbildung 18: Bestehensquote in Experimental- und Kontrollgruppe.

Abbildung 19: Vergleich der Punktzahl zwischen Kontroll- und

Experimentalgruppe

Abbildung 20: Teilnahme am gegenseitigen Feedback

Abbildung 22: Vergleich der durchschnittlichen Punktzahl der Fragen zur

Selbsteinschätzung in Experimental- und Kontrollgruppe

Abbildung 23: Bewertung des gegenseitigen Feedbacks durch die

Studierenden 50 


\section{ANHANGSVERZEICHNIS}

Anhang 1: Formblatt der Epikrise während der Hospitation und für den

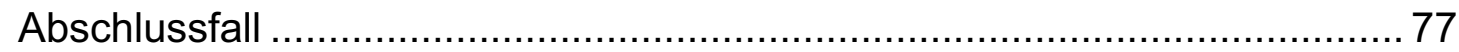

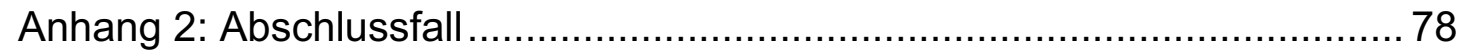

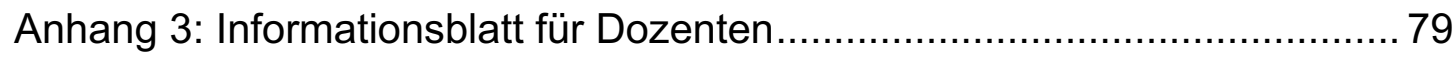

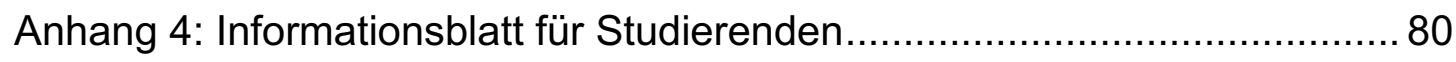

Anhang 5: Zeiträume und Friste (Deadlines) ……..................................... 81

Anhang 6: Anleitung zum Anfertigen der Epikrise Online .............................. 82

Anhang 7: Fragebogen für die Epikrise Abschlussfall................................. 83

Anhang 8: Fragebogen zur Selbsteinschätzung (Kontrollgruppe) ................. 84

Anhang 9: Fragebogen zur Selbsteinschätzung (Experimentalgruppe)......... 86 


\section{ABKÜRZUNGSVERZEICHNIS}

EBM Evidenz-basierte Medizin

EG Experimentalgruppe

HTML Hypertext Markup Language

ID Identifikationsnummer

KW Kalenderwoche

KG Kontrollgruppe

MC Multiple Choice

PHP Hypertext Preprocessor, ursprünglich Personal Home

Page Tools

TED-Klausur Tele-Dialog-System Klausur 


\section{EINLEITUNG}

Eine Untersuchung im Fachgebiet der Dermatologie besteht aus einer kurzen Anamnese mit anschließender Inspektion der Haut. Damit man den Hautbefund korrekt beschreiben kann, sollte man sowohl Lokalisation, Form, Verteilung als auch Farbe der Effloreszenzen genau benennen. Je mehr Erfahrung der Betrachter hat, desto schneller kann die Diagnose durch Erkennung von Mustern gestellt werden, während sich bei Anfängern diese Fähigkeit erst entwickeln muss [1]. Die Theorie wird im „Propädeutikum Dermatologie" im ersten sowie in der Hauptvorlesung im 4. klinischen Semester vermittelt.

Die Fähigkeit zur korrekten Befundbeschreibung und Mustererkennung muss dann durch aktives, bewusstes Üben („deliberate practice“) verbessert werden [2, 3]. Lernen anhand aktiver Übung setzt aber voraus, dass die Lernenden ein relevantes Feedback erhalten, damit sie sich verbessern können [2]. Dies erfolgt im 5. klinischen Semester im Blockpraktikum Dermatologie.

\subsection{Dermatologie-Praktikum}

Das Dermatologie-Block-Praktikum an der Universität Frankfurt ist ein wesentlicher Teil des Dermatologie-Unterrichtes. Hierbei werden die Studierenden des 5. klinischen Semesters der Humanmedizin während einer Woche sowohl in interaktiven Seminaren, Hospitationen, Unterricht am Krankenbett als auch mittels E-Learning-Elementen (Übungen zur Befundbeschreibung, Hausaufgaben, Bearbeitung virtueller Patientenfälle) unterrichtet. Am Ende des Kurses wird jeweils eine Abschlussklausur mit MCFragen durchgeführt.

Zum Bestehen des Kurses sind eine aktive Mitarbeit, erfolgreiche Befundbeschreibungen, die Erstellung eine Epikrise sowie das Bestehen der Abschlussklausur notwendig. Bei Nicht-Erfolg kann diese bis maximal zweimal wiederholt werden.

\subsection{GRUNDE FÜR ÄNDERUNGEN}

Seit Jahren wird versucht, das Dermatologie-Praktikum zu verbessern, indem man nach und nach verschiedene Elemente eingebaut hat. Mit der Einführung 
von freiwilligen Hausaufgaben im Hörsaal wurde die Vorbereitungszeit der Studierenden vor Beginn des Kurses signifikant verbessert [4]. Durch fallbasierte Präsenzlehre und Online-Fallpräsentationen wurde das Praktikum strukturiert und die Abhängigkeit von zufällig verfügbaren Patienten beseitigt [5]. Die Online-Fallpräsentationen erfolgten mittels der sogenannten „virtuellen Poliklinik“. Zudem wurde dadurch neben der Eigenaktivität auch ein professionelles Verhalten im Sinne einer Selbstreflexion gefördert [5]. Ein wesentliches Problem war, dass die Studierenden nach wie vor Schwierigkeiten hatten, eine korrekte dermatologische Befundbeschreibung anzufertigen. Eine Doktorarbeit aus dem Jahr 2007 beschäftigte sich mit der Fragestellung, ob es durch Umformulierung der Fragen in der virtuellen Poliklinik sowie der Bearbeitung entweder vor oder nach dem Präsenzunterricht zu einer Verbesserung der Befundbeschreibung kommt. Es zeigte sich, dass auch die virtuelle Poliklinik leider keine durchgängige Verbesserung der Fähigkeiten hinsichtlich der Befundbeschreibung bewirkte [5].

Aus diesem Grund beschäftigt sich die vorliegende Arbeit mit der Frage, wie die Fähigkeiten zur Befundbeschreibung verbessert werden könnten. Eine Überlegung hierbei war, dass ein gegenseitiges Feedback durch die Studierenden zu einem besseren Lerneffekt führen könnte als ein Feedback durch die Dozenten. In einer früheren Untersuchung hatte sich gezeigt dass ein persönliches Feedback vom Tutor an die Studierenden auch in Kleingruppen schwierig durchführbar war [4]. Die Überlegung war daher, dass dieses gegenseitige Feedback am besten digital umgesetzt werden könnte, z.B. mittels einer Lernplattform in Form einer Webanwendung. Dies vereinfacht es, Daten wie Befundbeschreibungen zu verteilen, einzusammeln, zu analysieren und zu administrieren. Deshalb bestand die Fragestellung bzw. das Hauptziel dieser Arbeit darin, eine solche Webanwendung zu entwickeln, die unter diesen Voraussetzungen einwandfrei funktioniert sowie auch im Unterricht eingesetzt werden kann.

\subsection{LITERATUR ZUM GEGENSEITIGEN FEEDBACK}

Insgesamt zeigte sich in der Literatur, dass Peer-assistierte Lehrmethoden zu geringen bis mittleren positiven Auswirkungen bezüglich Sozialverhalten, 
Selbsteinschätzung und Verhalten führen. Dies wurde beispielsweise in einer Meta-Analyse von Ginsburg-Block et al. nachgewiesen [6]. Eine Arbeit von Max Field et al. aus dem Jahr 2007 zeigte eine große Akzeptanz der Studierenden hinsichtlich eines gegenseitigen Feedback in derselben Kohorte [7].

Diese positiven Erfahrungen sprachen dafür, ein gegenseitiges FeedbackTool zu entwickeln. Bis zum Anfang dieser Untersuchung waren keine Studien publiziert, welche ein anonymes, gegenseitiges Feedback-zwischen den Studierenden in Bezug auf eine medizinische Befundbeschreibung untersucht hatten.

\subsection{FRAGESTELLUNG DER DOKTORARBEIT}

Die vorliegende Studie sollte die folgenden Fragen beantworten:

- Ist die elektronische Umsetzung eines gegenseitigen Feedbacks von Epikrisen möglich?

- Verbessert ein gegenseitiges Feedback von Epikrisen die Prüfungsleistung in einer Abschlussklausur?

- Verbessert dieses gegenseitige Feedback die Fähigkeit zur korrekten Befundbeschreibung?

- Wie beurteilen die Studierenden diesen Ansatz?

\section{MATERIAL und Methoden}

\subsection{Probanden, Zeitraum}

Es wurden prospektiv alle Studierenden in die Studie einbezogen, die im Sommersemester 2014 am «Praktikum Dermatologie und Venerologie» teilnahmen. Die Studierenden befanden sich im 4. oder im 5. klinischen Semester des Medizinstudiums. Insgesamt waren 181 Studierende für den Kurs angemeldet.

\subsection{Standard-ABlauf des PRAKTIKums FÜR DERMATOlogiE UND VENEROLOGIE}

Das 5-tägige Blockpraktikum «Dermatologie und Venerologie» ist standardisiert und erfolgt gemäß dem in Abbildung 1 dargestellten Schema. In 
jeder Woche betreut ein anderer Oberarzt der dermatologischen Klinik als Tutor die Studierenden während der gesamten Woche.

\begin{tabular}{|c|c|c|c|c|c|}
\hline Uhrzeit & Mo & $\mathrm{Di}$ & $\mathrm{Mi}$ & Do & $\mathrm{Fr}$ \\
\hline $8.00-12.00$ & Hospitation & Studierende 1-5 & Studierende $6-10$ & Studierende 11-15 & Studierende $16-20$ \\
\hline $\begin{array}{l}\text { Haupt- } \\
\text { thema }\end{array}$ & Tumore & Allergie & Infektion & Haut und System & $\begin{array}{c}\text { Sexuell übertragbare } \\
\text { Erkrankungen }\end{array}$ \\
\hline & & \begin{tabular}{|l|} 
Fakultativ: \\
Virtuelle Poliklinik Fall
\end{tabular} & & & \begin{tabular}{|l|} 
Fakultativ: \\
Virtuelle Poliklinik Fall
\end{tabular} \\
\hline 8.00 & Ablaufbesprechung & Eigenstudium & Eigenstudium & Eigenstudium & Eigenstudium \\
\hline \multirow{2}{*}{10.00} & \begin{tabular}{|l|} 
Effloreszensen/ \\
Befundbeschreibung
\end{tabular} & \multirow{3}{*}{$\begin{array}{l}\text { E-Learning Hausaufgabe } \\
\text { Virtuelle Poliklinik }\end{array}$} & \multirow{3}{*}{$\begin{array}{l}\text { E-Learning Hausaufgabe } \\
\text { Virtuelle Poliklinik }\end{array}$} & \multirow{3}{*}{$\begin{array}{l}\text { E-Learning Hausaufgabe } \\
\text { Virtuelle Poliklinik }\end{array}$} & \multirow{3}{*}{$\begin{array}{l}\text { E-Learning Hausaufgabe } \\
\text { Virtuelle Poliklinik } \\
\text { Online-Evaluation }\end{array}$} \\
\hline & Lokaltherapie & & & & \\
\hline 13.00 & $\begin{array}{l}\text { Lokaltherapie/banale } \\
\text { Hautveränderung }\end{array}$ & & & & \\
\hline 14.00 & Fall & Fall Hausaufgabe & Studentenfall & Studentenfall & Studentenfall \\
\hline 16.00 & Patientenuntersuchung & Patientenuntersuchung & Patientenuntersuchung & Patientenuntersuchung & Abschlussbesprechung \\
\hline
\end{tabular}

\section{Abbildung 1: Stundenplan des Praktikums Dermatologie und Venerologie}

Das Blockpraktikum ist zeitlich sehr eng strukturiert. Jeweils 5 Studierende hospitieren Dienstag bis Freitagvormittag. In der restlichen Zeit erfolgen das Eigenstudium und die Bearbeitung der Hausaufgaben.

Jeder Studierende hospitiert an einem Vormittag der Woche (Dienstag, Mittwoch, Donnerstag oder Freitag) für 4 Stunden in einem zugeteilten Bereich der Hautklinik (onkologisch-chirurgische Station, konservativallergologische Station, Hochschulambulanz, Allergie-Ambulanz oder im OP). Während dieser Hospitation untersucht jeder Studierende einen Patienten, welcher je nach aktueller Verfügbarkeit zugeteilt wird. Anschließend erstellt der Studierende eine Epikrise über ebendiesen Patienten nach einem standardisierten Schema (siehe Anhang 1). Die Epikrise wird auf einem vorgefertigten Formular dokumentiert und umfasst die Punkte:

- Anamnese

- Hautbefund

- Verdachtsdiagnosen

- diagnostisches Procedere

- Auswirkungen der Erkrankung auf den Patienten

Diese Epikrise wird am selben Tag schriftlich beim jeweiligen Tutor abgegeben und anhand eines standardisierten Schemas beurteilt und mit Punkten bewertet. In diesem Schema werden die Haupt-/Verdachtsdiagnose und 2 Differentialdiagnosen genannt und erklärt sowie die Auswirkung der Hauterkrankung auf den Patienten beschrieben (siehe Anhang 1).

Die Punkte-Vergabe erfolgt nach folgenden Vorgaben: 
- Befund: 5 Punkte

- Haupt/Verdachtsdiagnose (1): 1 Punkt

- Differentialdiagnose (2): 1 Punkt

- Differentialdiagnose (3): 1 Punkt

- Zur Klärung von (1): 1 Punkt

- Zur Klärung von (2): 1 Punkt

- Zur Klärung von (3): 1 Punkt

- Auswirkung der Hauterkrankung auf den Patienten: 3 Punkte

Aus Zeitgründen wurde die Beurteilung durch den Tutor bis zum jetzigen Zeitpunkt erst nach dem Kurs durchgeführt, so dass keine Rückmeldung über die konkrete Leistung an die Studierenden erfolgte.

Als Erfolgskontrolle dient eine Klausur. Diese wird mittels eines TED-Systems (=Tele-Dialog-System, auch Audience Response System oder Classroom Response System) durchgeführt.

Das TED-System ist ein Live-Abstimmungssystem, bei welchem die Studierenden sich mittels einer Fernbedienung ( 5 verschiedene Tasten/Knöpfe) für die richtige Antwort entscheiden können. Bei jeder Frage gab es 5 Antwortmöglichkeiten, nur eine Antwort war jeweils richtig. Bei der Klausur waren jeweils 5 Aufsichtspersonen anwesend, um die Möglichkeit des "Abschreibens" bzw. Absprechens zu minimieren.

In der Abschlussklausur des Dermatologie-Praktikums wurden die Teilnehmer von 3 - 4 Kurswochen an einem gemeinsamen Termin in einem großen Hörsaal anhand von $20 \mathrm{MC}-$-Fragen geprüft.

Pro Semester erfolgten 4 Klausuren mit jeweils unterschiedlichen Fragen. Um die Klausur und damit das Dermatologie-Praktikum zu bestehen, musste jeder Studierende mindestens 10 von 20 Punkten, also 50\% der Punkte erreichen. Termine der Abschlussklausur für die Teilnehmer im Sommersemester 2014: Kurs 1: Kalenderwochen 26, 27, 28, 30 am Freitag, den 25.07.2014 Kurs 2: Kalenderwochen 31, 32, 33, 34 (und nach-/wiederholende Studierende vom 1. Kurs) am Freitag, den 22.08.2014 Kurs 3: Kalenderwochen 35, 36, 37, 38 (und nach-/wiederholende Studierende vom 1. und 2. Kurs) am Freitag, den 19.09.2014 
Nachklausur für wiederholende Studierende der Kurse 1-3 am Freitag, den 10.10.2014

\subsection{KONTROLL- UND EXPERIMENTALGRUPPE}

Die Kontrollgruppe waren die Studierenden, die den normalen Kursablauf absolvierten. Die Experimentalgruppe waren die Studierende, die am gegenseitigen Feedback mittels des neu entwickelten Programms teilnehmen sollten.

\subsection{ABLAUf DER UNTERSUCHUNG}

\subsubsection{INFORMATION}

Alle Tutoren des Dermatologie-Kurses wurden im Vorfeld schriftlich und mündlich über die Studie informiert. Sie waren damit einverstanden und bekamen auch die entsprechenden Materialien (Infoblätter, Bögen, etc.) für die Kontroll- bzw. die Interventionsgruppe zugeteilt. Die Materialien für die Kontrollgruppe waren die, für den Kurs genutzten standardisierten Unterrichtsmaterialien.

Die Studierenden im Standardkurs erhielten keine speziellen Informationen. Die Studierenden in der experimentellen Gruppe wurden informiert, dass ein neuer Ansatz im Kurs verwendet wird (siehe Anhang 4: Informationsblatt für Studierenden).

\subsubsection{RANDOMISIERUNG}

181 Studierende wurden durch das Dekanat wochenweise in 12

Studierenden-Gruppen eingeteilt. Die 12 Gruppen wurden für die Studie mittels Zufallszahlen in Kontroll- und Experimentalgruppe randomisiert. Hierfür wurde ein Online-Tool der Firma GraphPad verwendet, namens Random number generator (https://www.graphpad.com/quickcalcs/randomN1.cfm).

Vor Beginn des Kurses bestanden die Kontrollgruppe aus 89 Probanden und die Experimentalgruppe aus 92 Probanden. In der Kalenderwoche 35 hat der Tutor jedoch versehentlich die Kontrollgruppe mit einer Experimentalgruppe verwechselt. Dadurch erhielten diese Studierenden Zugang zum gegenseitigen Feedback (Experimentalgruppe). Gemäß einer «As-Treated- 
Analyse» wurden diese 13 Studierenden der Kalenderwoche 35 als

Experimentalgruppe ausgewertet. Somit gab es bei der Auswertung 76

Studierende in der Kontrollgruppe und 105 Studierende in der

Experimentalgruppe (siehe Abbildung 2 und Tabelle 1).

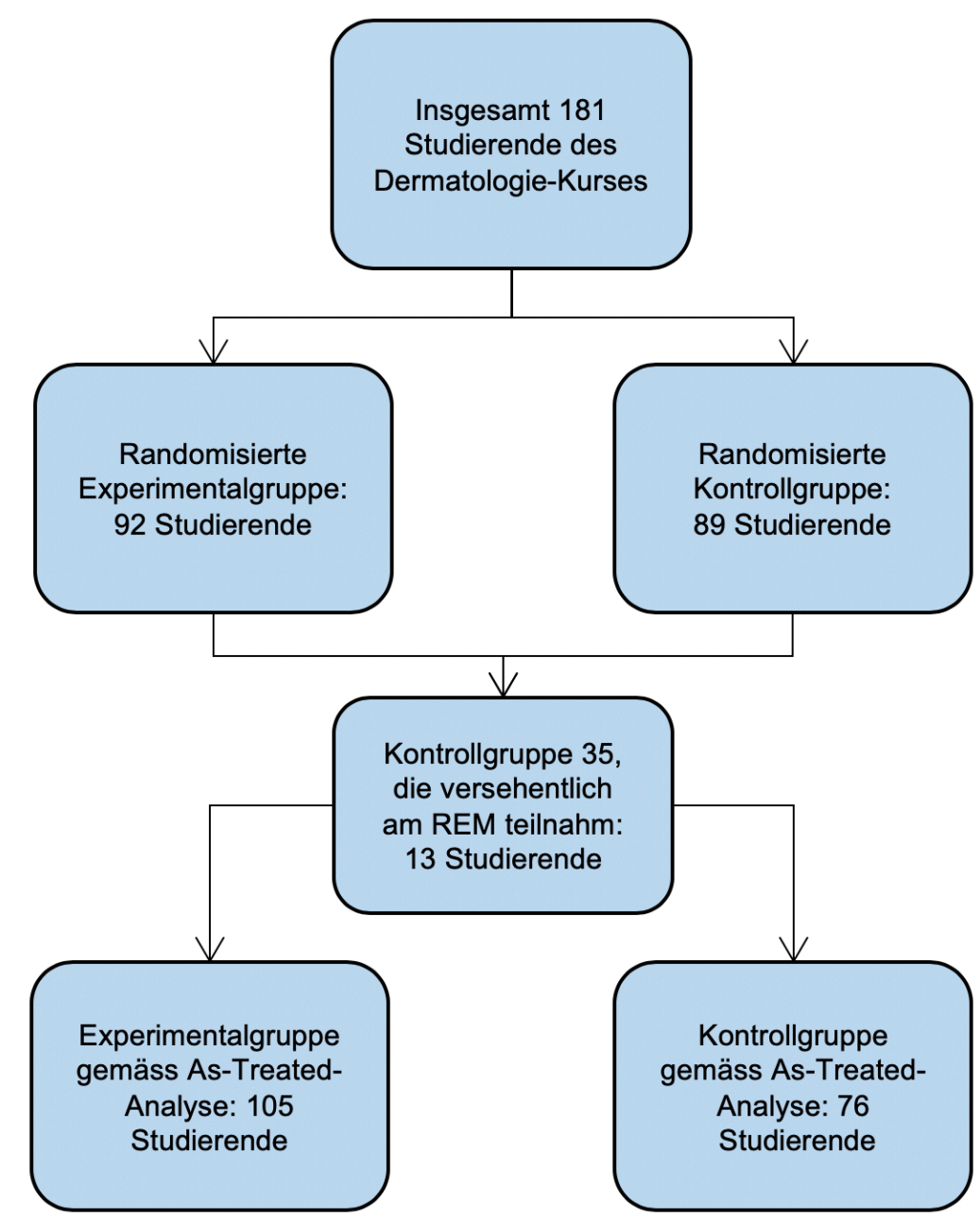

Abbildung 2: Anzahl der Studierenden in Kontroll- und Experimentalgruppe nach Randomisierung und bei Verwechslung einer Gruppe

Durch eine Verwechslung eines Tutors erhielt die Kontrollgruppe in Kalenderwoche 35

Zugang zu der Online-Lernplattform und wurde gemäß der „As Treated-Analyse“ als

Experimentalgruppe ausgewertet. 


\begin{tabular}{|r|l|l|}
\hline KW & Randomisiert & As treated \\
\hline $\mathbf{2 6}$ & Kontrollgruppe & Kontrollgruppe \\
\hline $\mathbf{2 7}$ & Kontrollgruppe & Kontrollgruppe \\
\hline $\mathbf{2 8}$ & Experimentalgruppe & Experimentalgruppe \\
\hline $\mathbf{2 9}$ & Kein Kurs & Kein Kurs \\
\hline $\mathbf{3 0}$ & Kontrollgruppe & Kontrollgruppe \\
\hline $\mathbf{3 1}$ & Experimentalgruppe & Experimentalgruppe \\
\hline $\mathbf{3 2}$ & Kontrollgruppe & Kontrollgruppe \\
\hline $\mathbf{3 3}$ & Kontrollgruppe & Kontrollgruppe \\
\hline $\mathbf{3 4}$ & Experimentalgruppe & Experimentalgruppe \\
\hline $\mathbf{3 5}$ & Kontrollgruppe & Experimentalgruppe \\
\hline $\mathbf{3 6}$ & Experimentalgruppe & Experimentalgruppe \\
\hline $\mathbf{3 7}$ & Experimentalgruppe & Experimentalgruppe \\
\hline $\mathbf{3 8}$ & Experimentalgruppe & Experimentalgruppe \\
\hline & & \\
\hline
\end{tabular}

\section{Tabelle 1: Randomisierung der Studierenden}

Die Studierenden wurden wochenweise der Kontroll- und Experimentalgruppe zugeordnet. In der Woche 29 fand kein Kurs statt. Durch die Verwechslung eines Tutors wurde die Gruppe aus KW 35 von einer Kontrollgruppe zur Experimentalgruppe und gemäß der "As treated Analyse" so ausgewertet. KW - Kalenderwoche (bezogen auf das Jahr 2014).

\subsubsection{ERFOLGSBEURTEILUNG}

\subsubsection{Primäres Studienziel: Elektronische Umsetzung}

Als primäres Studienziel wurde der einwandfreie Verlauf einer elektronischen Umsetzung des gegenseitigen Feedbacks festgelegt. 


\subsubsection{Sekundäres Studienziel: Ergebnisse der Abschlussklausur (TED-Klausur)}

Als erstes sekundäres Studienziel wurde die erreichte Punktzahl in der TEDKlausur analysiert (s.u. Null-Hypothese).

\subsubsection{Sekundäres Studienziel: Epikrise-Abschlussfall}

Als zweites sekundäres Studienziel wurde die Punktzahl bei der Bewertung des „Abschlussfalls“ ausgewertet.

Diesen Epikrise-Abschlussfall mussten alle Studierenden am Ende des Dermatologie-Praktikums (Freitag) bearbeiten, unabhängig davon ob sie in Kontroll- oder Experimentalgruppe waren. Es handelte sich um einen virtuellen Patienten. Jeder Studierende erhielt eine Beschreibung des Patienten (siehe Anhang 1), zudem erfolgte eine Präsentation der Hautveränderungen als projizierte Bilder. Zu diesem Patienten sollte in gleicher Art und Weise wie während der Hospitation eine erneute Epikrise verfasst werden. Diese wurde vom Doktoranden anhand des oben beschriebenen Epikrise-Auswertungsschemas beurteilt.

\subsection{Ablauf des DeRmatologie KuRses IN DER EXPERIMENTELLEN GRUPPE}

Die Studierenden der Experimentalgruppe verfassten wie im Standard-Kurs eine schriftliche Epikrise über den zugeteilten Hospitation-Patienten. Diese wurde einerseits wie bisher beim jeweiligen Tutor abgegeben. Zusätzlich mussten die Studierenden aber auch an inrem Hospitationstag bis spätestens 23:59 Uhr diese Epikrise in der Webanwendung eintragen, um gegenseitiges Feedback zu erhalten.

Nach dem Eintragen versendete das System diese Epikrise an zwei zufällig ausgewählte andere Studierende. Nach Ablauf der angegebenen Frist wurden die Epikrisen für die jeweils zugeteilten Studierenden zur Korrektur freigegeben. Die Korrekturen erfolgten anonym.

Die Studierenden wurden bereits am Anfang des Kurses über den genauen Ablauf, den Umfang der Epikrise und die Fristen bzw. Deadlines informiert. Die Fristen sind im Anhang 5 beschrieben. Die Studierenden wurden zusätzlich vom Doktoranden per Email, SMS oder telefonisch daran erinnert, 
dass die zu beurteilende Epikrise in das Programm einzutragen war, falls sie noch keine Einträge gemacht hatten. Zum besseren Verständnis der jeweiligen Fristen (Eintragen der Epikrise, Korrekturen) bei den unterschiedlichen Hospitation-Gruppen hilft die Abbildung 3.

\begin{tabular}{|c|c|c|c|c|c|c|c|}
\hline Uhrzeit & Mo & \multicolumn{3}{|c|}{$\mathrm{Di}$} & \multicolumn{3}{|c|}{ Mi } \\
\hline $8.00-12.00$ & & \multirow{5}{*}{$\begin{array}{c}\text { Registrierung } \\
\text { Online für die } \\
\text { Studenten }\end{array}$} & $\begin{array}{c}\text { Hospitationstag } \\
\text { Gruppe A }\end{array}$ & \multirow{6}{*}{$\begin{array}{c}\text { Eintragen der } \\
\text { Epikrise Online } \\
\text { Gruppe A }\end{array}$} & $\begin{array}{c}\text { Hospitationstag } \\
\text { Gruppe B }\end{array}$ & \multirow{6}{*}{$\begin{array}{c}\text { Eintragen der } \\
\text { Epikrise Online } \\
\text { Gruppe B }\end{array}$} & \multirow{2}{*}{$\begin{array}{c}\text { Korrektur der } \\
\text { Epikrise A }\end{array}$} \\
\hline $12.00-13.00$ & & & & & & & \\
\hline $13.00-16.00$ & & & & & & & \\
\hline $16.00-17.00$ & & & & & & & \\
\hline $17.00-20.00$ & \multirow{2}{*}{$\begin{array}{l}\text { Registrierung Online } \\
\text { für die Studenten }\end{array}$} & & & & & & \\
\hline $20.00-23.59$ & & & & & & & \\
\hline
\end{tabular}

\begin{tabular}{|c|c|c|c|c|c|c|c|}
\hline Uhrzeit & \multicolumn{3}{|c|}{ Do } & \multicolumn{3}{|c|}{$\mathrm{Fr}$} & Sa \\
\hline $8.00-12.00$ & $\begin{array}{c}\text { Hospitationstag } \\
\text { Gruppe C }\end{array}$ & \multirow{6}{*}{$\begin{array}{c}\text { Eintragen der } \\
\text { Epikrise Online } \\
\text { Gruppe C }\end{array}$} & \multirow[t]{2}{*}{$\begin{array}{c}\text { Korrektur der } \\
\text { Epikrise B }\end{array}$} & \begin{tabular}{|c|} 
Hospitationstag \\
Gruppe D \\
\end{tabular} & \multirow{6}{*}{$\begin{array}{l}\text { Eintragen der } \\
\text { Epikrise Online } \\
\text { Gruppe D }\end{array}$} & $\begin{array}{c}\text { Korrektur der } \\
\text { Epikrise C }\end{array}$ & \multirow[t]{2}{*}{$\begin{array}{c}\text { Korrektur der } \\
\text { Epikrise D }\end{array}$} \\
\hline $12.00-13.00$ & & & & & & & \\
\hline $13.00-16.00$ & & & & & & Abschlussfall & \\
\hline $16.00-17.00$ & & & & & & Epikrise & \\
\hline $17.00-20.00$ & & & & & & & \\
\hline $20.00-23.59$ & & & & & & & \\
\hline
\end{tabular}

\section{Abbildung 3: Zeitplan der Experimentalgruppe inklusive Fristen}

Am Montagabend um 17 Uhr wurde die Registrierung für die Studierenden freigeschaltet und die Anmeldung sollte bis Dienstag um 20 Uhr erfolgen. Je nachdem, an welchem Tag den Studierenden ein Patient zugeteilt worden war, wurden sie unterteilt in Gruppe A bis D. Sie mussten am selben Tag, an welchem sie die Anamnese erhoben und den Patienten untersucht hatten, auch die Epikrise online schreiben und versenden. Die Korrektur der Epikrise-Online sollte dann jeweils am Folgetag zwischen 8 Uhr und 13 Uhr erfolgen.

Insgesamt erhielt jeder Studierende somit von zwei Studierenden Feedback und bewertete auch selbst zwei fremde Epikrisen. Der prinzipielle Ablauf ist anhand der 3 Studierenden A, B und C in der Abbildung 4 dargestellt. 

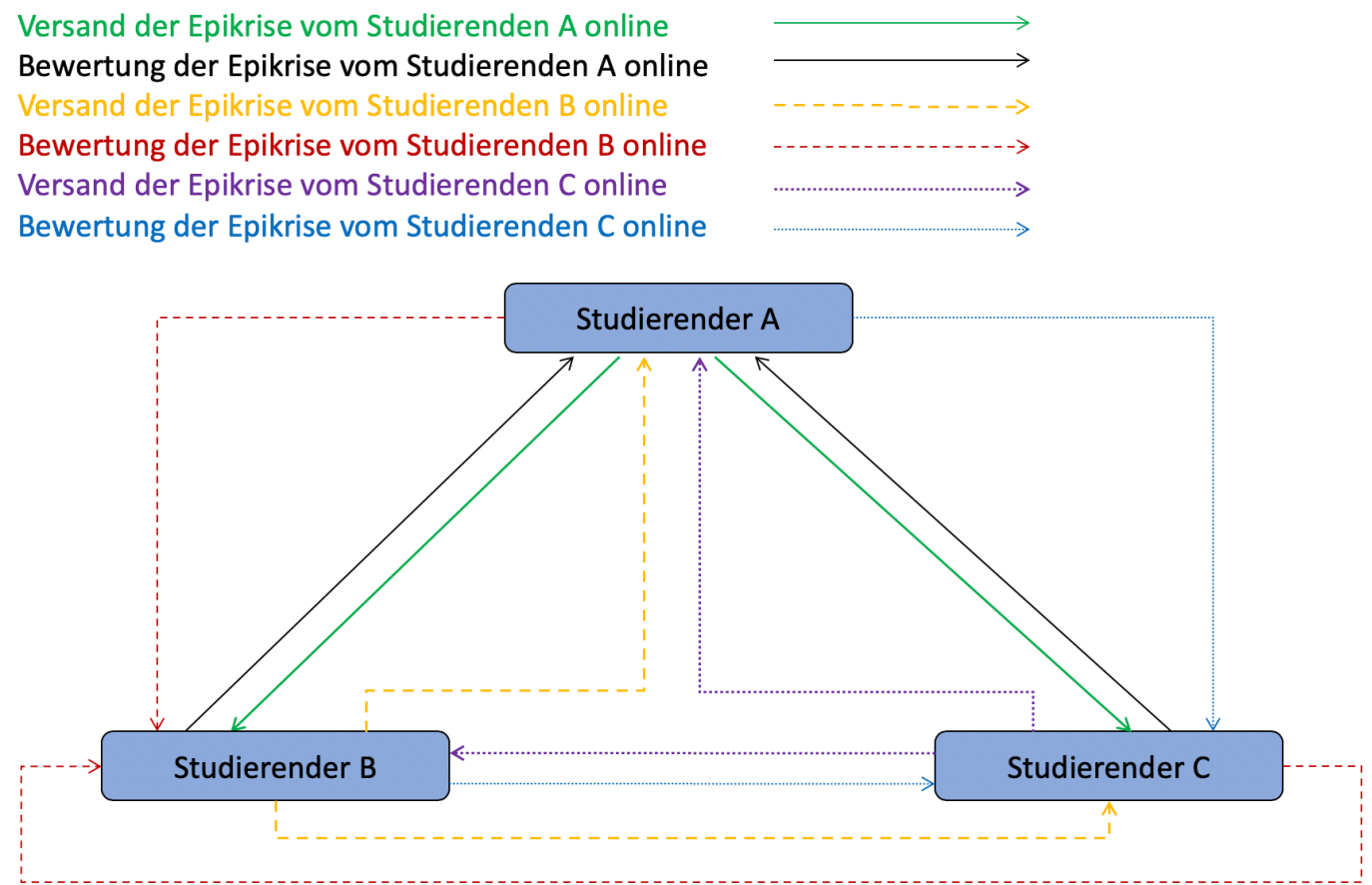

\section{Abbildung 4: Versand und Korrektur der Epikrisen}

Ein Studierender (A) schreibt eine Epikrise online. Anschließend wird diese verschickt und dabei zufällig und anonym zwei anderen Studierenden $(B, C)$ zugeteilt. Diese sollten die Epikrise anhand vorgegebener Gliederungspunkte bewerten. Nach Beendigung der Korrektur wird diese für den Studierenden $(\mathrm{A})$ wieder freigeschaltet und er kann sich die Korrekturen durchlesen und daraus lernen. Jeder Studierende soll somit idealerweise eine Epikrise schreiben und zwei fremde Epikrisen korrigieren. Außerdem erhält jeder Studierende dadurch zwei Korrekturen für seine Epikrise.

\subsection{WEBANWENDUNG}

Um den Studierenden gegenseitiges Feedback zu ermöglichen, mussten die Epikrisen anderen Studierenden zur Verfügung gestellt werden. Hierzu wurde eine Webanwendung benutzt. Die Webanwendung befindet sich weiterhin unter der folgenden URL online: http://epikrise-feedbackkonzept.de/index.php oder http://www.reciprocal-evaluation-model.de.

\subsubsection{VERWENDETE TECHNISCHE MATERIALEN}

Zur Entwicklung der Webanwendung verwendeten wir einen Webserver, welcher mit der Server-Software Apache HTTP Server $₫$ administriert wurde. Aus Sicherheitsgründen wurde ein zweiter Server verwendet, welcher als Spiegelserver diente. Die Beschreibung der Server und Sicherheitsserver 
sowie die Gründe der Verwendung werden in der Diskussion ausführlich besprochen (siehe 4.2.5.1 Hardware der Webanwendung: Server).

Für die Webanwendung wurde die Programmiersprache PHP, welche zur Erstellung von dynamischen Webseiten und Webanwendungen verwendet wird, eingesetzt. Zudem kam das Datenbankverwaltungssystem MySQL für die Datenbankentwicklung zum Einsatz. Als Software wählten wir die Plattform phpMyAdmin.

\subsubsection{Beschreibung der Datenbank}

Um eine Datenbank erstellen zu können, muss zuvor definiert werden, um welche Daten es sich handelt. In diesem Fall waren dies die Daten der Studierenden, die Fristen sowie die Daten von Epikrisen und Korrekturen. Zusätzlich wurden die Algorithmen zur Verteilung der Epikrisen in Zusammenhang mit der Datenbank erstellt.

Somit wurden 4 Tabellen in der Datenbank erstellt (Daten der Studierenden, Fristen, Daten von Epikrisen und Korrekturen).

In allen Tabellen wurden die Identifikationsnummern (ID-Nummern) in der Datenbank mit einer automatischen Funktion hinzugefügt. Mit dieser Identifikationsnummer konnten die Daten der verschiedenen Tabellen miteinander verbunden werden. Anhand der Identifikationsnummer konnte nachvollzogen werden, welcher Studierende eine bestimmte Epikrise eingetragen hatte und ob dieser Studierende Korrekturen an den Epikrisen anderer Studierender durchgeführt hatte.

\subsection{Struktur der Tabellen}

\section{Tabelle: Daten der Studierenden}

Diese Tabelle beinhaltete persönliche Informationen über die Studierenden. Das war nötig, um nachvollziehen zu können, welche Studierende korrekt am gegenseitigen Feedback teilgenommen hatten und welche Note die jeweiligen Studierenden in der Anschlussklausur erreicht hatten.

Zum Einloggen auf der Webanwendung waren ein Kennwort und ein Benutzername bzw. eine Emailadresse nötig. Die Emailadresse wurde für die Kommunikation mit den Studierenden, insbesondere zur Erinnerung an die Zeiträume der Fristen benötigt.

Somit war die Tabelle mit den Daten der Studierenden wie folgt gegliedert: 
- Identifikationsnummer

- Vorname

- Name

- Matrikelnummer

- Gruppe bzw. Kalenderwoche

- Fachsemester

- Benutzername

- Kennwort

- Email

\section{Tabelle: Epikrise}

In dieser Tabelle wurden die Informationen über die Epikrise des Hospitationstages gespeichert

- Grund der Vorstellung

- Bisheriger Verlauf

- Befund

- Haupt-/Verdachtsdiagnose (1)

- Differentialdiagnose (2)

- Differentialdiagnose (3)

- Diagnostische Maßnahmen zur Klärung der Verdachtsdiagnose

- Zur Klärung von (1)

- Zur Klärung von (2)

- Zur Klärung von (3)

- Aktueller Therapievorschlag

- Auswirkung der Hauterkrankung auf den Patienten

Da jeder dieser Unterpunkte von 2 anderen Studierenden korrigiert und bewertet werden sollte, wurden zu jedem dieser Unterpunkte noch 2 Textfelder hinzugefügt, beispielsweise:

- Korrektur 1 für Grund der Vorstellung

- Korrektur 2 für Grund der Vorstellung

Zusätzlich wurden 2 Spalten für die Identifikationsnummer der Studierenden, welche die Korrekturen gemacht hatten, hinzugefügt. 


\section{Tabelle: Fristen}

Diese Tabelle wurde erzeugt, um die entsprechenden Zeitpunkte der Fristen einzutragen. Es war wichtig, dass die Webanwendung die Fristen der Registrierung, Epikrisen-Abgaben und Korrekturen aufrufen konnte, damit die richtige Oberfläche zum richtigen Zeitpunkt angezeigt wurde. Die Daten wurden mit Datum und Uhrzeit mit Sekundengenauigkeit von dem Doktoranden eingetragen. Die jeweiligen genauen Zeitpunkte waren abhängig von Gruppe und Kalenderwoche. Die Tabelle speicherte folgende Daten:

- Registrierungsbeginn und Beginn der Eintragung der Epikrise

- Ende der Eintragung der Epikrise und Registrierungsende

- Beginn der Eintragung der Korrekturen

- Ende der Eintragung der Korrekturen

\section{Tabelle: Abgabe Status der Epikrisen und Korrekturen}

Für eine automatisierte zufällige Verteilung der zu korrigierenden Epikrisen an die Studierenden war eine weitere Tabelle nötig, in welcher die Information gespeichert wurde, ob ein Studierender eine Epikrise geschrieben hatte und ob diese an einen weiteren Studierenden zur Korrektur weitergeleitet worden war.

Mit dieser Tabelle war es zudem einfacher nachzuvollziehen wer bereits eine Epikrise oder Korrektur eingetragen hatte. Anhand dieser Information konnten Studierende somit auch gegebenenfalls rechtzeitig vor Ablauf der Fristen erinnert werden.

\subsection{Exportierbarkeit der Daten aus der Datenbank}

Die Webanwendung phpMyAdmin ist in der Lage, alle Tabellen der Datenbank als eine CSV (comma-separated values) Datei zu exportieren. Diese Datei kann von den meisten Tabellenkalkulationsprogrammen in tabellarischer Form gelesen und bearbeitet werden.

\subsubsection{BESCHREIBUNG DER PHASEN ZUR EINTRAGUNG DER EPIKRISE UND KORREKTUREN}

Der Algorithmus für das gegenseitige Feedback wurde bereits unter dem Punkt «Ablauf des Dermatologie Kurses in der experimentellen Gruppe beschrieben». 
Die technische Umsetzung wurde wie folgt entwickelt:

- Nach der Registrierung konnte die Epikrise eingetragen werden.

- Die Epikrise durfte bis zu der Frist «Ende der Eintragung der Epikrise» eingetragen werden. Nach diesem Zeitpunkt konnte die Epikrise von dem Studierenden zwar weiter aufgerufen aber nicht mehr bearbeitet werden.

- Nun erfolgte eine randomisierte Zuteilung der Epikrisen zu je zwei anderen Studierenden. Anschließend konnten die Studierenden die Korrekturen eintragen.

- Nach Ablauf der Frist «Ende der Eintragung der Korrekturen» konnten keine Korrekturen mehr gemacht werden. Die erfolgten Korrekturen wurden anschließend nur dem jeweiligen Studierenden angezeigt, der die Epikrise geschrieben hatte.

Die folgende Grafik vereinfacht das Verständnis dieses Ablaufes: 


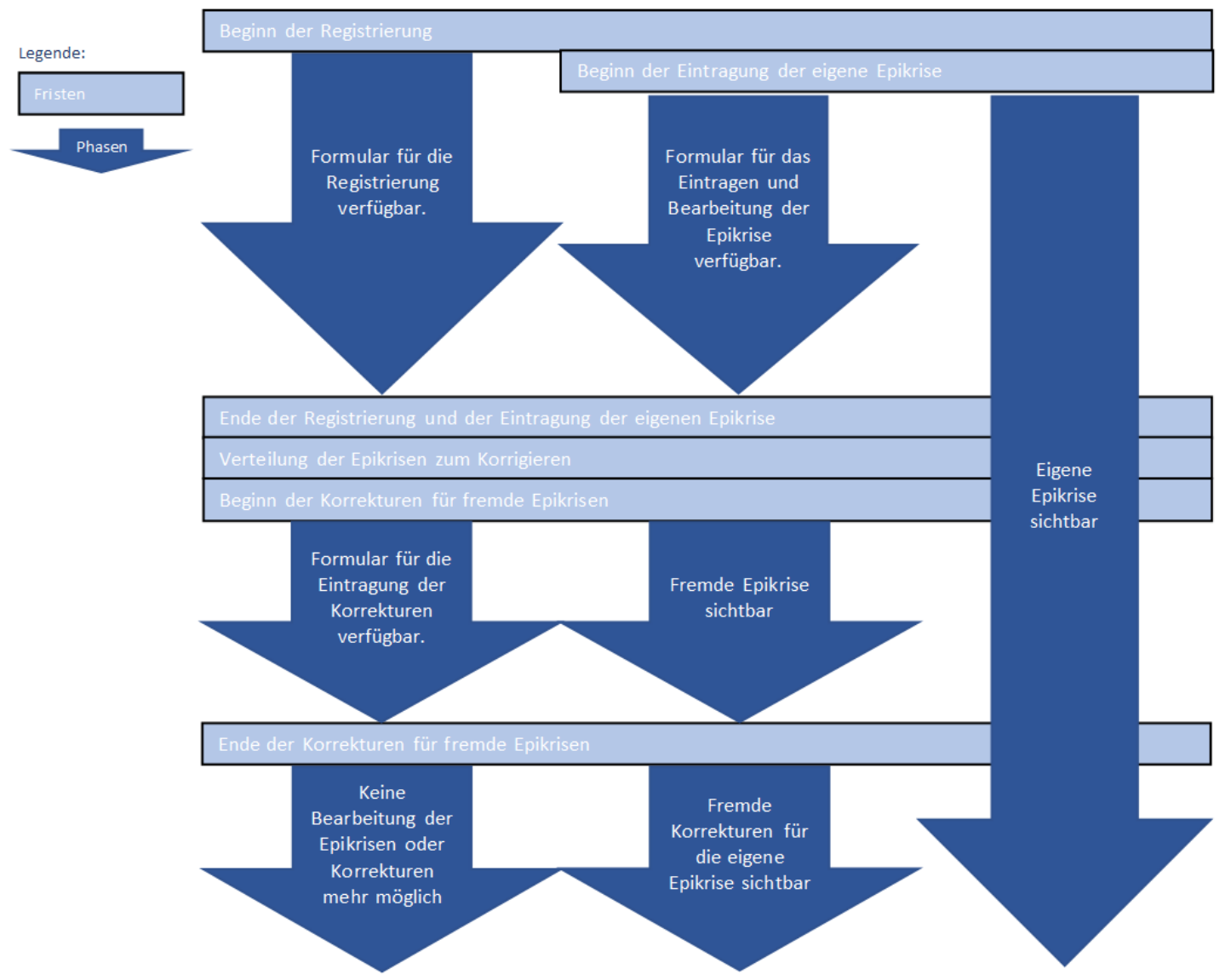

\section{Abbildung 5: Verschiedene Phasen und Fristen}

Diese Abbildung zeigt den zeitlichen Ablauf vom Beginn der Registrierung bis zum Erhalt der Korrekturen. Nach der Registrierung kann die Epikrise geschrieben werden bis zu einer bestimmten Frist. Anschließend erhält man eine fremde Epikrise zum Korrigieren. Die eigene Epikrise bleibt zu jedem Zeitpunkt sichtbar, kann jedoch nach Ablauf der Frist nicht mehr bearbeitet werden. Nach Ablauf der Frist bzgl. den Korrekturen besteht kein Zugriff mehr auf die fremde Epikrise, dafür werden jetzt bei der eigenen Epikrise die Korrekturen angezeigt. Es können zu diesem Zeitpunkt weder die eigene Epikrise noch die Korrekturen bearbeitet werden.

\subsubsection{UMSETZUNG DES ALGORITHMUS FÜR DIE VERTEILUNG DER EPIKRISEN IN DER WEBANWENDUNG}

Die folgende Abbildung stellt dar, wie das Konzept für die Verteilung programmiert wurde. Die Programmierung erfolgte in PHP. Die Fristen konnten aus der entsprechenden Tabelle entnommen werden. 
Jedes Mal, wenn ein Studierender sich eingeloggt hat, ist der folgende Prozess abgelaufen:

\section{Algorithmus für die Verteilung der Epikrisen}

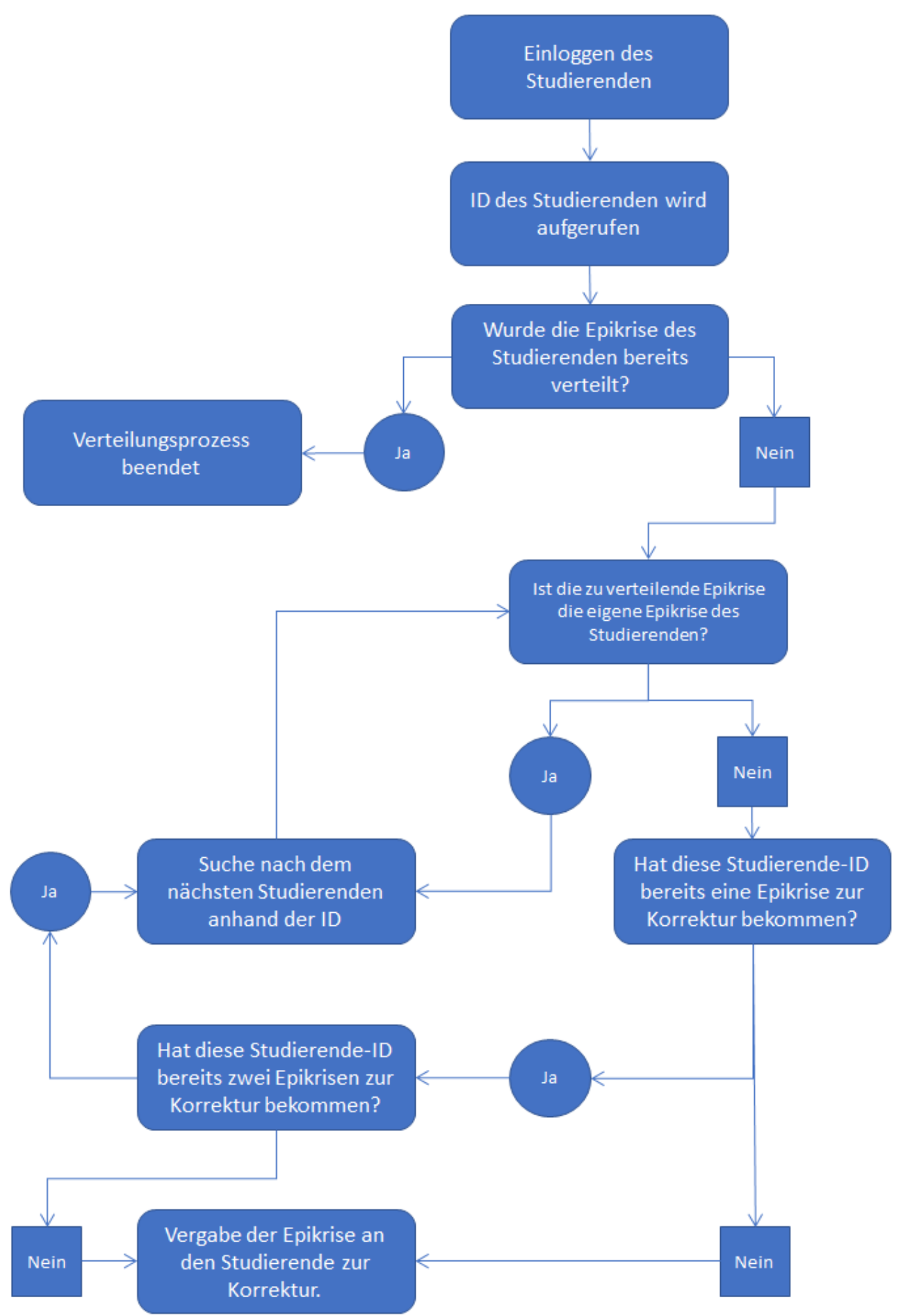

\section{Abbildung 6: Algorithmus zur Verteilung der Epikrisen}

Beim Einloggen auf der Webanwendung wurde die ID des Studierenden aufgerufen. Das Programm überprüfte beim Verteilen einer Epikrise zunächst anhand der ID, dass eine 
Epikrise nicht dem jeweiligen Verfasser der Epikrise zum Korrigieren zugeteilt wurde. Anschließend wurde überprüft ob der Studierende, der anhand der ID ausgewählt worden war, schon eine Epikrise zur Korrektur erhalten hatte. War dies nicht der Fall, wurde die Epikrise diesem Studierenden zugeteilt. Sollte dieser Studierende schon eine Epikrise zur Korrektur erhalten haben, überprüfte das Programm, ob es nur eine oder schon 2 Epikrisen waren. Falls dieser Studierende erst eine Epikrise erhalten hatte, wurde ihm die aktuelle Epikrise zugeteilt. Falls dieser Studierende jedoch schon 2 Epikrisen zum Korrigieren erhalten hatte, dann suchte das Programm den nächsten Studierenden. Auch hier wurde mittels ID zunächst wieder überprüft, dass es sich nicht um den Verfasser der Epikrise handelte.

\subsubsection{BESCHREIBUNG DER WEBANWENDUNG}

\subsubsection{Gliederung der Webanwendung}

Die Webanwendung wurde wie folgt gegliedert:

\section{Gliederung der Webapplikation (Online-Plattform)}

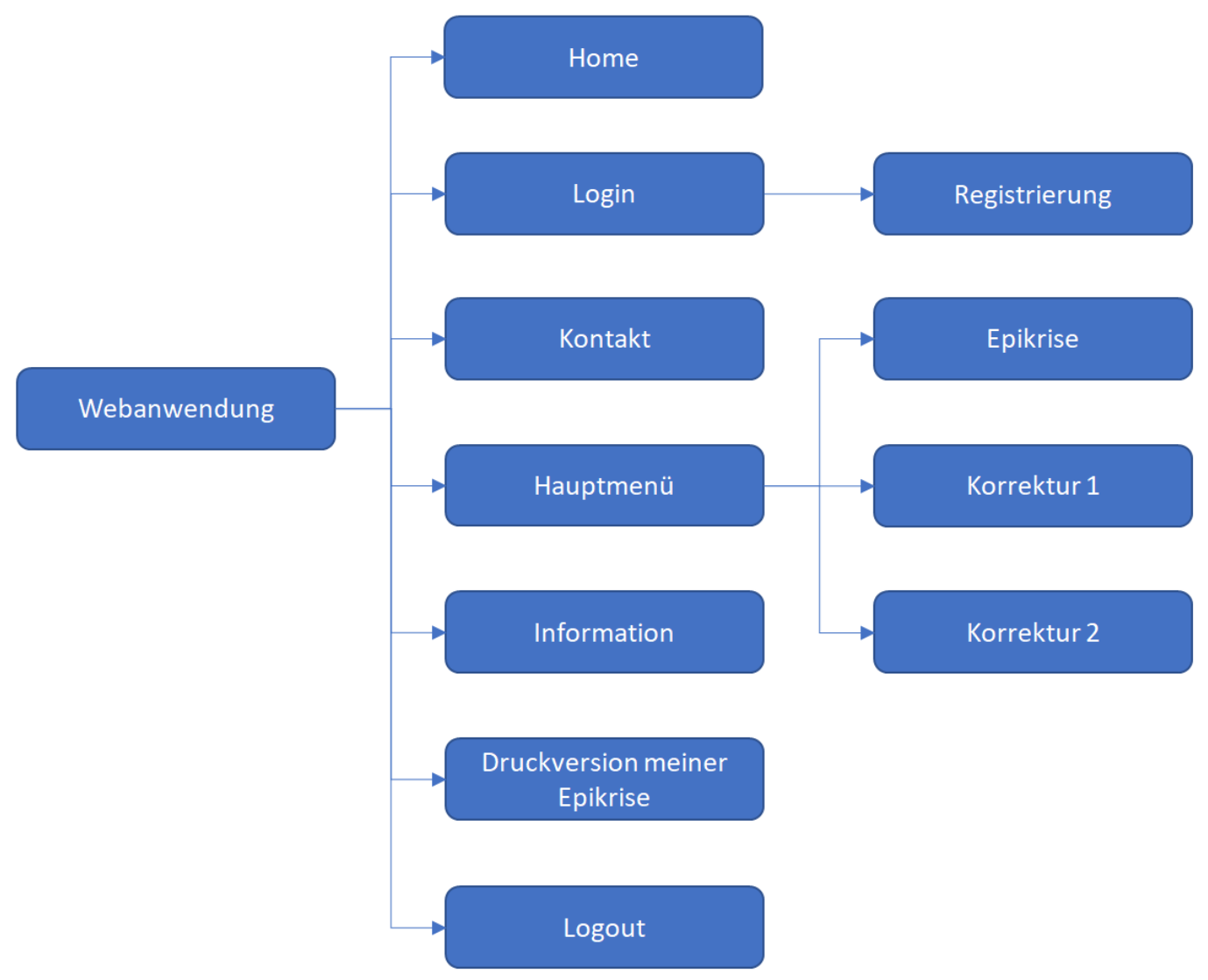

\section{Abbildung 7: Gliederung der Webanwendung}

In dieser Abbildung sind in der mittleren Spalte alle Seiten der Webseite und deren Zugehörigkeit dargestellt. Durch Klicken auf den Button Hauptmenü gelangt man zur Epikrise und den beiden Korrekturen. 


\subsubsection{Home}

Es handelt sich um eine Willkommensseite.

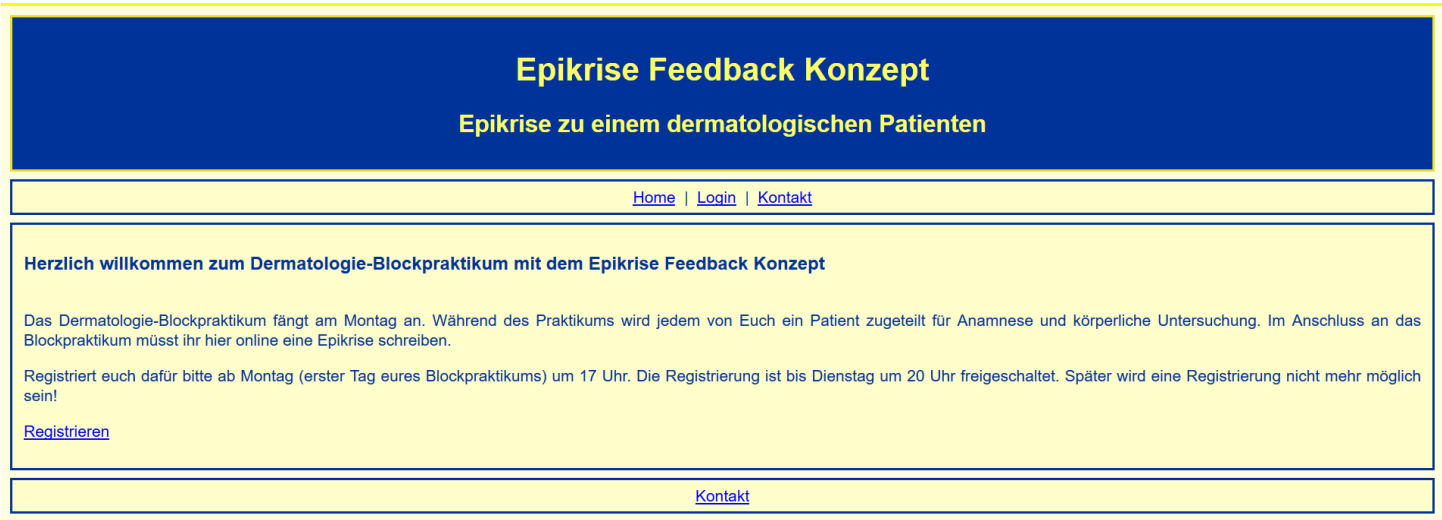

\section{Abbildung 8: Willkommensseite der Webanwendung}

Unter der Webseite http://epikrise-feedback-konzept.de/index.php oder http://www.reciprocalevaluation-model.de erhalten die Studierenden Zugang zur Webanwendung für das gegenseitige Feedback. Auf der hier gezeigten Startseite werden die Studierenden willkommen geheißen und aufgefordert, sich zu registrieren, so dass später anhand der Matrikelnummern eine Zuordnung zu den Klausurergebnissen möglich war.

\subsection{Login}

Vor dem ersten Login war eine Registrierung notwendig. Unterhalb des Feldes für den Login befand sich der Link zur Registrierung. Bei der Registrierung mussten folgende Pflichtfelder ausgefüllt werden: Vorname, Nachname, Emailadresse, Matrikelnummer, Tag der Patientenuntersuchung (Hospitationstag), Fachsemester, Benutzername, Kennwort, Wiederholung des Kennworts. Mittels einer selbstprogrammierten Anwendung wurde die Seite so gestaltet, dass eine Registrierung nur möglich war, wenn alle Pflichtfelder ausgefüllt wurden. Es wurde bewusst eine doppelte Eingabe des Kennwortes verlangt, um Probleme durch Tippfehler im Kennwort zu vermeiden. Bei fehlender Übereinstimmung der beiden Kennwörter, wäre eine Fehlermeldung erschienen. Eine Registrierung mit einer bereits vorher registrierten Matrikelnummer, Emailadresse oder Benutzernamen war nicht möglich, um Mehrfach-Registrierungen eines Studierenden zu vermeiden. Auch in diesen Fällen wäre es eine Fehlermeldung erschienen. Nach der Registrierung konnten die persönlichen Daten nicht mehr verändert werden. Zur nachträglichen Korrektur von Daten hätte sich der Studierende an den Administrator (Doktorand) wenden müssen. Die Kontaktdaten (Email) 
des Administrators waren auf der Webanwendung hinterlegt. Auch bei Vergessen des Passwortes war eine Wiederherstellung des Kennworts nur nach Kontakt des Administrators möglich.

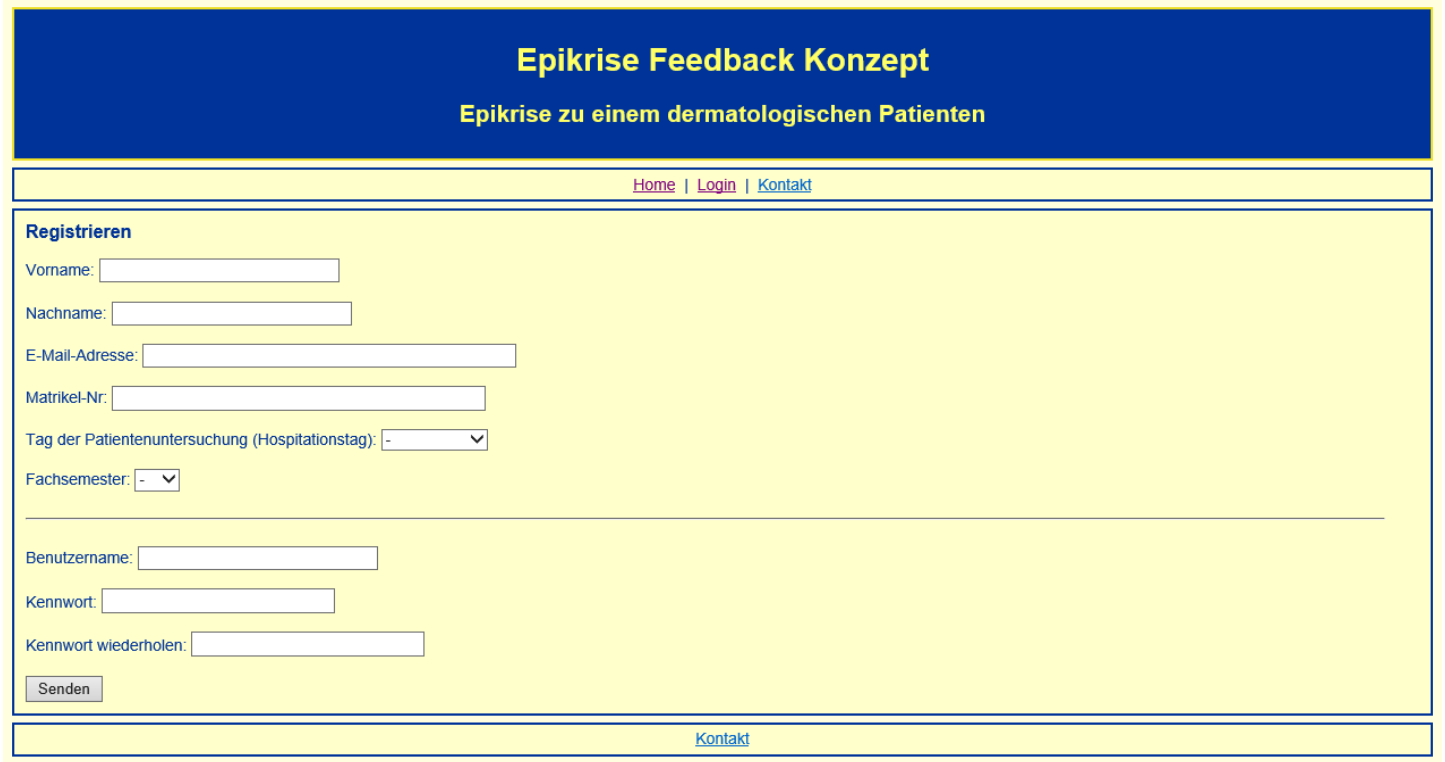

\section{Abbildung 9: Screenshot der Registrierungsseite}

Die Studierenden mussten zur Registrierung Vorname, Nachname, Emailadresse, Matrikelnummer, Hospitationstag und Fachsemester angeben. Zudem sollten sie einen Benutzernamen und ein Kennwort auswählen, welches sie bei der Registrierung zur Sicherheit doppelt eingeben mussten.

Die Daten der Studierenden, insbesondere Namen und Matrikelnummern wurden regelmäßig vom Administrator überprüft anhand von Teilnehmerlisten des Dermatologie-Praktikums. Es gab diesbezüglich keine falsche Eintragung von Daten.

Nach erfolgter Registrierung konnten sich die Studierenden auf der LoginSeite mit einem Benutzernamen und dem, zuvor bei der Registrierung gewähltes Kennwort auf der Webanwendung anmelden. 


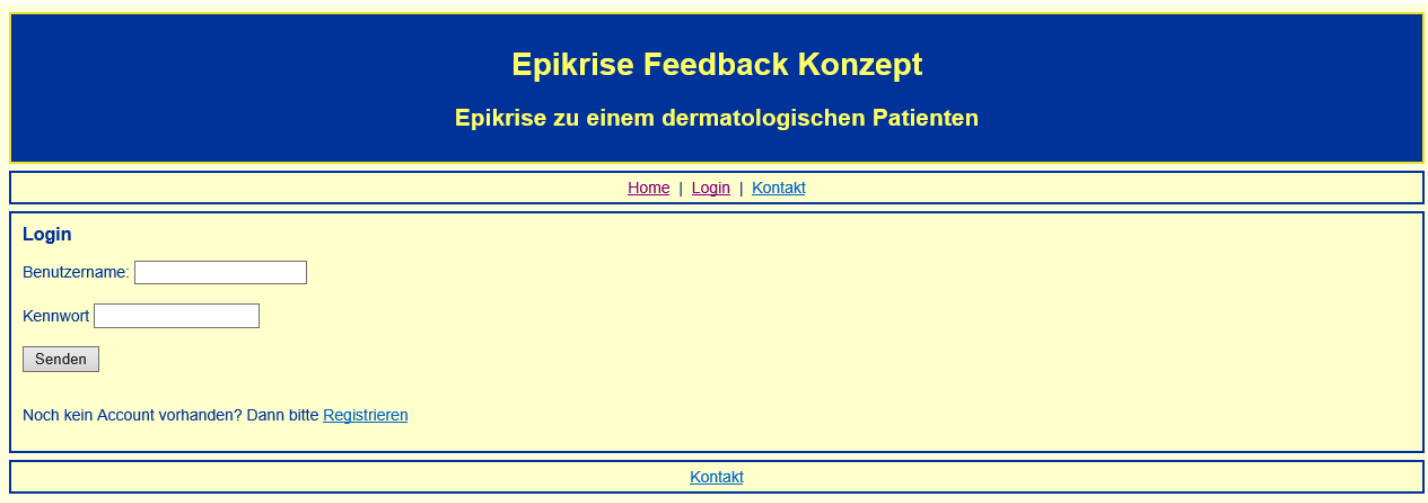

Abbildung 10: Screenshot der Loginseite

Die Studierenden konnten sich mit dem selbst-gewählten Benutzername und Kennwort jederzeit einloggen.

\subsection{Kontakt}

Hier sind die Kontaktdaten (Emailadressen) des Administrators (Doktorand) und von Prof. Ochsendorf angegeben, so dass sich die Studierenden bei Fragen oder Problemen jederzeit melden konnten. Für Fragen hinsichtlich der Webanwendung wurde auf die Emailadresse des Doktoranden verwiesen, für Fragen bzgl. Kurs oder Lerninhalt auf die Emailadresse von Prof. Ochsendorf.

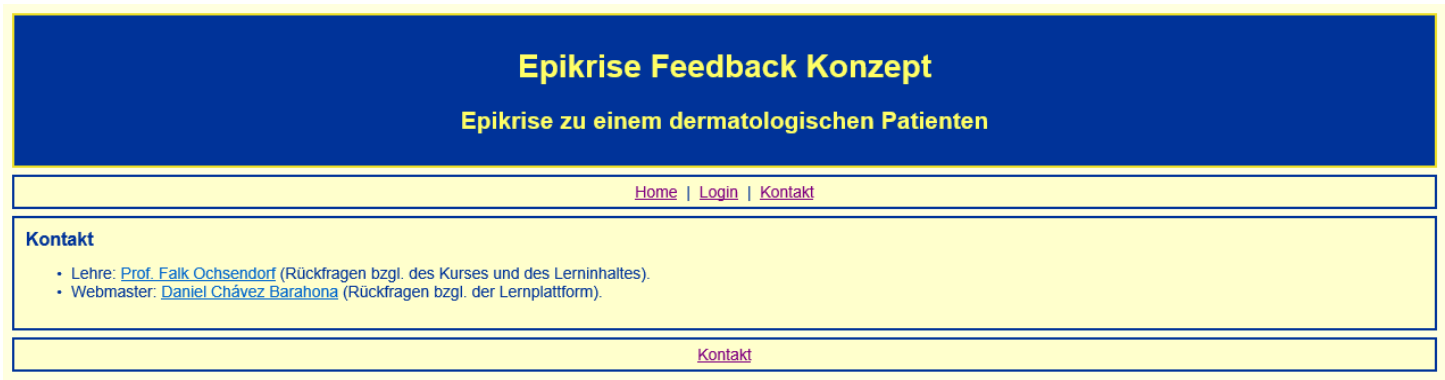

Abbildung 11: Hinterlegte Kontakt-Daten auf der Webanwendung

Sowohl die Emailadressen von Prof. Ochsendorf als auch des Doktoranden waren auf der Webanwendung hinterlegt für den Fall, dass die Studierenden Probleme hätten, Hilfe bräuchten oder Fragen hätten.

\subsection{Hauptmenü}

Das Hauptmenü war nur nach einem erfolgreichen Einloggen sichtbar und beinhaltete folgende Unterseiten:

- Meine Epikrise bearbeiten / anschauen: Auf dieser Seite befindet sich das Formular für die Eintragung und Bearbeitung der eigenen Epikrise. 
- Epikrise 1 korrigieren: Nach Klicken auf den entsprechenden Button gelangte der Studierende zu dem Formular, in welches er die Korrektur der ersten Epikrise eintragen sollte.

- Epikrise 2 korrigieren: Analog befindet sich hier das Formular für die Korrektur der zweiten Epikrise.

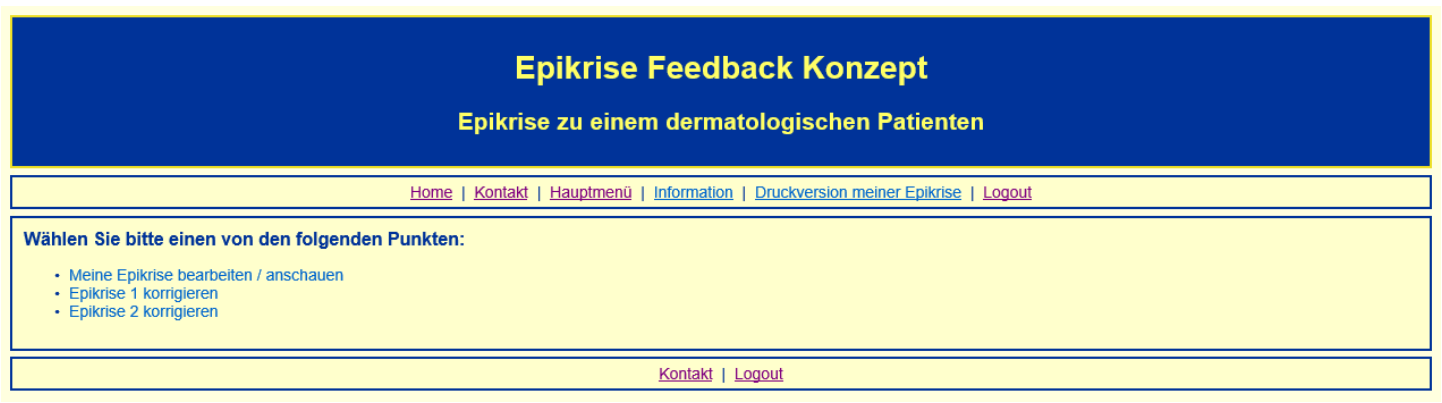

\section{Abbildung 12: Screenshot des Hauptmenüs auf der Webanwendung}

Unter dem Punkt Hauptmenü konnten die Studierenden entweder zu ihrer eigenen Epikrise gelangen oder zu den beiden zu korrigierenden Epikrisen. Dies war jedoch von den jeweiligen Zeiträumen abhängig, wie vorher beschrieben. 
Epikrise Feedback Konzept

Epikrise zu einem dermatologischen Patienten

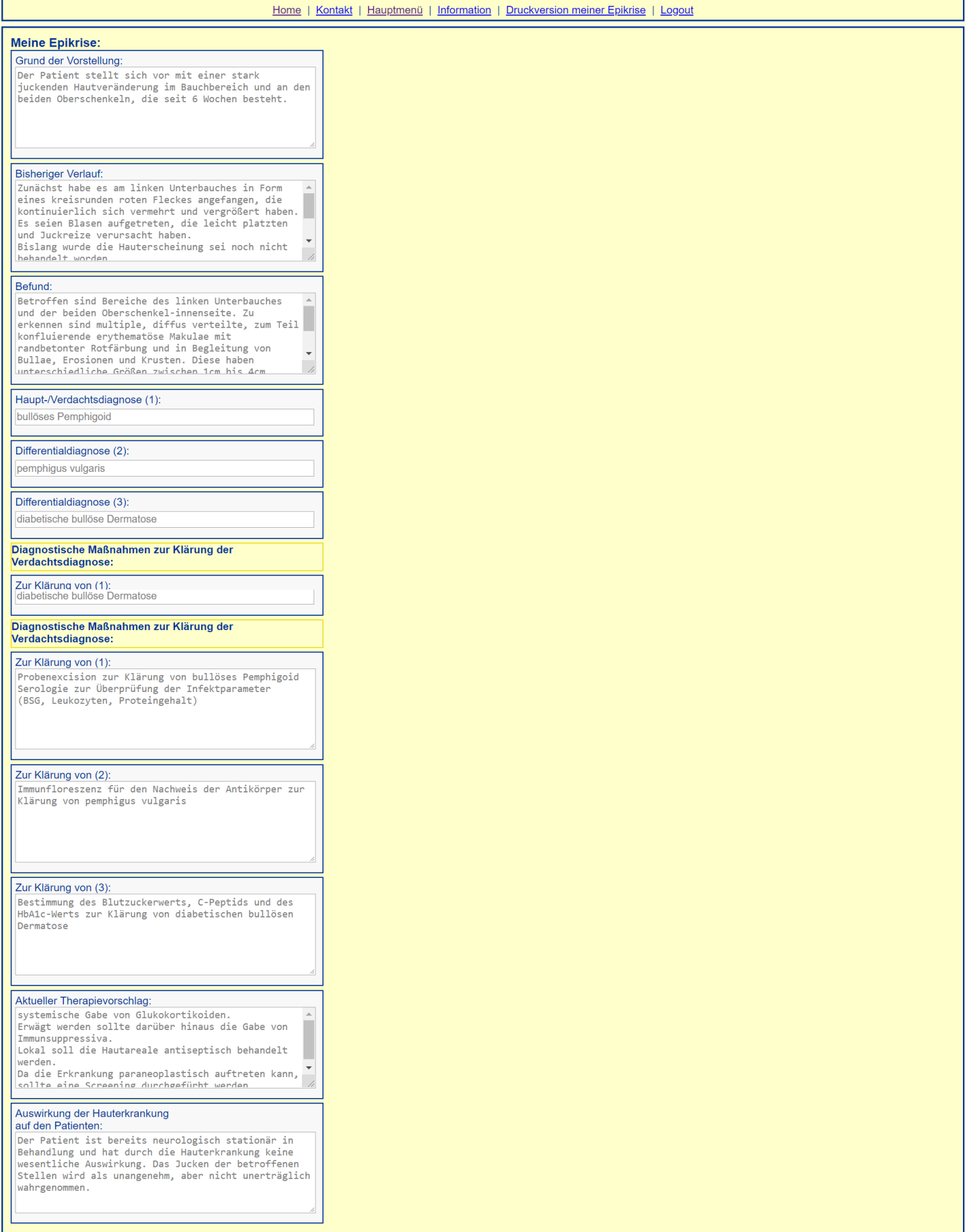

Kontakt | Logout

\section{Abbildung 13: Epikrise-Formular der Webanwendung anhand eines Beispiels}

Man erkennt die einzelnen Unterpunkte, wie Anamnese, Befund, Verdachts- und

Differentialdiagnosen, diagnostische Abklärungen, Therapievorschlag und Auswirkungen auf den Patienten. Die Studierenden konnten einen freien Text, in die dafür zur Verfügung gestellten Textfelder eintragen. 
Epikrise Feedback Konzept

Epikrise zu einem dermatologischen Patienten

Home | Kontakt | Hauptmena | Information | Druckversion meiner Epikrise | Logout

\begin{tabular}{|c|c|}
\hline Epikrise 1 zum Korrigieren: & Korrektur 1: \\
\hline $\begin{array}{l}\text { Grund der Vorstellung: } \\
\text { Rötung und Spannung der Stirn mit Fieber, } \\
\text { schüttel frost und allg. Krankheitsgefüh1. Außerdem } \\
\text { starke Nacken- und Kopfschmerzen. }\end{array}$ & || Kommentar: \\
\hline 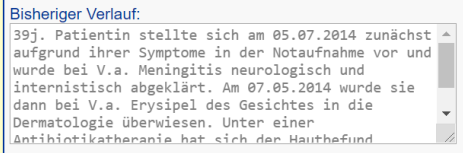 & |Kommentar: \\
\hline 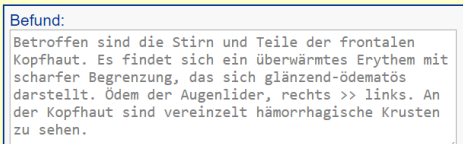 & \begin{tabular}{|l} 
Kommentar: \\
Beschreibung des Hautbefundes bisauf der Formation 5 \\
vollständig.
\end{tabular} \\
\hline $\begin{array}{l}\text { Haupt-Nerdachtsdiagnose (1): } \\
\text { Erysipel }\end{array}$ & Kommentar: \\
\hline \begin{tabular}{|l} 
Differentialdiagnose (2): \\
toxisches Kontaktekzem
\end{tabular} & Kommentar: \\
\hline \begin{tabular}{|l|} 
Differentialdiagnose (3): \\
Meningitis (bei starken Nacken- und Kopfschmerzen)
\end{tabular} & Kommentar: \\
\hline $\begin{array}{l}\text { Diagnostische Maßnahmen zur Klärung der } \\
\text { Verdachtsdiagnose: }\end{array}$ & $\begin{array}{l}\text { Diagnostische Maßnahmen zur Klärung der } \\
\text { Verdachtsdiagnose }\end{array}$ \\
\hline \begin{tabular}{|l|} 
Zur Klärung von (1): \\
Temperatur messen $\rightarrow 39^{\circ} \mathrm{C}$ \\
Entzündungsparameter $\rightarrow$ CRP erhöht und Leukozytose \\
Eintrittspforte identifizieren $\rightarrow>$ Patientin gibt an \\
sich die Kophaut aufgekratzt zu haben bei der Hitze \\
an vergangenen Wochenende
\end{tabular} & Kommentar: \\
\hline \begin{tabular}{|l|} 
Zur Klärung von (2): \\
Anamnestisch nach neuen Salben, Cremes, Schampoos \\
oder Haarfäbemittel fragen, die in Kontakt mit der \\
umschriebenen Läsion gekommen sein können- $\rightarrow$ All dies \\
verneint die Patientin
\end{tabular} & Kommentar: \\
\hline $\begin{array}{l}\text { Zur Klärung von (3): } \\
\text { Lumbalpunktion } \rightarrow \text { O.p.B. } \\
\text { CT } \rightarrow \text { o.p.B. }\end{array}$ & Kommentar: \\
\hline $\begin{array}{l}\text { Aktueller Therapievorschlag: } \\
\text { - Antibiotika-Therapie: } \\
\text { Clindamycin } 60 \theta \mathrm{mg} \mathrm{1-1-1} \\
\text { - Schmerzmedikation } \\
\text { - topisch kühlende Wicke1/ Lotions }\end{array}$ & Kommentar: \\
\hline 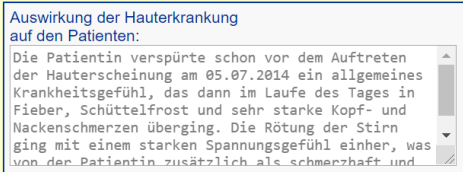 & Kommentar: \\
\hline
\end{tabular}

Kontakt | Logout

\section{Abbildung 14: Screenshot des Korrekturformulars anhand eines Beispiels}

Auf der linken Seite sieht man die zu korrigierende Epikrise. Das Korrekturformular ist so aufgebaut, dass Kommentare zu jedem Punkt direkt daneben geschrieben werden können. 


\subsection{Information}

Beschreibung des Konzepts für das gegenseitige Feedback. Hier sind auch die Fristen / Deadlines beschrieben.

\section{Epikrise Feedback Konzept}

Epikrise zu einem dermatologischen Patienten

Home | Kontakt | Hauptmenu | Information | Druckversion meiner Epikrise | Logout

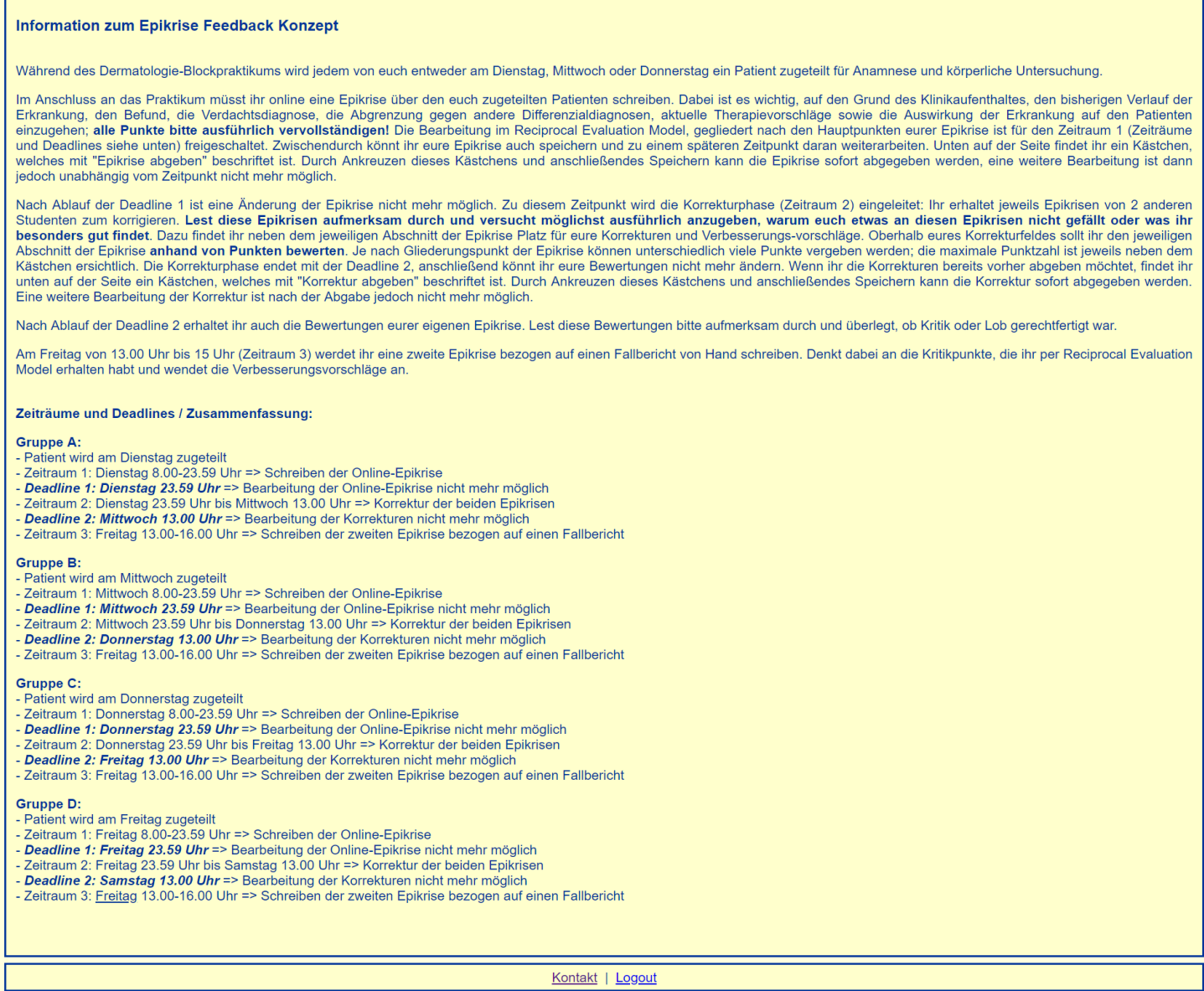

\section{Abbildung 15: Screenshot der Information auf der Webanwendung}

Hier erhalten die Studierenden nochmals eine genaue Anleitung zur Webanwendung sowie eine Übersicht über die Fristen, gegliedert nach den jeweiligen Hospitationsgruppen.

\subsection{Druckversion meiner Epikrise}

Diese Seite erstellte eine Druckversion der eigenen Epikrise. Die

Studierenden mussten die Epikrise an sich nicht drucken, es war lediglich ein Angebot, falls Studierende den Befund gerne ausdrucken wollten. 


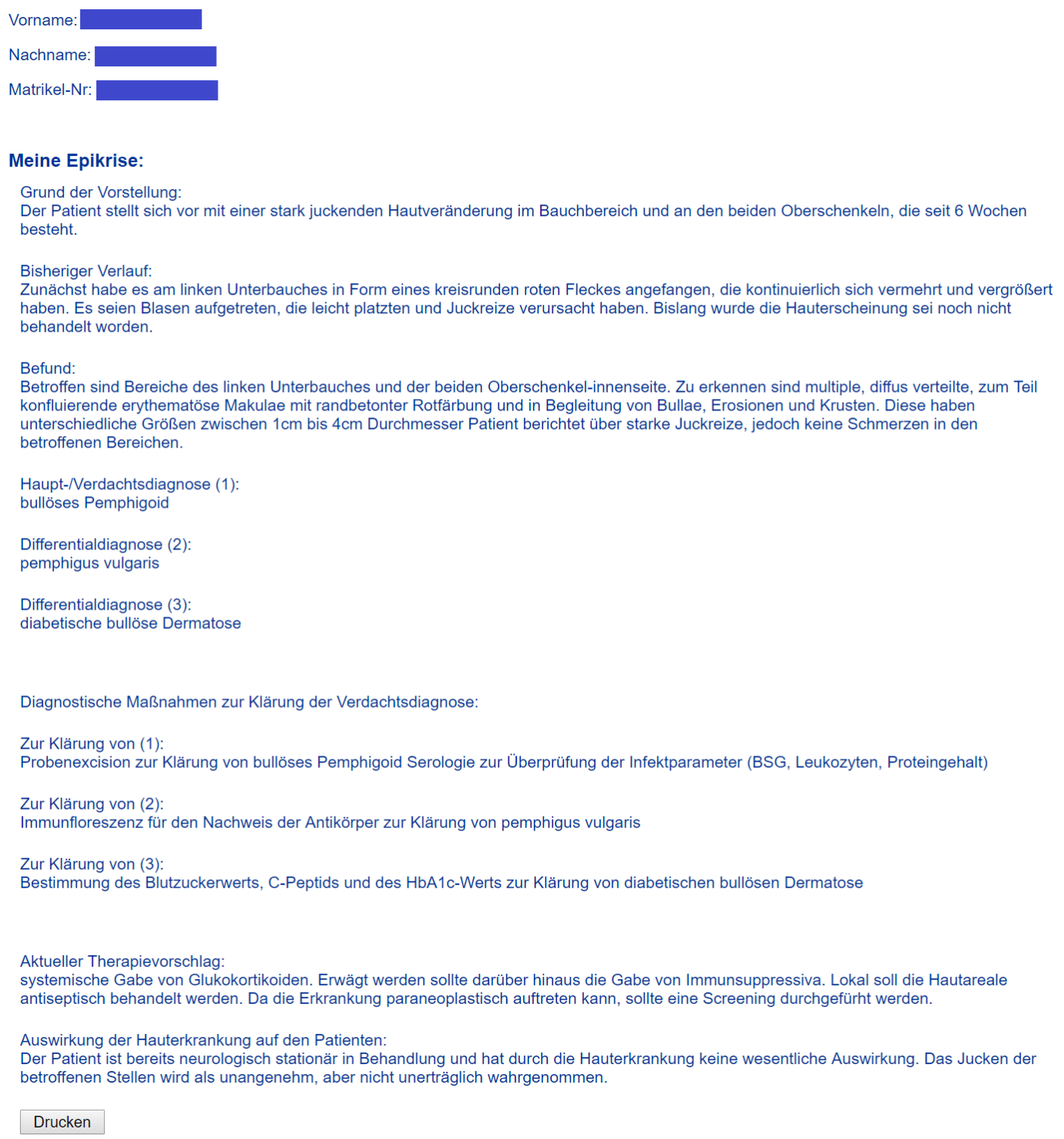

Abbildung 16: Vorschau über die Druckversion der Epikrise anhand eines Beispiels Falls die Studierenden ihre Epikrise drucken wollten, erhielten sie zunächst eine Vorschau der Druckversion. Oben wurde automatisch Vorname, Nachname und Matrikelnummer eingefügt.

\subsection{Logout}

Durch Klicken auf diesen Button konnten die Studierenden die Webanwendung verlassen.

\subsubsection{Sichtbarkeit der Seiten}

Das Hauptmenü, die eigene Epikrise, Information, Druckversion der eigenen

Epikrise und Logout waren nur nach einem erfolgreichen Einloggen in der Webanwendung sichtbar. 
Die Formulare zum Eintragen der Korrekturen waren nur in den jeweiligen Phasen sichtbar (siehe Tabelle 2: Sichtbarkeit der verschiedenen Seiten).

\begin{tabular}{|c|c|}
\hline Seite & Sichtbarkeit \\
\hline Home & Immer \\
\hline Registrierung & Immer \\
\hline Login & Immer \\
\hline Kontakt & Immer \\
\hline Hauptmenü & Nach erfolgreichem Einloggen \\
\hline Information & Nach erfolgreichem Einloggen \\
\hline $\begin{array}{l}\text { Druckversion meiner } \\
\text { Epikrise }\end{array}$ & Nach erfolgreichem Einloggen \\
\hline Epikrise & Nach erfolgreichem Einloggen \\
\hline Korrektur 1 & $\begin{array}{l}\text { Nur in der Phase zur Eintragung der } \\
\text { Korrektur }\end{array}$ \\
\hline Korrektur 2 & $\begin{array}{l}\text { Nur in der Phase zur Eintragung der } \\
\text { Korrektur }\end{array}$ \\
\hline Logout & Nach erfolgreichem Einloggen \\
\hline
\end{tabular}

\section{Tabelle 2: Sichtbarkeit der verschiedenen Seiten}

Während einige Seiten, wie Registrierung, Login, Hauptmenü und Kontakt immer sichtbar waren, gab es andere Seiten, die erst nach erfolgreichem Einloggen sichtbar wurden. Die Korrekturen hingegen konnten nur in bestimmten Zeiträumen aufgerufen werden.

\subsubsection{TESTPHASE}

Für die Testphase wurden Programme in PHP geschrieben, welche eine Simulation der Registrierung, Login, Eintragung der Epikrisen und Korrekturen durchführte.

Diese Testphase simulierte die Anwendung von 1000 Benutzern zum gleichen Zeitpunkt, um sicherzustellen, dass das System nicht 
zusammenbrach, wenn alle Studierenden gleichzeitig auf der Webanwendung aktiv wären.

Zusätzlich wurde eine andere Testphase für die Verteilung der Epikrisen durchgeführt. Die Fristen wurden hierfür verkürzt, um Wartezeit zu vermeiden. Es erfolgten 100 simulierte Durchgänge. Die Ergebnisse wurden anschließend vom Doktoranden manuell überprüft, um die Richtigkeit der Funktionalität zu gewährleisten.

Es erfolgten 3 Testphasen mit 7 verschiedenen Test-Benutzern (Informatiker, Arbeitskollegen des Doktoranden), welche Studierende simulierten und den beschriebenen Ablauf durchführten. Es wurden hierbei keine medizinischen Daten eingetragen, da diese Benutzer keinerlei Verbindung mit der Universität Frankfurt am Main und auch nicht mit dem Medizinstudium hatten. Die Daten, die von dem Test-Benutzer eingetragen wurden, waren somit nur beliebige Texte. Die Datenbank wurde nach alle Testphasen geleert, um Verwirrungen in Bezug auf echte Daten während der Untersuchung zu vermeiden.

\subsubsection{VERFÜGBARKEIT DER DATEN FÜR DIE STUDIERENDEN}

Nach Abschluss der Programmierung und der Testphasen war unsere Webanwendung ab der Kalenderwoche 26 im Jahr 2014 online verfügbar. Einen Monat nach Ende des Semesters wurden die Zugänge der Studierenden deaktiviert

\subsection{BeWERTUNG DURCH DIE STUDIERENDEN}

Nach Abschluss des Kurses erhielten die Studierenden der Experimental- und Kontrollgruppe jeweils einen Evaluationsbogen (siehe Anhang 8: Fragebogen zur Selbsteinschätzung (Kontrollgruppe) und Anhang 9: Fragebogen zur Selbsteinschätzung (Experimentalgruppe)). In der Experimentalgruppe wurden spezifische Fragen zum gegenseitigen Feedback gestellt, zusätzlich jedoch auch allgemeine Fragen zum Kurs. Die allgemeinen Fragen der Experimental- und Kontrollgruppe waren identisch, um einen Vergleich zwischen beiden Gruppen zu ermöglichen. Die Bewertung erfolgte anonym, um eine möglichst ehrliche Antwort der Studierenden zu erhalten. An persönlichen Fragen wurde lediglich nach Alter, Geschlecht, Semester, Anzahl vorhergehender Famulaturen und vorhergehender medizinischer 
Ausbildung gefragt. Bei den Fragen zur Einschätzung gab es eine LikertSkala von 1 (trifft gar nicht zu) bis 6 (trifft voll zu), welche die Studierenden durch Ankreuzen auswählen konnten. Zusätzlich gab es offene Fragen bezüglich Problemen und Verbesserungsvorschlägen, in welche die Studierenden einen freien Text schreiben konnten. Zur Vereinfachung der Auswertung wurden die Kästchen mit 1-6 nummeriert und die durchschnittliche Punktzahl der jeweiligen Gruppen bestimmt. Mit Ausnahme einer Frage nach der Vergabe einer Schulnote von 1-6 war jeweils die 1 die niedrigste mögliche Punktzahl, während die 6 die höchstmögliche Punktzahl war.

\subsection{Statistische Auswertung}

Die Null-Hypothese war, dass es keinen Unterschied zwischen Kontroll- und Experimentalgruppe gibt, hinsichtlich der erreichten Punktzahl in der TEDKlausur (t-Test, Signifikanzniveau $p<0,05$ ).

Die weiteren Berechnungen waren rein deskriptiv (Prismversion 6.00 for Windows, GraphPad Software, La Jolla California USA, www.graphpad.com". GraphPadPrism).

\subsection{ETHIKVOTUM}

Im Vorfeld der Untersuchung wurde bei der Ethik-Kommission des Fachbereichs Medizin anhand einer Projekt-Skizze nach der Notwendigkeit eines entsprechenden Ethik-Antrags gefragt (Schreiben vom 31.05.2013). Dies wurde nicht für nötig erachtet.

\section{ERGEBNISSE}

\subsection{Elektronische UmSetZung des Gegenseitigen FeedBacks}

Es wurden keine Fehlermeldungen vom System registriert; es kam weder zu einem Absturz des Servers noch zu Übertragungsproblemen während des Einsatzes der Webanwendung. Die Daten wurden von dem Server richtig und rechtzeitig auf den Sicherheitsserver übertragen. Hierbei erfolgte die Übertragung sämtlicher Informationen immer 1 zu 1 mit derselben Anzahl von 
Dateien und Einträgen. Dies wurde in den Logdateien des Systems täglich überprüft.

Die lokale Sicherheitskopie auf den eigenen Rechnern des Doktoranden ist ebenfalls einwandfrei im Stundentakt gelaufen.

Alle Informationen wurden ohne Fehler und problemlos eingetragen und in der Datenbank gespeichert. Die Daten der Datenbank wurden in Tabellenkalkulationsprogramme importiert und konnten somit für die vorliegende Arbeit verwendet werden.

Die Verteilung der Epikrisen lief einwandfrei. In den entsprechenden Zeiträumen wurde die Ansicht für die Studierenden immer korrekt dargestellt bezüglich Anzeige einer fremden Epikrise oder Anzeige der Korrekturen. Auch von Seiten der Studierenden wurden während des DermatologiePraktikums keine Probleme gemeldet bzgl. Fehlern, Darstellungsproblemen oder Ausfällen des Servers.

Die Testphase vor dem Einsatz der Webanwendung lief ebenfalls einwandfrei. Sowohl die manuellen Tests durch die Informatiker als auch die im Hintergrund laufenden Servertests (siehe 2.6.5 Testphase) liefen ohne Fehlermeldungen. Während jeder manuellen Testphase schalteten wir 3 Mal den Hauptserver aus; die Test-Benutzer merkten keine Änderung in der Webanwendung und wussten nicht, dass der Hauptserver während der Tests ausgeschaltet worden war. Der Sicherheitsserver übernahm bei diesen Tests seine Funktion einwandfrei. Der Ausfall der Hauptserver wurde bei dem Test in den Logdateien von beiden Servern dokumentiert und Warnungsemails wurden an den Administrator versendet.

\subsection{TEILNAHME AN DER ABSCHLUSSKLAUSUR (TED-KLAUSUR)}

Es haben 159 Studierende von insgesamt 181 (88\%) an der TED-Klausur teilgenommen, in der Kontrollgruppe 59 von 76 Studierenden (78\%), in der Experimentalgruppe 100 von 105 Studierenden (95\%), wie man in der Abbildung 17 erkennen kann. Gründe für die Nicht-Teilnahme an der TEDKlausur waren Verschieben auf das nächste Semester sowie krankheitsbedingte Ausfälle. Kein Studierender brach den Kurs wegen der hier dargestellten Untersuchung ab. 5 Studierende der Experimentalgruppe meldeten sich für die Webanwendung an und schrieben die erste Epikrise 
online, nahmen jedoch nicht an der TED-Klausur teil und wurden somit nicht in die Studie eingeschlossen.

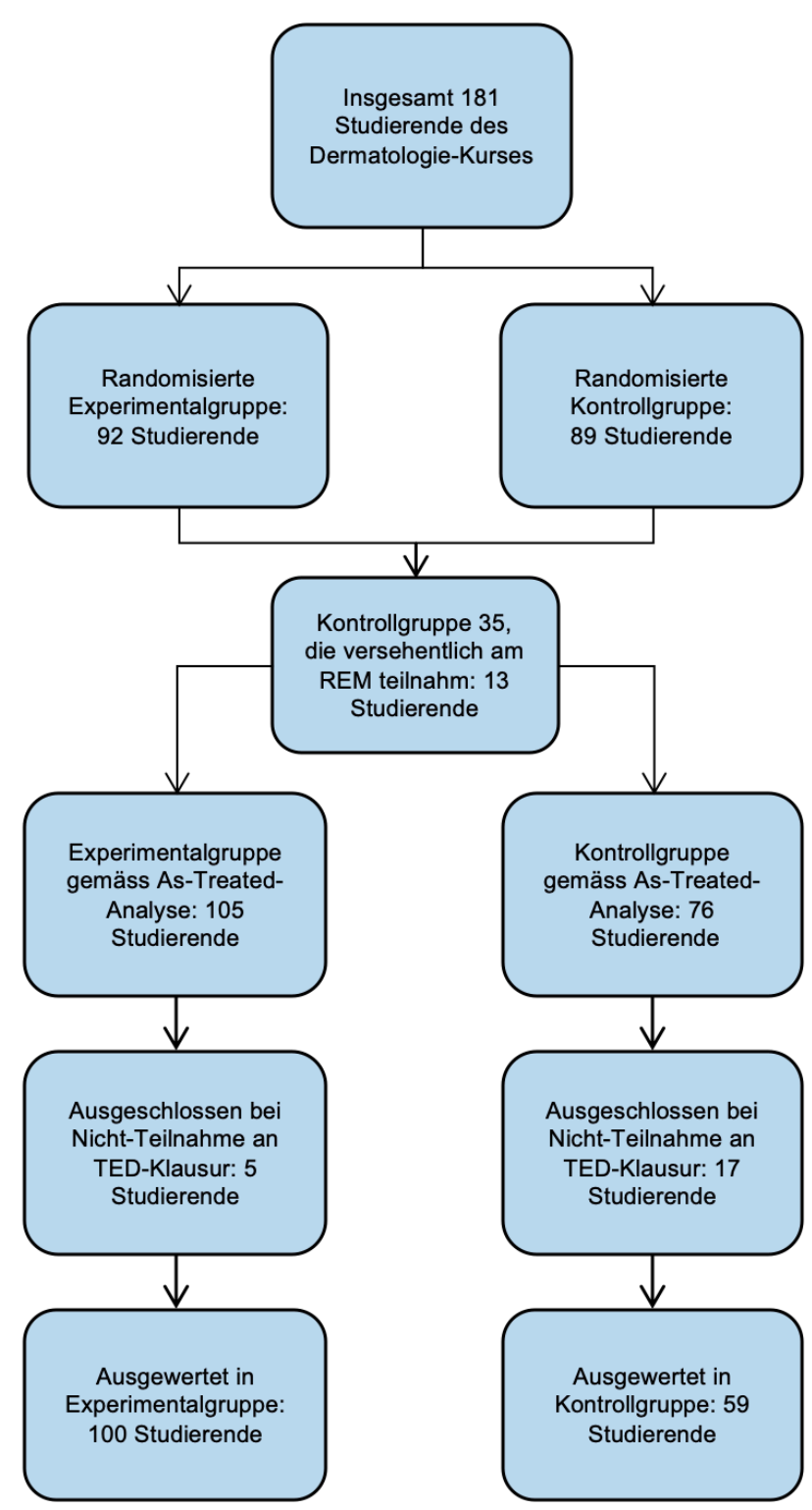

Abbildung 17: Randomisierung und Teilnahme der Studierenden an der TED-Klausur In der Experimentalgruppe wurden 5 Studierende ausgeschlossen aufgrund Nichtteilnahme an der TED-Klausur, in der Kontrollgruppe waren es 17 Studierende. Somit wurden in die Auswertung 100 Studierende aus der Experimentalgruppe und 59 Studierende aus der Kontrollgruppe mit einbezogen.

\subsection{BestehensquOTE}

Die Bestehensgrenze lag bei 10 Punkten von maximal erreichbaren 20

Punkten in der TED-Klausur. Insgesamt hatten 6 Studierende von 59 aus der 
Kontrollgruppe $(10,17 \%)$ und 5 Studierende von 100 aus der Experimentalgruppe (5\%) die TED-Klausur beim ersten Versuch nicht bestanden. Somit war die Nicht-Bestehensquote der Kontrollgruppe doppelt so hoch wie die der Experimentalgruppe. Dieser Unterschied war jedoch nicht signifikant ( $p$-Wert 0,39$)$.

\section{Bestehensquote}

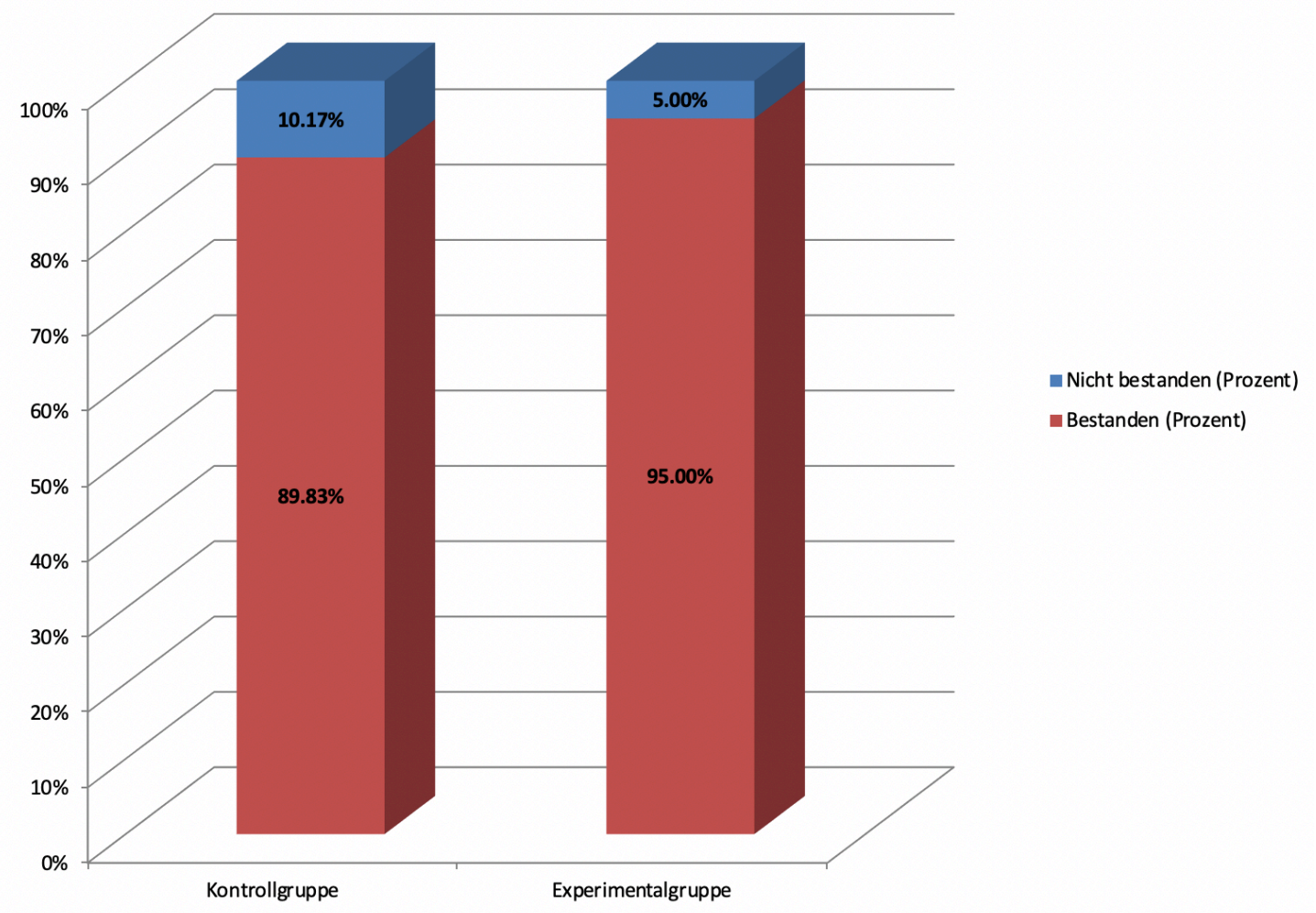

Abbildung 18: Bestehensquote in Experimental- und Kontrollgruppe

In der Kontrollgruppe hatten 6 von 59 Studierenden (10,17\%) die erste Klausur nicht bestanden, in der Experimentalgruppe 5 von 100 Studierenden (5\%). Es zeigte sich somit kein signifikanter Unterschied hinsichtlich der Bestehensquote.

\subsection{VERGLEICH EXPERIMENTALGRUPPE UND KONTROLLGRUPPE}

Die Studierenden der Kontrollgruppe erreichten durchschnittlich 12,08 Punkte in der TED-Klausur, während die Studierenden der Experimentalgruppe durchschnittlich 13,16 Punkte erreichten. Es zeigte sich somit in der Experimentalgruppe eine signifikante Verbesserung von etwa einem Punkt (= $5,44 \% ; p=0,0042$ ) in Bezug auf die maximal erreichbare Punktzahl von 20 Punkten. 


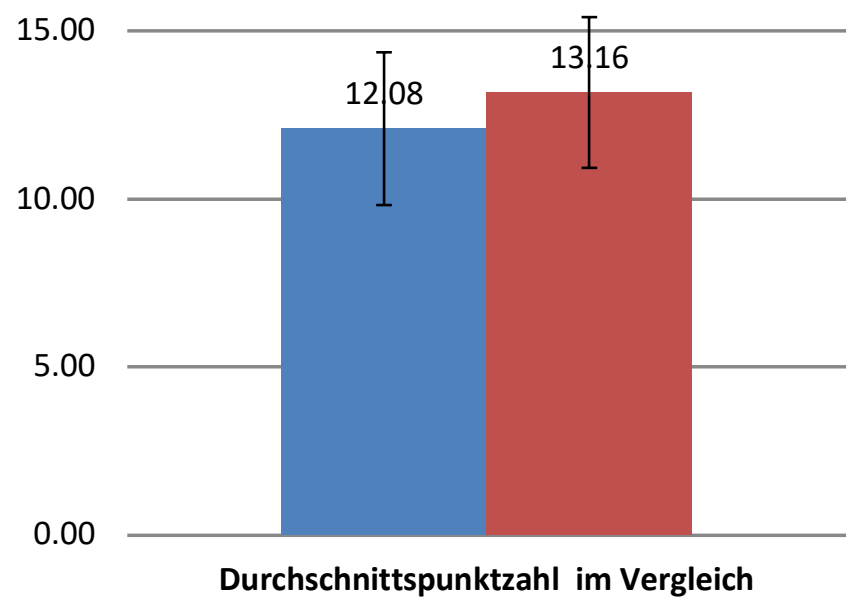

Durchschnittspunktzahl Kontrollgruppe

- Durchschnittspunktzahl Experimentalgruppe

\section{Abbildung 19: Vergleich der Punktzahl zwischen Kontroll- und Experimentalgruppe}

Die Studierenden der Kontrollgruppe hatten durchschnittlich 12,08 Punkte erreicht in der TED-Klausur, während die Studierenden der Experimentalgruppe durchschnittlich 13,16 Punkte erreichten $(p=0,0042)$.

\subsection{TEILNAHMEBEREITSCHAFT AM GEGENSEITIGEN FEEDBACK}

Die Teilnahmebereitschaft war unter den Studierenden trotz Bemühung um Motivation und trotz zahlreicher Mitteilungen leider nicht optimal. Von den 100 Studierenden der Experimentalgruppe, welche die TED-Klausur schrieben, hatten sich nur 75 überhaupt für die Webanwendung für das gegenseitige Feedback angemeldet. Von diesen 75 Studierenden hatten nur 60 Studierende die erste Epikrise geschrieben. Von diesen 60 Studierenden wiederum hatten nur 34 Studierende eine Korrektur für eine fremde Epikrise geschrieben. Von allen 100 Studierenden hatte keiner 2 Korrekturen geschrieben. Somit hatte kein einziger Studierender das gegenseitige Feedback korrekt durchgeführt (siehe Abbildung 20). 


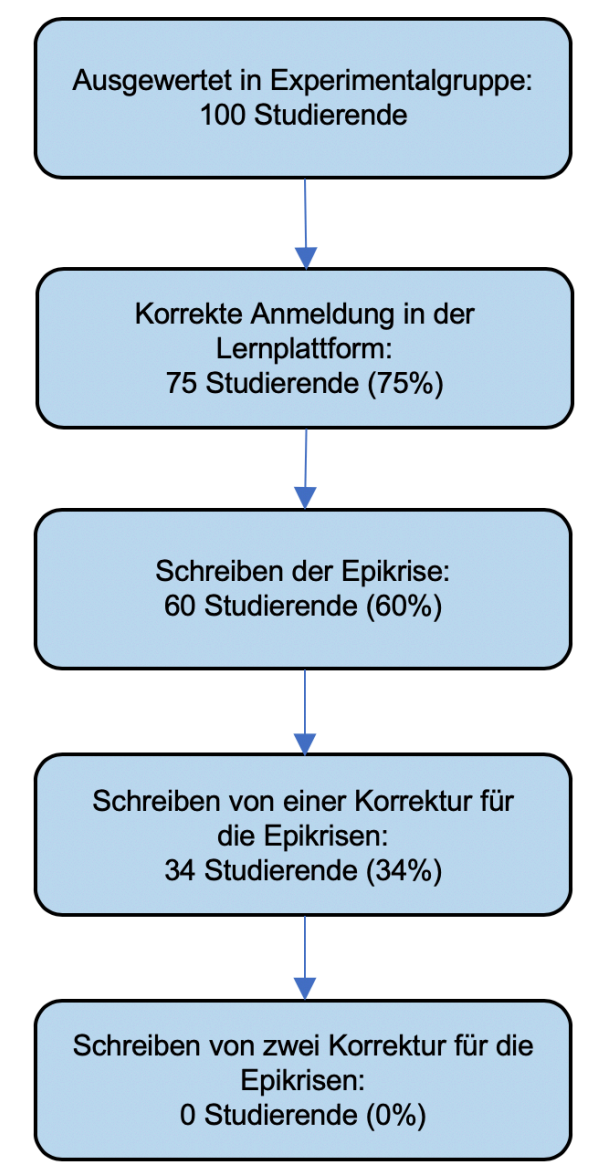

\section{Abbildung 20: Teilnahme am gegenseitigen Feedback}

Von den 100 Studierenden in der Experimentalgruppe meldeten sich 75 für die Webanwendung an. Nur 60 Studierende schrieben eine Epikrise und nur 34 schrieben eine Epikrise und eine Korrektur. Insgesamt hat kein Studierender der Experimentalgruppe korrekt am gegenseitigen Feedback teilgenommen und wie gewünscht 2 Korrekturen geschrieben.

\subsection{Punkte bei der Teilnahme am gegenseitigen Feedback}

Die durchschnittlich erreichte Punktzahl in der TED-Klausur bei den Studierenden der Experimentalgruppe lag bei 13,16/20 Punkten. Bei genauerer Analyse hinsichtlich der korrekten Teilnahme zeigte sich, dass diejenigen, welche nur die Online-Epikrise geschrieben hatten ohne fremde Epikrisen zu korrigieren, durchschnittlich 13,52 Punkte erreichten $(p=0,34)$. Die Studierenden der Experimentalgruppe, welche die Online-Epikrise geschrieben hatten und zusätzlich eine Korrektur geschrieben hatten, erreichten durchschnittlich 13,44 Punkte $(p=0,54)$. 


\section{Durchschnittspunktzahl der Experimentalgruppe im Vergleich}

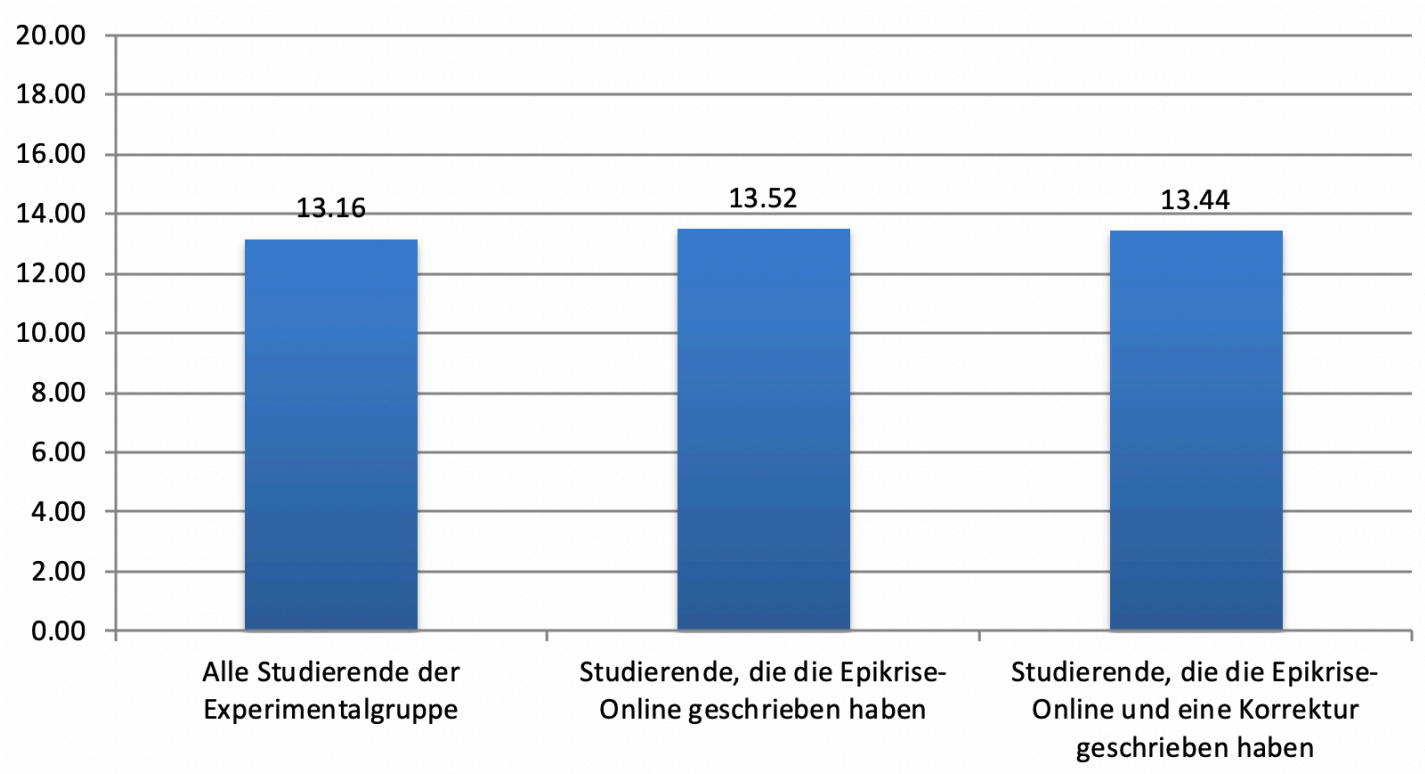

Abbildung 21: Durchschnittlich erreichte Punktzahl der Experimentalgruppe

Es erfolgte eine getrennte Auswertung hinsichtlich der korrekten Teilnahme am gegenseitigen Feedback. Somit wurden Studierende, welche die Online-Epikrise geschrieben haben und Studierende, welche die Online-Epikrise und eine Korrektur geschrieben haben, getrennt ausgewertet. Dabei zeigte sich jedoch kein signifikanter Unterschied hinsichtlich der Punktzahl in Abhängigkeit der korrekten Teilnahme am gegenseitigen Feedback.

\subsection{BEWERTUNG DES «ABSCHLUSSFALLS»}

Der Abschlussfall wurde bei allen Studierenden mit denselben Kriterien (siehe 2 Material und Methoden) und von derselben Person (Doktorand) ausgewertet. Die maximal erreichbare Punktzahl lag bei 14 Punkten. Die Studierenden der Kontrollgruppe erreichten durchschnittlich 8,32 Punkte, während die Studierenden der Experimentalgruppe durchschnittlich 8,98 Punkte erreichten. Es zeigte sich ein Unterschied von 0,66 Punkten $(=4,72$ $\% ; p=0,043)$

\subsubsection{ERREICHTE PUNKTZAHL BEI DER BEFUNDBESCHREIBUNG}

Bei der Befundbeschreibung hatten die Studierenden der Experimentalgruppe ebenfalls besser als die Kontrollgruppe abgeschnitten. Die maximal erreichbare Punktzahl bei der Befundbeschreibung betrug 5 Punkte. Die Experimentalgruppe erreichte eine durchschnittliche Punktzahl von 3,71 
Punkten während die Kontrollgruppe 3,40 Punkte erreichte. Es zeigte sich somit eine Verbesserung der Punktzahl bei der Befundbeschreibung von 0,31 Punkten $(6,19 \% ; p=0,033)$.

\subsection{BeWERTUNG DES EPIKRISE FEEDBACK SYSTEMS DURCH DIE STUDIERENDEN}

Die Punktzahlen der Fragen aus Kontroll- und Experimentalgruppe sind in der Tabelle 3 zusammengefasst. Die ersten Fragen bezüglich Alter, Geschlecht, klinischem Semester, Anzahl der abgeschlossenen medizinischen Famulaturen und vorherigen medizinischen Ausbildungen, wurden für die Bewertung nicht berücksichtigt. Alle Punkte beziehen sich auf eine Skala von 1 bis 6 , wobei 6 die beste Bewertung, 1 die schlechteste Bewertung ist. Die einzige Ausnahme ist hier die Frage nach Bewertung des gegenseitigen Feedbacks mit einer Schulnote von 1 bis 6; hier wurde das deutsche Schulsystem verwendet, wo 1 für «sehr gut» steht, während 6 für «ungenügend» steht.

\begin{tabular}{|l|c|c|}
\hline & $\begin{array}{c}\text { Durchschnittliche } \\
\text { Punktzahl der } \\
\text { Kontrollgruppe }\end{array}$ & $\begin{array}{c}\text { Durchschnittliche } \\
\text { Punktzahl der } \\
\text { Experimentalgruppe }\end{array}$ \\
\hline $\begin{array}{l}\text { Bereits vor dem Kurs habe ich mir } \\
\text { zugetraut Befunde zu beurteilen. }\end{array}$ & 2,11 & 2,90 \\
\hline $\begin{array}{l}\text { Der Kurs hat dazu beigetragen, dass ich } \\
\text { Befunde besser beschreiben kann. }\end{array}$ & 4,83 & 4,49 \\
\hline Differenz & 2,72 & 1,60 \\
\hline $\begin{array}{l}\text { Wie beurteilen Sie folgende Ansätze, Ihre } \\
\text { Fähigkeit zur Befundbeschreibung zu } \\
\text { verbessern: }\end{array}$ & - & - \\
\hline Seminar & & 4,86 \\
\hline Hausaufgabe & 3,92 & 4,46 \\
\hline Virtuelle Poliklinik & 4,66 & 3,60 \\
\hline Hospitation & 3,97 & \\
\hline
\end{tabular}




\begin{tabular}{|l|c|c|}
\hline \multicolumn{1}{|c|}{ Schreiben der Epikrise } & 3,92 & 4,05 \\
\hline \multicolumn{1}{|c|}{ Bed-Side-Teaching } & 4,75 & 4,27 \\
\hline \multicolumn{1}{|c|}{ Feedback } & - & 3,36 \\
\hline $\begin{array}{l}\text { Meine Motivation an dem gegenseitigen } \\
\text { Feedback teilzunehmen war hoch. }\end{array}$ & - & 3,51 \\
\hline $\begin{array}{l}\text { Durch das gegenseitige Feedback fühle } \\
\text { ich mich sicherer bei der Beschreibung } \\
\text { von Befunden. }\end{array}$ & - & 3,11 \\
\hline $\begin{array}{l}\text { Das Feedback hat mich motiviert, mehr zu } \\
\text { lernen. }\end{array}$ & - & 3,19 \\
\hline $\begin{array}{l}\text { Durch das Feedback hatte ich einen } \\
\text { verstärkten Wissenszuwachs. }\end{array}$ & - & 3,26 \\
\hline $\begin{array}{l}\text { Das Konzept des gegenseitigen Feedbacks } \\
\text { war verständlich. }\end{array}$ & - & 4,03 \\
\hline $\begin{array}{l}\text { Die Benutzung der Lernplattform war } \\
\text { verständlich. }\end{array}$ & - & 4,67 \\
\hline $\begin{array}{l}\text { Ich gebe dem Ansatz folgende Schulnote: } \\
\text { 1=sehr gut, 6 = ungenügend) }\end{array}$ & - & \\
\hline & - & \\
\hline
\end{tabular}

Tabelle 3: Durchschnittliche Punktzahl der Fragen zur Selbsteinschätzung in Experimental- und Kontrollgruppe

In der Experimentalgruppe wurden zusätzliche Fragen zum gegenseitigen Feedback und der Anwendung der Lernplattform gestellt. 


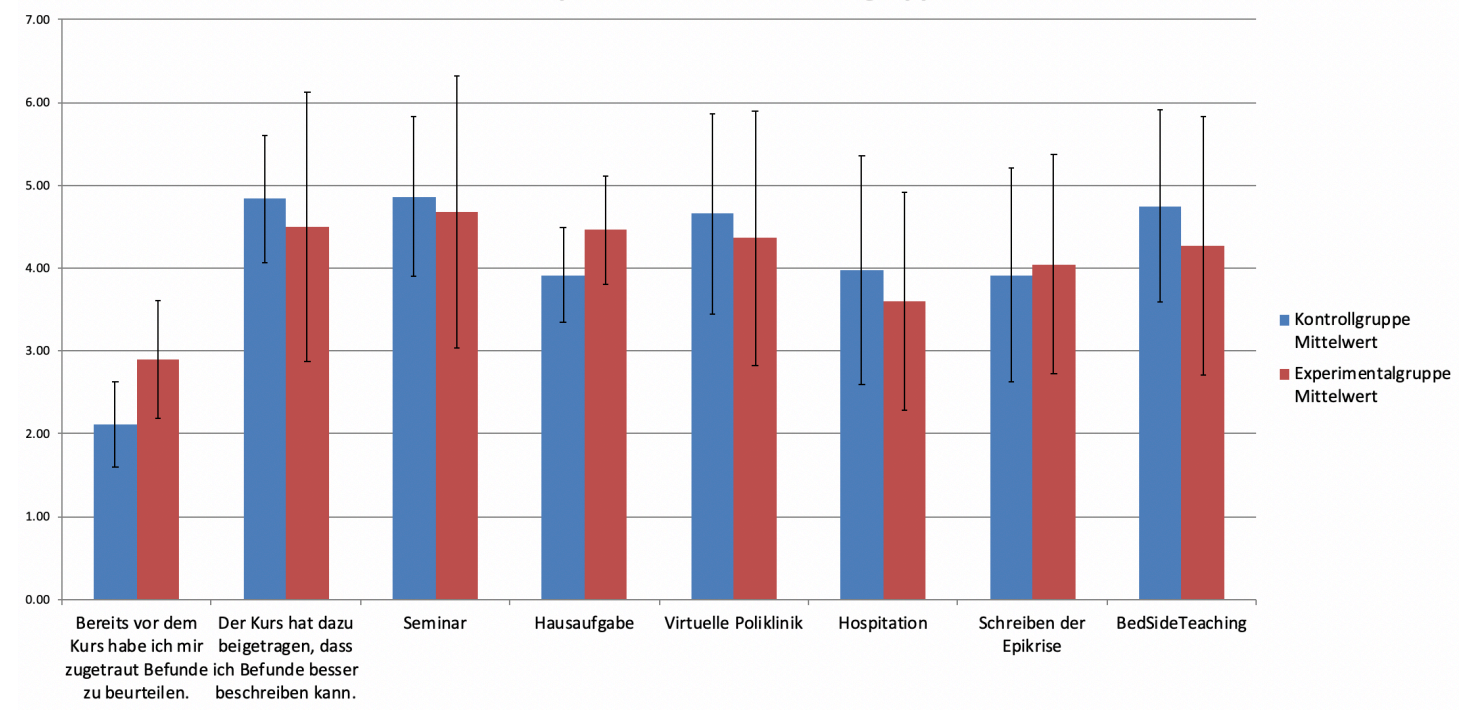

Abbildung 22: Vergleich der durchschnittlichen Punktzahl der Fragen zur

Selbsteinschätzung in Experimental- und Kontrollgruppe

In der Experimentalgruppe hatten sich die Studierenden vor dem Kurs signifikant besser eingeschätzt, verglichen mit der Kontrollgruppe. Hierbei zeigt sich, dass sich die meisten Studierenden eher schlecht eingeschätzt hatten, während sich nur wenige gut eingeschätzt hatten. Den Wissenszuwachs durch Kurs, Seminar, virtuelle Poliklinik, Hospitation, Schreiben der Epikrise und Bed Side Teaching schätzten die Studierenden in beiden Gruppen ähnlich ein. Lediglich den Wissenszuwachs durch Hausaufgaben schätzten die Studierenden der Experimentalgruppe signifikant höher ein verglichen mit der Kontrollgruppe.

\subsubsection{SELBST-EINSCHÄTZUNG DER VERBESSERUNG DURCH DIE STUDIERENDEN}

Zur Auswertung der Selbst-Einschätzung, wurden in beiden Gruppen die Punktzahlen von Frage 6 und 7 (Kontrollgruppe, siehe Anhang 8 bzw. Frage 14 und 15 (Experimentalgruppe, siehe Anhang 9) betrachtet, also der Frage nach Selbsteinschätzung der Befundbeurteilung vor dem Kurs und die Verbesserung der Befundbeschreibung nach dem Kurs.

Hierbei zeigte sich vor dem Kurs in der Experimentalgruppe eine Selbsteinschätzung von 2,90 während diese in der Kontrollgruppe bei 2,11 lag $(p=0,003)$.

Auf die möglichen Gründe dieses signifikanten Unterschieds wird in der

Diskussion eingegangen (siehe 4.3.4.1 Selbsteinschätzung durch die Studierenden). 
Hinsichtlich der Verbesserung der Befundbeschreibung durch den Kurs zeigte sich kein Unterschied (4,49 Punkte in der Experimentalgruppe, 4,83 Punkte in der Kontrollgruppe; $p=0,23$ ).

Die Einschätzung der Verbesserung durch die jeweiligen Unterkategorien Seminar, virtuelle Poliklinik, Hospitation, Schreiben der Epikrise und Bed-Side Teaching zeigte in beiden Gruppen ähnliche Zahlen ohne signifikante Unterschiede. Lediglich in der Unterkategorie Hausaufgabe vergaben die Studierenden der Experimentalgruppe durchschnittlich 4,46 Punkte, während die Studierenden der Kontrollgruppe 3,92 Punkte vergaben $(p=0,03)$.

\subsubsection{BEWERTUNG DES GEGENSEITIGEN FEEDBACKS DURCH DIE}

\section{STUDIERENDEN}

Bei der Frage nach der Einschätzung des Anteils des gegenseitigen Feedbacks an der Verbesserung (Experimentalgruppe Frage Nr. 8) wurden durch die Studierenden der Experimentalgruppe durchschnittlich 3,36 Punkte vergeben, somit etwas weniger als für die anderen Unterkategorien (Seminar, Hausaufgaben, virtuelle Poliklinik, Hospitation, Schreiben der Epikrise, Bedside-Teaching).

Gemäß Selbsteinschätzung hat die Frage nach der Motivation für die Teilnahme am Feedback-Modell im Fragebogen durchschnittlich 3,51 Punkte ergeben und liegt somit knapp in der Hälfte zwischen den Antworten „Trifft überhaupt nicht zu“ und „Trifft vollkommen zu“.

Das Feedback-Konzept wurde als verständlich bewertet (4,08 Punkte), die Benutzung der der Lernplattform mit 4,67 Punkten.

Der Ansatz wurde mit einer durchschnittlichen Schulnote (deutsches Schulsystem) von 2,93 bewertet.

Die Frage nach Zunahme der Sicherheit in der Befundbeschreibung durch das gegenseitige Feedback, die Motivation dadurch mehr zu lernen und nach dem Wissenszuwachs durch Feedback wurden nur durchschnittlich bewertet (3,51 und 3,11 Punkte respektiv). 


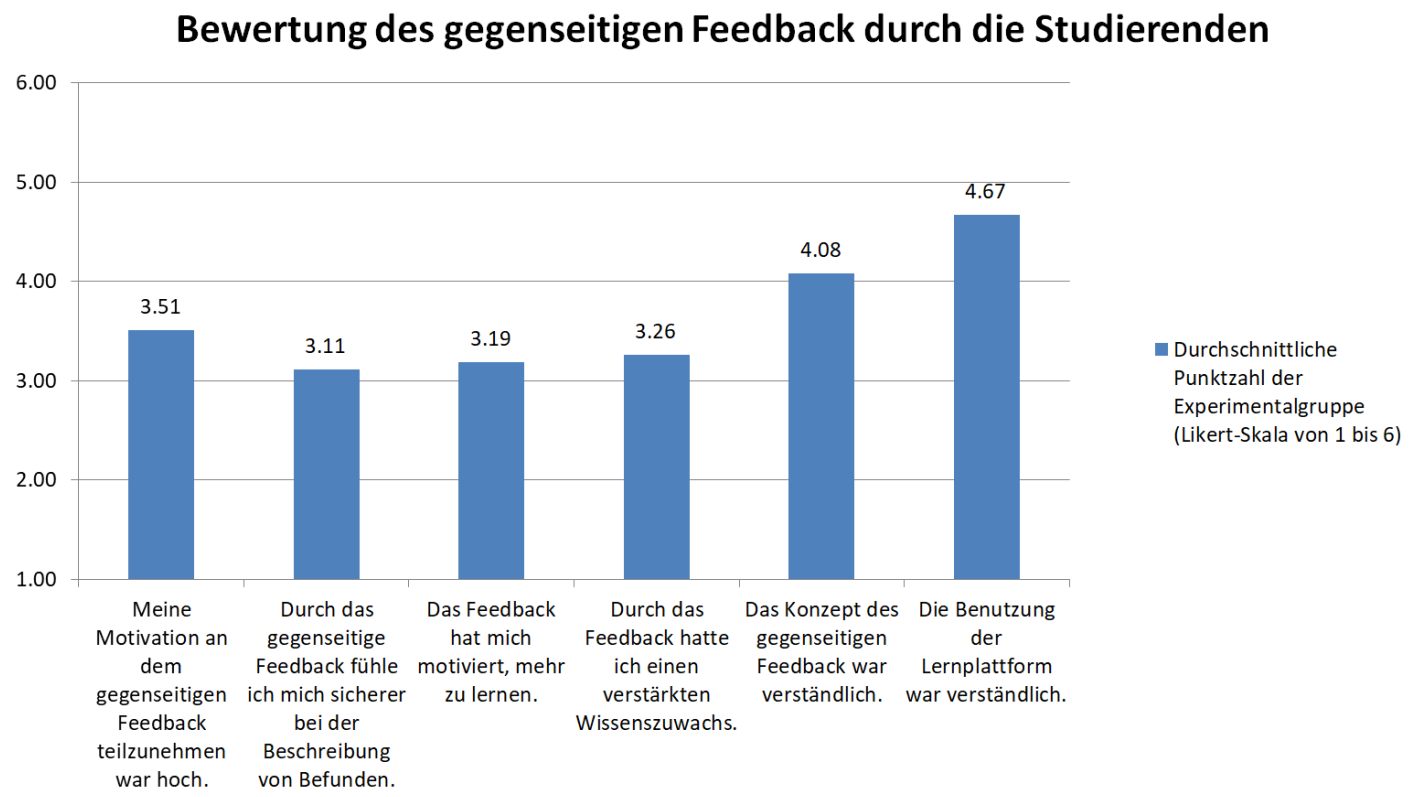

Abbildung 23: Bewertung des gegenseitigen Feedbacks durch die Studierenden

In einem Fragebogen wurde die Experimentalgruppe bei Abschluss des Praktikums auch zur Einschätzung hinsichtlich des gegenseitigen Feedbacks gefragt. Sie konnten hierbei Punkte von 1-6 nach der Likert-Skala vergeben (1 als niedrigste Punktzahl, 6 als höchste Punktzahl). Hierbei ergaben sich vor allem für die Verständlichkeit des Konzepts als auch für die Handhabung der Lernplattform gute Bewertungen.

In den freien Kommentaren der Fragebögen äußerten viele Studierenden als Kritikpunkt, dass sie keine Korrektur oder keine Epikrise zum Korrigieren erhalten haben. Ein weiterer Kritikpunkt war für viele Studierende auch der Umgang mit den Fristen; viele Studierenden hatten den Wunsch nach einer Verlängerung der Fristen. Die meisten Studierenden äußerten sich aber dennoch positiv über das Prinzip des Feedback Models, insbesondere da sie eine Beurteilung durch andere Studierende mit ähnlichem Wissensstand mehr schätzen als eine Beurteilung durch Professoren oder Dozenten. Auch die Anonymität gaben viele Studierende als positiv an sowie die Tatsache, dass sie sich mehr Mühe für die eigene Epikrise gaben, da sie wussten, dass andere Studierende sie korrigieren würden. 


\section{DISKUSSION}

\subsection{Allgemeines}

Das Dermatologie-Praktikum wurde über viele Jahre entwickelt. Ein wesentliches Ziel war es, die Fähigkeit zu vermitteln, Hautbefunde adäquat zu beschreiben. Diese Befundbeschreibung zu erlernen ist erforderlich, um Hautveränderungen adäquat festzuhalten, sich mit Kollegen austauschen zu können bzw. dermatologischen Rat einzuholen und vor allem, um selbst genau hinzusehen und zu verstehen, welche pathophysiologischen Vorgänge in der Haut vorgehen. Deshalb waren Befundbeschreibungen immer integraler Teil des Kurses. So wurden diese bei den «Hausaufgaben» im Kurs, während der interaktiven Seminare sowie während des Bed-SideTeachings wiederholt durchgeführt und reflektiert. Die Befundbeschreibungen der Patienten der «Virtuellen Poliklinik» hatten Musterlösungen, mit denen die Studierenden ihre Angaben vergleichen konnten. Jedoch erhielten die Studierenden bisher kein Feedback über die zwei Befundbeschreibungen, die sie bei den untersuchten Patienten schriftlich angefertigt hatten. Dies lag zum einen daran, dass diese Beschreibungen trotz Ermahnungen oft erst verzögert beim Tutor abgegeben wurden, oft erst am letzten Kurstag, zum anderen an der fehlenden Zeit, diese in Ruhe mit den Studierenden zu besprechen. Deshalb sollte eine Lösung gefunden werden, welche ein Feedback für diese Aktivität ermöglicht.

\section{Feedback}

Nach Hattie und Timperley (2007) ist Feedback definiert als eine Information, die von einer Person (z. B. Lehrer, Gleichaltriger / Peer, Elternteil) zu Aspekten der eigenen Leistung oder seines Verständnisses bereitgestellt wird. Ein Lehrer oder Elternteil kann den Lernenden korrigieren und ermutigen, Gleichaltrige / Peers können eine alternative Strategie vorschlagen. Dieser Abgleich der eigenen Leistung mit einer optimalen Lösung kann auch durch Selbstreflexion, wie Nachschlagen in einem Buch, erfolgen, indem der Lernende dort selbst nach Lösungen sucht und die Richtigkeit seiner Handlung, Aussage oder Antwort bewertet [8, 9]. 
Feedback ist also ein essentieller Bestandteil des effektiven Lernens. Es hilft Lernenden, in diesem Fall Studierenden, eigene Wissensdefizite zu erkennen und leitet an, wie das eigene Lernen verbessert werden kann. In traditionellen Lernformen, wie dem Lehrlingsmodell, und in neuen Ansätzen, wie dem Lernen durch aktives Üben, bringt der Meister dem Lernenden sein Handwerk bei und korrigiert dabei Fehler [10]. Weiterhin wurde bereits in den Studien von Saedon (2012) et al. und Norcini et al. (2007) vorgeschlagen, dass die Bereitstellung von Rückmeldungen aus formativen Bewertungen einen positiven Einfluss auf das Lernen und die Leistung der Ärzte hatte [11, 12]. Feedback ist somit auch für die medizinische Aus- und Weiterbildung ein wesentlicher Bestandteil. Es hilft dem angehenden Arzt, sein Potenzial in verschiedenen Phasen des Trainings zu maximieren, sein Bewusstsein für Stärken und Schwächen zu schärfen und Maßnahmen zu identifizieren, die zur Verbesserung der Leistung ergriffen werden müssen [11, 13, 14]. Ohne den kritischen Schritt des Feedbacks kann kein Aufstieg zum Niveau eines kompetenten Arztes erfolgen [10, 15].

Obwohl die Notwendigkeit von Feedback bei der medizinischen Ausbildung bekannt ist, deuten Studien darauf hin, dass Rückmeldungen zu selten erfolgen und / oder von geringer Qualität sind [16-24]. Umfragen unter Studierenden zeigen ebenso, dass Feedback bei Diskussionen zwischen Lehrern und Lernenden allzu oft fehlte oder unzureichend war [22]. Richtig durchgeführt, verbesserte Feedback aber einerseits die Beziehung zwischen Lehrern und Lernenden und führte andererseits zu vorteilhaften Änderungen im Verhalten der Lernenden [22].

\section{Peer-assisted Learning}

In einer früheren Arbeit von Ochsendorf et al. zeigte sich, dass im Dermatologie-Praktikum auch in Kleingruppen nur 50\% der Studierenden ein explizites Feedback von ihren Tutoren erhielten [4]. Zudem hatten mehrere Studien nachgewiesen, dass Peer-assisted Learning [6, 7], d.h. Unterricht durch Studierende mit ähnlichem Ausbildungsniveau, zu positiven Lernerfolgen sowohl beim Lehrenden als auch Lernenden führen. Daher wurde ein Konzept entwickelt, bei dem sich die Studierenden gegenseitig Feedback geben sollten. 
Das gegenseitige Feedback sollte die Fähigkeit fördern, einen fremden Befund strukturiert zu analysieren, sowie den Kommilitonen Feedback zu geben. Statt bisher 2 Befundbeschreibungen zu erstellen, konnten Studierende 2 weitere Befundbeschreibungen schreiben, lesen und analysieren. Die gegenseitige Bewertung erlaubte es, den strukturierten Ablauf öfter zu trainieren. Zudem bestand die Hoffnung, dass eine Rückmeldung von einem Studierenden leichter akzeptiert werden könnte als vom Tutor. Nach Han et al. soll das Peer-assisted Learning als eine Form des kooperativen Lernens ein aktiveres Engagement der Studierenden im Lernprozess fördern [25]. Studierende wurden durch diesen Ansatz zudem dazu gebracht, selbst Feedback zu geben, was künftig in ihrem Beruf hilfreich sein könnte.

Es wurden bereits Studien und Analysen durchgeführt bezüglich des Erfolges von Feedback zwischen Studierenden derselben Gruppe, welche insgesamt eine Verbesserung der Entwicklung, der akademischen Fähigkeiten sowie einen positiven Einfluss auf die Studierenden gezeigt hatten. Beispielsweise kamen Van Zundert, Sluijsmans und Van Merriënboer [26] in einer Übersichtsarbeit zu dem Schluss, dass gegenseitiges Feedback zwischen Studierenden die Entwicklung domänenspezifischer Fähigkeiten anregen konnte. Weiterhin zeigte sich auch in einer Meta-Analyse von Bart Huisman et al., dass Studierende ihr akademisches Schreiben stärker verbesserten, wenn gegenseitiges Feedback zwischen Studierenden stattfand [27].

\subsubsection{TECHNISCHE LÖSUNG}

In den Studien von Ballantyne [28] und Tahir [29] wurde jedoch beobachtet, dass es Schwierigkeiten bei persönlichem Feedback unter Studierenden gab. Diese Studie ergab, dass bei direktem Feedback die Gefahr besteht, dass die Studierenden eher positive Beurteilungen schreiben, um negative Folgen für das Sozialleben zu vermeiden. Das Feedback sollte daher anonym erfolgen, um eine offene und transparente Beurteilung durch die Studierenden zu erreichen.

Bei Durchführung eines anonymen Feedbacks in Papierform, bestünde allerdings die Möglichkeit, dass Studierende die Handschrift eines Kommilitonen erkennen könnten, wodurch wieder die beschriebene 
Verzerrung auftreten könnte. Zudem könnte es bei schwer leserlichen Handschriften auch zu Verständnisproblemen kommen, so dass der Lerneffekt wegfallen würde. Dies verstärkte die Entscheidung für die Entwicklung einer Webanwendung.

Die Studierenden sind mit dem Internet vertraut und den Umgang mit digitalen Medien gewohnt.

Die Möglichkeiten, welche die virtuelle Technologie für das Lernen bietet, ergeben sich aus den Hauptmerkmalen und Vorteilen, die in den E-LearningWebanwendungen eingesetzt werden können [30, 31]. Das Internet und die Webanwendungen bieten viele Möglichkeiten, v.a. asynchrones Lernen [32, 33].

Mehrere Studien der letzten Dekade weisen entweder auf eine Überlegenheit oder zumindest eine Gleichwertigkeit von E-Learning im Vergleich zu konventionellem Lernen hin. Dies wurde auch belegt durch mehrere MetaAnalysen. Beispielsweise zeigte eine Meta-Analyse von Cook et al. [34] aus dem Jahr 2008, dass internet-basiertes Lernen verglichen zu keiner Intervention mit großen positiven Effekten verbunden war, Allerdings waren die Auswirkungen im Vergleich zu Unterrichtsmethoden ohne Verwendung des Internets heterogen und im Allgemeinen gering, was auf eine ähnliche Wirksamkeit wie bei herkömmlichen Methoden hindeutet. Eine andere MetaAnalyse von Means et al. aus dem Jahr 2013 [35] sprach dafür, dass ELearning im Vergleich zu konventionellem Unterricht zu zusätzlicher Lernzeit, mehr Unterrichtsressourcen und Kurselementen sowie zur Interaktion zwischen den Lernenden führte. In dem Zeitraum unserer Untersuchung beschrieben Pradeep et al. 2014, dass Online-E-Learning in der Ausbildung von Medizinstudierenden dem traditionellen Lernen gleichwertig und teilweise sogar überlegen war [36]. In dieser systematischen Übersichtsarbeit wurden 59 Studien eingeschlossen mit insgesamt 6750 Studierenden aus den Bereichen Medizin, Zahnmedizin, Krankenpflege, Physiotherapie und Pharmazie. Hierbei ergaben 18 Studien signifikant höhere Leistungen in den Online-E-Learning-Interventionsgruppen verglichen mit dem traditionellen Lernen, während 30 Studien keine signifikanten Unterschiede feststellen konnten. 
In einer neueren Metanalyse von Peia und Wub aus dem Jahr 2019 konnten ebenfalls nicht alle Studien eine Überlegenheit der Online-Lernmethoden nachweisen. In keiner der Studien war jedoch eine Unterlegenheit der OnlineLehrmethoden erkennbar [37].

Insgesamt konnte man zum Zeitpunkt unserer Untersuchung im Jahr 2014 davon ausgehen, dass E-Learning mindestens gleichwertig zum konventionellen Lernen in der medizinischen Ausbildung verwendet werden konnte.

Die Umsetzung sollte über eine Webanwendung erfolgen, da die Verteilung der Epikrisen viel einfacher durchführbar war als in Papierform und eine automatisierte Verteilung auch die Arbeitszeit verringerte. Mit einer elektronischen Umsetzung konnten zudem die Anonymität der Befundbeschreibungen gewährleistet werden.

Dadurch, dass die Studierenden die Epikrisen zum Korrigieren sowie die Korrekturen direkt an ihrem Computer erhielten, waren keine ZusatzPräsenztermine nötig. Ansonsten wäre es aufgrund von Krankheitsfällen, kollidierenden Terminen oder logistischen Problemen möglicherweise nicht gelungen, das gegenseitige Feedback zu organisieren.

Es war eine große Herausforderung, eine elektronische Webanwendung selbst zu erstellen. Hierfür braucht man die Kenntnisse für SoftwareEntwicklung und die technischen Voraussetzungen für ein solches System. Eines der Hauptprobleme in der Entwicklung von Software für verschiedene Branchen ist, dass sich die Software-Entwickler nicht in der jeweiligen Branche auskennen und somit die Bedürfnisse des Auftraggebers nicht verstehen. Das führt zu Kommunikationsproblemen, erhöhtem Zeitbedarf und erhöhtem Verbrauch von Ressourcen [38, 39].

Normalerweise werden diese Probleme durch Anforderungsmanagement behoben. Der Zweck des Anforderungsmanagements besteht darin, sicherzustellen, dass der Auftragnehmer die Bedürfnisse und Erwartungen seiner Auftraggeber dokumentiert, überprüft und erfüllt [40].

In diesem Fall war es daher wichtig, die Lehre in der Medizin zu verstehen. Der Software-Entwickler war in unserem Fall der Doktorand, welcher zum Zeitpunkt der Studie ebenfalls ein Medizinstudent war und langjährige Erfahrung in Informatik, unter anderem in Software-Entwicklung mitbrachte. 
Somit waren inm sowohl die Lehrmethoden im Studium der Humanmedizin bekannt als auch die Bedürfnisse der Studierenden selbst. Dadurch konnte die Kommunikation verbessert werden, der Zeitverbrauch verringert und die Komplexität reduziert werden.

\subsection{Primäres StudienZiel: ElekTRONISChe UMSetZung DeS Gegenseitigen FEEDBACKS}

Die Chaostheorie besagt, dass geringfügige Änderungen der Anfangsbedingungen dramatische Auswirkungen auf den weiteren Verlauf der Ereignisse haben können. Dies ist einer der Hauptgedanken, den man haben muss, bevor man eine Softwareentwicklung durchführen will. Im ersten Schritt sollte man sich die Struktur eines Systems und deren Algorithmen gut überlegen.

Deshalb wurde die Webanwendung nach dem Wasserfallmodell zur Softwareentwicklung von Winston W. Royce entwickelt [41, 42]. Der Fortschritt dieses Modells verläuft linear in den Phasen: Anforderungen, Konzeption, Entwurf, Analyse und Design der Softwarearchitektur, Konstruktion, Überprüfung und Wartung [43]. Dieses Modell wurde gewählt, da ich als einziger Entwickler eine klare lineare Struktur brauchte. Es sollten keine parallelen Phasen laufen, welche für mehrere Entwickler geeignet wären. Dies könnte nämlich die Struktur beeinflussen und Verwirrungen verursachen, wenn nur ein einzelner Entwickler daran arbeitet. Es gibt mehrere Argumente, warum dieses Modell verwendet wurde.

Die Zeit, die zu Beginn des Softwareproduktionszyklus aufgewendet wird, kann die Kosten in späteren Phasen senken. Zum Beispiel kann ein Problem, das in den frühen Stadien gefunden wird (z. B. die Anforderungsspezifikation), zeitgünstiger behoben werden, als wenn derselbe Fehler erst später im Prozess gefunden wird (um den Faktor 50 bis 200) [44].

Das Modell selbst ist strukturiert und verläuft linear durch diskrete, leicht verständliche und erklärbare Phasen. In der Praxis führen Wasserfallmethoden zu einem Projektplan, bei dem 20 bis $40 \%$ der Zeit für die ersten beiden Phasen (Anforderungen und Konzeption), 30 bis $40 \%$ der Zeit für die Codierung und der Rest für Tests und Implementierung aufgewendet werden. Die meisten mittleren und großen Projekte enthalten 
detaillierte Verfahren und Kontrollen, die jeden Prozess des Projektes regeln [45]. Aufgrund der Verständlichkeit und Effektivität wird das Wasserfallmodell in vielen Texten und Kursen der Softwareentwicklung als erstes Beispiel für ein Entwicklungsmodell verwendet [46].

Es gibt jedoch auch Kritik am Wasserfallmodell: Auftraggeber wissen möglicherweise nicht genau, was ihre Anforderungen sind, bevor sie die funktionierende Software sehen. Falls die Anforderungen geändert werden, führt das zu einer Neugestaltung, Neuentwicklung und erneuten Prüfung, was wiederum auch zu erhöhten Kosten führt [47].

Ein weiteres Problem bei Verwendung des Wasserfallmodels ist, dass sich Entwickler beim Entwerfen eines neuen Softwareprodukts zukünftiger Schwierigkeiten noch nicht bewusst sind [48].

Die Kritikpunkte des Wassermodells waren für diese Arbeit aber nicht relevant, da das Konzept und die Struktur der Webanwendung deutlich definiert waren. Es war somit nicht nötig das Design der Webanwendung im Verlauf zu ändern.

\subsubsection{ANFORDERUNGEN AN DIE WEBANWENDUNG}

Die Webanwendung musste ein elektronisches Werkzeug sein, welches für alle Studierenden immer zur Verfügung steht, sowie einfach und klar zu bedienen ist. Die Webanwendung sollte auch alle von den Studierenden eingetragenen Daten zugänglich machen, so dass diese Daten ausgewertet werden konnten.

\subsubsection{VoRAUSSETZUNGEN DER WEBANWENDUNG IN BEZUG AUF DIE}

\section{STUDIERENDEN}

Das Programm sollte ohne besondere Kenntnisse in elektronischer

Datenverarbeitung nutzbar sein. Die Studierenden mussten lediglich in der Lage sein, Daten am Rechner einzutragen und zu bearbeiten. Diese Fähigkeit konnten wir voraussetzen, da die Studierenden seit Beginn des Medizinstudiums bereits wiederholt einen Rechner benutzen mussten, um verschiedene Hausaufgaben und Recherchen für Kurse zu absolvieren sowie Lernmaterial herunterzuladen. Zudem hatten die Studierenden zu diesem Zeitpunkt bereits die Kurse Biometrie und EBM abgeschlossen, welche am 
Computer erfolgten. Die Webanwendung sollte rund um die Uhr mit jedem PC aufrufbar sein.

Die Webanwendung sollte somit folgende Voraussetzungen für die Studierenden erfüllen:

- Benutzerfreundlichkeit

- Zugänglichkeit

- Erreichbarkeit

\subsubsection{VORAUSSETZUNGEN DER WEBANWENDUNG IN BEZUG AUF DEN} TECHNISCHEN BEDARF

Für das Konzept des gegenseitigen Feedbacks wurde ein System benötigt, das den interaktiven Austausch und die Bewertung von Daten (Epikrisen) zwischen Studierenden ermöglicht. Dieser Austausch von Daten sollte zudem anonym erfolgen. Hierfür war eine Datenbank nötig, auf welcher die Studierenden ihre Daten speichern und abrufen konnten. Für diesen Zweck eignet sich eine sogenannte Client-Server-Architektur, also ein Kommunikationsnetzwerk mit einem Kernserver, aus dem der Benutzer Daten von einem Client abrufen und versenden kann [49].

Hierfür benötigt man zunächst ein Netzwerk, in unserem Fall das Internet. Andere Optionen wären ein geschlossenes oder ein privates Netzwerk gewesen. Dafür wären jedoch ein größerer Aufwand und weitere Programme und Installationen nötig gewesen, so dass darauf verzichtet wurde. Die Clients entsprechen einem Rechner des Benutzers, welcher mit einer einfachen Bedienung der Website diese Operationen durchführt [49]. Für diesen Zweck musste die Belastbarkeit in Bezug auf Datenübertragung, sowie die Sicherheit des Netzwerkes und die Speicherung der Daten im Vordergrund stehen.

\section{Belastbarkeit in Bezug auf Datenübertragung:}

Die Webanwendung sollte belastbar sein in Hinsicht auf die maximale Datenübertragung pro Zeiteinheit. Die maximale Datenübertragung wird erreicht, wenn alle Studierenden und auch Administratoren gleichzeitig mit der Webanwendung interagieren. 


\section{Netzwerksicherheit:}

Die Webanwendung sollte zu jedem Zeitpunkt aufrufbar sein. Es durften weder technische Fehler noch Programmierfehler auftreten, welche die Operabilität und das Zugreifen auf die Webanwendung beeinflusst hätten. Dementsprechend müssen die Algorithmen der Webanwendung sowie die technischen Umsetzungen nicht nur einwandfrei laufen, sondern mit einer adäquaten Netzwerksicherheit und Notfallplanung bei Serverausfällen ausgestattet sein.

\section{Speicherung und Datenbank:}

Die eingetragenen Daten der Studierenden sollten selbstverständlich im Server der Webanwendung gespeichert werden. Die beste Datensicherung hierfür war eine strukturierte Datenbank. Diese Datenbank sollte sowohl die von den Studierenden eingetragenen Daten für sie selbst als auch für den Administrator wieder abrufen können. Die Struktur und die Verwendbarkeit sollten auf beiden Seiten (Studierenden und Administrator) dynamisch und so einfach wie möglich sein, um Komplexitäten auf einem Minimum zu reduzieren [50, 51].

Die Datenbank sollte auch die Möglichkeit haben, die eingetragenen Daten in die bekanntesten Tabellenkalkulationsprogramme (beispielsweise Microsoft Excel $\AA$, OpenOffice.com Calc $\AA$ und Number von Apple $\AA)$ zu exportieren, um diese dort weiter zu bearbeiten.

\subsubsection{HERAUSFORDERUNGEN}

Hinsichtlich der technischen Umsetzung bestanden in diesem Projekt folgende große Herausforderungen für die Entwicklung eines elektronischen Werkzeugs:

- Erstellung einer vernünftigen Struktur für die Datenbank.

- Entwicklung eines Algorithmus für das gegenseitige Feedback.

- Freischaltung von Inhalten je nach Zeitpunkt in den entsprechenden Fristen.

- Synchronisation der Daten.

- Gewährleistung einer einwandfreien Funktionalität in allen Zeiträumen.

- Optimierte Datensicherung, auch in Notfallsituationen.

- Exportierbarkeit der Daten zur weiteren Verwendung. 


\subsubsection{ENTSCHEIDUNG FÜR EINE WEBANWENDUNG}

Wir entschieden uns für eine Webanwendung, da jeder Benutzer (in diesem Fall Studierender) nur einen Rechner mit Internet Zugriff auf die World Wide Web und einem Webbrowser brauchte. Alle Studierenden verfügten entweder selbst über einen Rechner oder hatten Zugriff auf einen Rechner in der Universitätsbibliothek der Universität Frankfurt am Main. Zudem gehört der Webbrowser gemäß dem Avast $\circledast$ PC Trends Report 2017-2019 zu den am meisten installierten Programmen an einem Computer [52, 53].

Eine Webanwendung kann unabhängig vom Betriebssystem betrieben werden und es sind keine weiteren Installationen notwendig.

Damit wurden die Barrieren zwischen Betriebssystemen und verschiedenen Rechnertypen überwunden, da alle diese Rechner und Betriebssysteme über einen Webbrowser verfügen, der immer dieselbe Art von Dateien (HTML) aus den Webseiten liest, und in gleicher oder ähnlicher Weise (je nach Webbrowser) interpretiert.

Der Unterschied zwischen der Interpretation dieser Webseiten in den verschiedenen Webbrowsern, ist lediglich die optische Darstellung. Der Inhalt bleibt gleich. Es wurde bei der eigenen Webanwendung darauf geachtet, dass alle gängigen Browser die Seite korrekt darstellen.

\subsubsection{Hardware der Webanwendung: Server} Entscheidung für einen externen Webserver:

Für die Wahl des Webservers, im technischen Sinn Hardware, wurde eine private Option gewählt. Einen Webserver vom Hochschulrechenzentrum zu mieten oder zur Verfügung gestellt zu bekommen, hätte viele vor allem bürokratische Einschränkungen aufgrund der Sicherheit des Universitätsnetzwerks gehabt. Dieses muss nämlich in mehreren Schnittstellen für die vielen Rechner und Anwendungen der Universität geschützt werden. Eine vollkommen freie Verwaltung des Webservers könnte aus demselben Grund nicht gewährleistet werden. Andererseits wäre dann auch kein freier, vollständiger und sofortiger Zugriff möglich gewesen, um technische Notfälle lösen zu können. Der Doktorand hatte zudem bereits einen Webserver gemietet, der diese Voraussetzungen erfüllte. 


\section{Gewährleistung einer einwandfreien Datenübertragung:}

Der Server sollte in der Lage sein, die Dateninformation schnell genug zu bedienen, ohne abzustürzen. Da es sich bei dem Datenaustausch um reine Texte ohne Medien (Bilder und Videos) handelte, gab es keinen Bedarf für eine hohe Geschwindigkeit der Datenübertragung.

In dem Zeitraum der Durchführung dieser Untersuchung war die mittlere Geschwindigkeit der Datenübertragung im Internet in Deutschland ca. 8 Megabytes pro Sekunde und die niedrigste Datenübertragung war 1 Megabyte pro Sekunde bei mindestens 96\% der Verbindungen bundesweit $[54,55]$. Zusätzlich waren die Daten, die von den Studierenden ausgetauscht wurden, nicht größer als 0,05 Megabyte und die Daten innerhalb der Datenbank maximal 0,2 Megabytes. Die Datenübertragungsrate unseres ausgewählten Servers lag bei ca. 16 Megabytes pro Sekunde. Somit waren Größe und Geschwindigkeit des Datenaustausches zwischen Studierenden sowie die im Hintergrund laufenden Prozesse gewährleistet.

\section{Spiegelserver als Sicherheitsserver:}

Es wurde ein zweiter Server von einer anderen Firma in einem anderen Bundesland in Deutschland zur Verfügung gestellt. Dieser Server sollte als Spiegelserver oder Sicherheitsserver dienen für den Fall, dass der Hauptserver ausfällt und die Informationen nicht mehr abrufbar wären oder verloren gehen würden.

Spiegelserver sind Repliken einer anderen Website oder eines beliebigen Netzwerkknotens. Das Konzept der Spiegelung gilt für Netzwerkdienste, auf die über ein beliebiges Protokoll wie HTTP oder FTP zugegriffen werden kann. Solche Websites haben andere URLs als die ursprüngliche Website, enthalten jedoch identische oder nahezu identische Inhalte [56].

Spiegelstandorte befinden sich häufig in einer anderen geografischen Region als der ursprüngliche oder vorgelagerte Standort. Der Zweck von Spiegeln besteht darin, den Netzwerkverkehr zu reduzieren, die Zugriffsgeschwindigkeit zu verbessern, die Verfügbarkeit des ursprünglichen Standorts aus technischen Gründen sicherzustellen oder eine Echtzeitsicherung des ursprünglichen Standorts bereitzustellen [57]. Um die Funktionalität des Spiegelservers im Rahmen der Datensicherung zu gewährleisten, wurden die Daten im Sekunden-Takt vom Hauptserver zum 
Sicherheitsserver kopiert. Dieser Sicherheitsserver, welcher eine ähnlich hohe Datenübertragungsrate hatte wie der Hauptserver, überprüfte die Funktionalität vom Hauptserver mittels einer in PHP selbstprogrammierten Anwendung. Falls es zu einem Ausfall des Hauptservers kommen würde, dann würde die Internet-Adresse automatisch auf den Sicherheitsserver umgeleitet werden.

Zusätzlich hat der Doktorand mit einer selbstprogrammierten Software eine Datensicherung im Stundentakt auf seinen lokalen Rechner durchgeführt. Am Ende des Tages erfolgte zusätzlich eine Sicherung all dieser Backups auf einem externen Speichermedium.

Die Entscheidung für einen Server in einem anderen Bundesland wurde getroffen, um das Aufrufen des Servers auch dann zu garantieren, falls es regionale Probleme gäbe.

\subsubsection{Software: Serversoftware, Programmiersprache und Datenbank}

Für eine Webanwendung im Internet wird nicht nur ein Server (Hardware), sondern auch die Software benötigt, um diese zu bedienen, ein sogenannter Webserver. Ein Webserver ist ein Server, der Dokumente an Clients, in diesem Fall Webbrowser, überträgt.

\section{Serversoftware und Programmiersprache:}

Die meistbenutzte Webserver-Software im Internet ist Apache HTTP Server $®$ [58]. Dieser ist ein frei zugängliches, kostenloses Produkt [59] und hat einen sehr guten Ruf in Bezug auf die Sicherheit [60].

Die Grundlage des World Wide Web sind HTML-Dokumente (Hypertext Markup Language). Die Erstellung und die Kenntnisse für die Codierung dieser Dokumente sind eine Grundvoraussetzung für das Aufbauen einer Webanwendung im World Wide Web [61].

Diese Webanwendung ist in engerem Sinn eine Webseite, welche für die eigene Untersuchung dynamisch sein musste. Die HTML-Dokumente haben keine Dynamik, da es sich um statische Dokumente handelt. Man braucht somit zusätzliche Komponenten, um die Funktionalität zu erweitern, nämlich Skriptsprachen und für komplexere Funktionen auch Programmiersprachen. Um Kosten zu reduzieren, wurde eine kostenlose, frei zugängliche Programmiersprache und Datenbank gewählt. Die PHP-Programmiersprache 
erfüllt diese Voraussetzungen und ist bekannt für eine große

Datenbankunterstützung [62] sowie zahlreiche Funktionsbibliotheken [63].

Zudem war der Doktorand seit Jahren mit dieser Programmiersprache vertraut.

\section{Datenbank:}

Es wurde nicht nur eine Datenbank benötigt, welche die Daten speichert, sondern eine, welche mehrere Tabellen mit verschiedenen Elementen (in unserem Fall Daten der Studierenden, Epikrisen, Korrekturen und Fristen) speichern kann. Da diese Daten in Zusammenhang stehen, ist es eine komplexe Aufgabe für die Datenbank, alle diese Daten zu verbinden. Konventionelle Datenbanken sind dafür nicht ausreichend, so dass eine relationale Datenbank verwendet wurde, welche bei zusammenhängenden Daten besser geeignet ist.

Eine relationale Datenbank ist eine digitale Datenbank, die auf dem im Jahr 1969 von E.F. Codd präsentierten, relationalen Datenmodell basiert [64]. Das relationale Datenmodell ist ein Ansatz zur Verwaltung von Daten unter Verwendung einer Struktur und Sprache, die mit der Prädikatenlogik erster Ordnung (zum Beispiel Identifikationsnummer) übereinstimmt [64, 65]. Alle Daten werden dabei in Tupeln dargestellt, welche in Beziehungen gruppiert sind.

Dieses relationale Datenbankmodell benötigt eine Software zu ihrer Verwaltung. Viele relationale Datenbankverwaltungssysteme haben die Möglichkeit, SQL (Structured Query Language) zum Abfragen und Verwalten der Datenbank zu verwenden [66]. Deshalb wurde die kostenlose, frei zugängliche Software MySQL gewählt, welche zudem eine der am meisten verbreiteten weltweit ist [67]. MySQL wird am häufigsten für Webanwendungen verwendet und kann in Verbindung mit einem ApacheHTTP-Server und in Verbindung mit PHP-Programmiersprache eingesetzt werden. Für die Bedienung dieser Datenbank war die Beherrschung eines sogenannten SQL-Skriptes nötig. Ein weiterer wichtiger Grund für diese Entscheidung war die kostenfreie Webanwendung phpMyAdmin, welche zur Administration von MySQL-Datenbanken dient und einfach zu installieren und zu bedienen ist. Für die Verwendung von phpMyAdmin sind zwar Kenntnisse von SQL-Skript nötig, aber die Webanwendung besitzt eine 
benutzerfreundliche graphische Oberfläche, welche die Bedienung erleichtert und beschleunigt.

\subsubsection{TESTPHASE DER WEBANWENDUNG}

Die Webanwendung sollte auf 2 Ebenen getestet werden, einerseits auf technischer Ebene im Hintergrund (Programmierung und Serverfunktionalität) andererseits auf der Benutzeroberfläche (Benutzbarkeit und Funktionalität der Webanwendung für die Studierenden). Hierzu wurde der Box-Ansatz, welcher in White- und Black-Box-Tests unterteilt wird, eingesetzt.

Die Testung der Funktionalität der Webanwendung in Bezug auf die Hintergrundprozesse erfolgte anhand der White-Box-Methodik. Damit sollte die Funktionalität des Servers, des Sicherheitsservers und der Programmierung überprüft werden.

Beim White-Box-Test werden eine interne Perspektive des Systems sowie Programmierkenntnisse verwendet, um Testfälle zu entwerfen [68-70]. Der Tester wählt Eingaben aus, um Pfade durch den Code zu üben und die erwarteten Ausgaben zu bestimmen. White-Box-Tests können auf Geräte-, Integrations- und Systemebene des Software-Testprozesses angewendet werden. Diese White-Box-Tests haben mehrere Vorteile, unter anderem sind sie einfach zu automatisieren und helfen hinsichtlich einer Optimierung des Programmier-Codes [71]. Die Tests sind leider sehr komplex, da der Tester Kenntnisse über das Programm haben muss [71, 72]. Da hier jedoch der Doktorand alle Programmier-Codierungen selbst entwickelt hatte und gründlich kannte, bestand kein Problem hinsichtlich einer Durchführung eines White-Box-Tests.

Alle Funktionen der Webanwendung wurden zunächst einzeln getestet, dann miteinander verbunden. Nachdem die Webanwendung fertig entwickelt worden war, wurden die Testphasen durchgeführt, die unter dem Punkt 2.5.8 Testphase erwähnt wurden.

Das Testen der Webanwendung als Benutzer erfolgte manuell, um näher und realistischer simulieren zu können, welche Probleme und Einschränkungen die Studierenden mit der Benutzung der Webanwendung haben könnten. Dafür wurde ein Black-Box-Test verwendet, für welchen im Gegensatz zum 
White-Box-Test keine Kenntnisse des Programmes nötig sind. Hierbei wurde die Verwendung der Webanwendung durch mehrere Benutzer überprüft. Black-Box-Tests sind Methoden zum Testen von Software, mit denen die Funktionalität einer Anwendung untersucht wird, ohne einen Blick auf ihre internen Strukturen oder Funktionen zu werfen. Diese Testmethode kann praktisch auf alle Ebenen des Softwaretests angewendet werden: Einheit, Integration, System und Akzeptanz [70, 71, 73].

Spezifische Kenntnisse des Anwendungscodes, der internen Struktur und der Programmierkenntnisse im Allgemeinen waren, wie bereits erwähnt, dafür nicht erforderlich [68, 71]. Der Tester weiß, was die Software tun soll, weiß aber nicht, wie sie es tut. Dem Tester ist beispielsweise bekannt, dass eine bestimmte Eingabe eine bestimmte, unveränderliche Ausgabe zurückgibt, er weiß jedoch nicht, wie die Software die Ausgabe überhaupt erzeugt [71, 74]. Der Vorteil dieser Methode ist, dass diese zu unvoreingenommenen Resultaten führt, da Entwickler und Tester unabhängig voneinander sind. Zudem zeigt diese Testmethode Probleme auf, die auf Seite des Benutzers auftreten können und dem Entwickler gar nicht bewusst wären, weil er bestimmtes Wissen eventuell vorausgesetzt hatte.

Diese manuellen Tests führten die bereits erwähnten sieben Informatiker (siehe 2.6.5 Testphase) als Test-Benutzer für die Webanwendung durch. Es war wie bereits beschrieben wichtig, dass die Test-Benutzer nur über die Funktionalität der Anwendung wussten und nicht von den Datenbanken und dem Programmiercode. Dadurch konnten sie die Webanwendung unabhängig testen und konnten feststellen, ob Anwendungsprobleme auftraten. Nach erfolgreichem Abschluss der Testphasen waren keine Änderungen der Webanwendung nötig, weder im Rahmen des technischen Hintergrundes noch im Rahmen der Benutzeroberfläche. Es konnte daher davon ausgegangen werden, dass die zuvor gut überlegte Projektplanung geholfen hatte, spätere Änderungen an der Webanwendung zu vermeiden.

\subsubsection{EINSATZ ONLINE}

Aufgrund des gut überlegten Ansatzes, liefen alle technischen Prozesse wie geplant. Zusätzlich konnten aufgrund der verwendeten, kostenlosen Software die Kosten gering gehalten werden. Diese beschränkten sich lediglich auf die 
Miete für den Server. Die Kosten für die Entwicklung der Webanwendung entfielen im Rahmen dieses akademischen Projektes.

\subsection{SeKUNDÄRE StUdiENZIELE}

Neben der elektronischen Umsetzung des gegenseitigen Feedbacks stellte sich die Frage, anhand welcher Parameter der Nutzen dieses Konzepts erfasst werden konnte.

Zunächst bestand die Möglichkeit, die Resultate der Abschlussklausur mit MC Fragen auszuwerten hinsichtlich der Unterschiede zwischen Kontroll- und Experimentalgruppe. Damit sollte überprüft werden, ob das Verwenden dieser Webanwendung eine Wirkung auf den Lerneffekt der Studierenden hatte.

Zusätzlich sollte aber auch erfasst werden, ob der Einsatz von gegenseitigem Feedback zu einer konkreten Verbesserung der Fähigkeit zur

Befundbeschreibung führte. Frühere Änderungen im Dermatologie-Praktikum hatten dies bisher nicht erreichen können. Um beurteilen zu können, welchen Einfluss das gegenseitige Feedback auf die Befundbeschreibung hat, wurde die Bearbeitung eines Patientenbeispiels (Abschlussfall) durch Kontroll- und Experimentalgruppe ausgewertet. Diese Abschlussfall-Epikrise hatte dieselbe Struktur wie die Epikrise der Webanwendung, so dass die selbst erstellte Befundbeschreibung und Struktur im Ablauf erfasst werden konnte. Es ist klar, dass ein einzelner Fall hier keine weiterreichenden Schlüsse erlaubt, aber sollte bereits bei einem Fall eine Tendenz in die richtige Richtung zu sehen sein, könnte das Konzept weiter untersucht werden.

\subsubsection{MITARBEIT DER EXPERIMENTALGRUPPE}

Lediglich 34\% der Studierenden in der Experimentalgruppe hatten eine Epikrise korrigiert und kein Studierender hatte beide Korrekturen angefertigt. Somit hatte kein Studierender korrekt am gegenseitigen Feedback teilgenommen, so wie es beabsichtigt gewesen war! Durch Kontakte zwischen den Studierenden war bekannt geworden, dass einige Studierende (Experimentalgruppe) zusätzliche Aufgaben erhalten hatten. Die Studierenden der Experimentalgruppe beklagten sich daher beim Doktoranden, dass sie es unfair fanden, zusätzliche Aufgaben mit der Webanwendung erledigen zu müssen, ohne dass das eine Konsequenzen für 
die Note hätte. Es ist anzunehmen, dass dies einen großen Einfluss auf die Teilnahmebereitschaft der Studierenden am gegenseitigen Feedback hatte.

Leider war die Teilnahme am Feedback-Modell damit nicht so wie geplant. Daher konnten die sekundären Ziele eigentlich nicht interpretiert werden. Der Vollständigkeit halber werden diese aber dennoch hier kurz diskutiert.

\subsubsection{ABSCHLUSSKLAUSUR (TED- KLAUSUR)}

Bei Wiederholungsversuchen wurde nur die Punktzahl des ersten Versuches für die Studie berücksichtigt. Da die erste Klausur zeitlich näher am Kurs und somit an der Online-Epikrise lag, musste man davon ausgehen, dass Studierende, die die Klausur nicht bestanden haben, anschließend ein intensiveres Eigenstudium durchgeführt haben, um die Klausur beim nächsten Anlauf zu bestehen. Daher war dieses Resultat dann eher auf das Eigenstudium zurückzuführen als auf das gegenseitige Feedback-Modell. Hinsichtlich des Bestehens der TED-Klausur gab es keinen signifikanten Unterschied zwischen Kontrollgruppe und Experimentalgruppe, allerdings zeigte sich ein signifikanter Unterschied bei der erreichten Punktzahl. Die Experimentalgruppe hat eine um 5,44\% höhere Punktzahl erreicht als die Kontrollgruppe.

Insgesamt haben jedoch nur 60\% der Studierenden in der Experimentalgruppe die Epikrise Online eingetragen und nur ein Drittel eine weitere Epikrise beurteilt. Ob diese Studierenden dadurch einen Lernvorteil hatten, ist unklar. Zumindest mussten sich die Studierenden jedoch durch das Eintragen der Epikrise auf der Webanwendung erneut mit dieser auseinandersetzen. Alleine das Übertragen könnte schon zu einer leichten Verbesserung der Punktzahl geführt haben. Weitergehende Interpretationen verbieten sich, da kein Studierender wirklich korrekt teilgenommen und beide Korrekturen gemacht, so wie es eigentlich vorgesehen war.

\subsubsection{EPIKRISE-ABSCHLUSSFALL}

In dem Epikrise-Abschlussfall zeigte sich ein signifikanter Unterschied in der Punktzahl zwischen Kontroll- und Experimentalgruppe. Die Studierenden der Experimentalgruppe erreichten insgesamt eine, um 4,72\% (0,66 Punkte von maximal 14 Punkten) höhere Punktzahl als die Studierenden der 
Kontrollgruppe. Auch hier kann man spekulieren, dass diese Verbesserung auch schon durch das erneute Auseinandersetzen mit der Epikrise beim Übertragen auf die Webanwendung erfolgte.

Allerdings kann wegen der Anonymität nicht nachvollzogen werden, welche Studierenden der Experimentalgruppe eine Korrektur oder keine Korrektur geschrieben hatten. Somit konnte im Nachhinein nicht mehr rückverfolgt werden, ob die Studierenden, welche eine Korrektur angefertigt hatten, besser abschnitten als diejenigen ohne Korrektur.

\subsubsection{FRAGEBOGEN ZUR SELBSTEINSCHÄTZUNG UND BEWERTUNG DES GEGENSEITIGEN FEEDBACKS}

Um mehr über die Akzeptanz der Webanwendung unter den Studierenden zu erfahren und darüber wie die Studierenden den Nutzen dieser Webanwendung einschätzten, wurde der bereits vorher verwendete Evaluationsbogen bei Abschluss des Kurses um entsprechende Fragen ergänzt.

In verschiedenen Studien bezüglich Lehrevaluation durch Studierende, wurde kein signifikanter Unterschied in der Aussagequalität zwischen anonym und personalisiert erhobenen Daten nachgewiesen [75]. Allerdings war in der zitierten Studie die Voraussetzung für eine gute Lehrevaluation durch die Studierenden, dass die Lehrevaluation in kleinen Gruppen mit direktem Feedback durchgeführt wurde. Dies war in der eigenen Untersuchung aufgrund der großen Anzahl von Studierenden und der verschiedenen Gruppen nicht praktikabel.

Zusätzlich zeigten andere Studien, wie zum Beispiel die Arbeit von Donovan et al. [76], dass Studierende offenere und nützlichere Kommentare und Evaluationen über einen Kurs schrieben, wenn diese Bewertungen anonym durchgeführt wurden [76, 77].

Aus diesem Grund erfolgte die Evaluation anonym. Die Fragebögen wurden von den Studierenden ordentlich ausgefüllt, teilweise auch mit Kommentaren versehen, was sicherlich eine Folge der Anonymität war. Man kann daher davon ausgehen, dass die Studierenden ihre ehrliche Meinung geschrieben hatten und die Daten verwertbar waren. 


\subsubsection{Selbsteinschätzung durch die Studierenden}

Bezüglich der Selbsteinschätzung vor dem Kurs zeigte sich ein signifikanter Unterschied zwischen den beiden Gruppen. Die Studierenden der Experimentalgruppe schätzten sich mit durchschnittlich 2,90 Punkten besser ein als die Studierenden der Kontrollgruppe (2,11 Punkte). Dieser Unterschied kann natürlich einerseits rein subjektiv sein, er kann jedoch auch die Realität widerspiegeln, da bei der Randomisierung kein Matching hinsichtlich der Studienleistungen erfolgte. Somit können die besseren Resultate der Studierenden der Experimentalgruppe in der Abschlussklausur und in dem Epikrise-Abschlussfall auch mit den unterschiedlichen vorbestehenden Studienleistungen zusammenhängen, unabhängig vom gegenseitigen Feedback.

In der Selbsteinschätzung bzgl. der Verbesserung der Befundbeschreibung (KG Fragenummer 7, EG Fragenummer 15) zeigten sich gute Werte (Kontrollgruppe 4,83 Punkte und Experimentalgruppe 4,49 Punkte) in beiden Gruppen, jedoch ohne signifikanten Unterschied. Dies könnte daran liegen, dass die subjektive Verbesserung der Befundbeschreibung lediglich durch den Kurs erfolgte.

Bei der Frage nach der Wirksamkeit verschiedener Elemente zeigte der Unterpunkt «Hausaufgabe» einen signifikanten Unterschied mit einer Punktzahl von 4,46 Punkten in der Experimentalgruppe und 3,92 Punkten in der Kontrollgruppe (Kontrollgruppe Fragenummer 8 im Anhang 8, Experimentalgruppe Fragenummer 17 im Anhang 9). Möglicherweise kann dies dadurch erklärt werden, dass das gegenseitige Feedback für die Studierenden auch als eine Art Hausaufgabe gewirkt hat, da sie als zusätzliche Aufgabe die Epikrise eintragen mussten sowie fremde Epikrisen korrigieren sollten.

\subsubsection{Bewertung des gegenseitigen Feedbacks durch die Studierenden}

Der Ansatz des gegenseitigen Feedbacks wurde mit einer Schulnote (deutsches Schulsystem) von 2,93 bewertet, was einer Bewertung von «befriedigend» entspricht. Möglicherweise wäre auch hier die Bewertung deutlich besser gewesen, wenn die Studierenden nicht, wie bereits erwähnt, 
das Gefühl einer unfairen Behandlung durch eine Zusatzaufgabe gehabt hätten.

Die Verständlichkeit des Feedback-Konzeptes und die Verständlichkeit der Benutzung der Lernplattform wurden überdurchschnittlich gut bewertet (siehe 3.8.2 Bewertung des gegenseitigen Feedbacks durch die Studierenden). Man kann somit davon ausgehen, dass die geringe Teilnahme nicht auf Verständlichkeitsprobleme bei der Anwendung des gegenseitigen Feedbacks oder der Lernplattform zurückzuführen sind.

Die Erklärung, warum einige Studierenden nur 1-2 Punkte vergeben hatten und sich durchschnittlich so eine tiefe Punktzahl bei der Befundbeurteilung durch das gegenseitige Feedback ergab, findet sich teils in den freien Kommentaren (siehe 3.8.2 Bewertung des gegenseitigen Feedbacks durch die Studierenden), hier waren mangelndes Feedback und die kurze Zeit der Fristen die Hauptkritikpunkte.

Die Auswertungen hinsichtlich der Selbstbewertung von Motivation für die Teilnahme am Feedback-Modell (Punkt 8 Anhang 9), Motivation durch das gegenseitige Feedback mehr zu lernen (Punkt 9 Anhang 9), Verstärkung eines Wissenszuwachs (Punkt 10 Anhang 9) und wachsende Sicherheit bei der Befundbeschreibung (Punkt 16 Anhang 9) ergab gemäß der Selbsteinschätzung der Studierenden durchschnittliche bis unterdurchschnittliche Bewertungen. Wie bereits zuvor erklärt spielte hier wahrscheinlich v.a. das Gefühl einer unfairen Behandlung zwischen Kontrollund Experimentalgruppe, eine große Rolle.

\subsection{SCHLUSSFOLGERUNGEN}

Wie bereits in der Studie «Aktivierung im klinischen Unterricht» von Ochsendorf et al. erwähnt, zeigte sich eine signifikante Verbesserung der Vorbereitungszeit der Studierenden durch freiwillige Hausaufgaben [4]. Deshalb wurde vermutet, dass die Studierenden eine ähnliche Motivation für das gegenseitige Feedback haben würden. Leider zeigte sich das nicht in den Ergebnissen.

Eine der ersten Überlegungen diesbezüglich war, dass die Motivation und Teilnahmebereitschaft aufgrund einer fehlenden Belohnung bzw. Anreizes, z.B. in Form einer Benotung, so gering waren. 
In der Metanalyse von Deci et al. wurden die Auswirkungen extrinsischer Belohnungen auf die intrinsische Motivation untersucht [78]. Extrinsische Belohnungen oder Einflüsse zeigten sich hierbei unterlegen gegenüber der intrinsischen Motivation. Positives Feedback hingegen verbesserte die Motivation [78]. Die geringe Teilnahmebereitschaft scheint daher nicht unbedingt an dieser fehlenden Belohnung gelitten zu haben. Es ist aber zu vermuten, dass das Gefühl von «Ungerechtigkeit» und "unfairer Behandlung» durch unterschiedliche Behandlung (Zusatzaufgaben) der Kontroll- und Experimentalgruppe dazu führte, dass die Motivation zur Teilnahme gering war.

Fehlende Teilnahmebereitschaft von Studierenden wurde auch in anderen Studien mit Peer-assistiertem Lernen beobachtet. In einer Studie von Sopka et al. aus dem Jahr 2015 zeigten sich Limitationen aufgrund der begrenzten Studienpopulation «Studierende» und dadurch erschwerter Durchführbarkeit von kontrollierten, randomisierten Studien [79]. Zusätzlich war auch hier die Teilnahmebereitschaft unter den Medizinstudenten gering; von 221 in der Untersuchung eingeschlossenen Studierenden wurden nur 142 erfasst und ausgewertet [79]. Diese Studie war zum Zeitpunkt unserer Untersuchung noch nicht veröffentlicht, hatte aber mit ähnlichen Problemen zu kämpfen. Es kam ebenfalls zu einer mangelnden Teilnahmebereitschaft und es zeigten sich keine signifikanten Unterschiede hinsichtlich der Benotung.

Zur tatsächlichen Feststellung einer Verbesserung des Lernverhaltens durch das gegenseitige Feedback werden jedoch sicher weitere Studien notwendig sein. Hierbei müsste man dann insbesondere auf eine größere Studienpopulation achten, um die statistische Aussagekraft zu erhöhen. Zudem müsste auch die Kontrollgruppe Aufgaben erhalten, um das bereits erwähnte Gefühl von Ungerechtigkeit zu vermeiden.

Alternativ könnten künftig „historische Kontrollen“ verwendet werden, um die Fallzahl zu vergrößern. Eine Studie aus dem Jahr 2015 zeigte, dass Studierende durch Peer-assistiertes Lernen den Inhalt eines Anatomie-Kurses als verständnisvoller empfanden und bessere akademische Ergebnisse erzielte [25]. Interessanterweise wurde in dieser Untersuchung als Kontrollgruppe ein Kurs aus dem Jahr 2008 gewählt, während die Experimentalgruppe ein Kurs im Jahr 2014 war. Durch erneutes Durchführen 
unserer Untersuchung mit Kontroll- und Experimentalgruppen in unterschiedlichen Jahren könnte dadurch evtl. das Gefühl von ungleicher Behandlung eliminiert werden. Bei einer zeitlichen Trennung von Kontroll- und Experimentalgruppe müsste man aber darauf achten, dass der Abstand nicht zu groß ist, da es sonst zum Auftreten von anderen Verzerrungen, wie anderen Dozenten oder Änderungen von Inhalten, kommen kann. Insgesamt kann man sagen, dass die Webanwendung erfolgreich entwickelt und eingesetzt werden konnte. Sie hat einwandfrei funktioniert und es gab keine Verständnisprobleme. Dieser Ansatz kann auch für andere Fächer des Studiums der Humanmedizin angewendet werden.

Trotz aller Bemühungen wurde das Konzept von den Studierenden aber nicht akzeptiert. Die Widerstände waren bei der Planung unterschätzt worden, so dass eine definitive Aussage bezüglich des Nutzens eines solchen Ansatzes hinsichtlich des tatsächlichen Lernerfolgs derzeit leider nicht möglich ist. Bei künftigen Untersuchungen muss insbesondere stärker auf die Motivation der Studierenden geachtet werden, damit eine korrekte Teilnahme erfolgt. 


\section{ZUSAMMENFASSUNG}

\section{Hintergrund}

Die Erlernung der Fähigkeit zur Befundbeschreibung im DermatologieBlockpraktikum ist trotz aller Bemühungen noch nicht optimal. Feedback hat sich in der Lehre als essenzielle Voraussetzung für den Lernprozess gezeigt, ist aber in der täglichen Lehrpraxis zuweilen schwer umsetzbar.

\section{Fragestellung}

Lässt sich im Dermatologie-Blockpraktikum das bisher fehlende Feedback bezüglich der Befundbeschreibungen durch eine digitale Lösung in Form einer Webanwendung verbessern? Führt derartiges gegenseitiges Feedback durch die Peers zu einer Verbesserung der Ergebnisse in einem Wissenstest (Multiple-Choice-Fragen) bzw. bei der Bearbeitung eines Patientenfalls? Wie beurteilen die Studierenden diesen Ansatz?

\section{Material und Methode}

Es wurden die Anforderungen an eine Webanwendung definiert, und diese mit Hilfe einer relationalen Datenbank programmiert und bezüglich vordefinierter Gütekriterien getestet, bis das System stabil lief. Im Sommersemester 2014 wurden 12 Gruppen ( $\mathrm{n}=181$ Studierende) des Blockpraktikums Dermatologie damit prospektiv untersucht. Es erfolgte eine 1:1 Randomisierung in Kontroll- und Experimentalgruppe. Durch einen organisatorischen Fehler wurde eine der Gruppen, welche als Kontrollgruppe randomisiert wurde, als Experimentalgruppe behandelt und auch so ausgewertet („As treated Analyse“). Für die Studierenden der Kontrollgruppe $(n=76)$ erfolgte das 5-tägige Dermatologie-Blockpraktikum nach Standardablauf. Generell wurden in diesem Praktikum im Rahmen der Hospitation 2 kurze Epikrisen geschrieben. Bisher hatten die Studierenden kein Feedback bezüglich dieser Epikrisen erhalten.

Die Studierenden der Experimentalgruppe ( $n=105)$ mussten diese Epikrisen zusätzlich in die Webanwendung eintragen. Nach Ablauf einer Frist von 12 Stunden wurden die Epikrisen von der Webanwendung an zwei weitere Studierende zur Korrektur verteilt. Die korrigierte Fassung wurde den Studierenden wieder zurückgeschickt. 
Neben der Abschlussklausur bearbeiteten alle Studierenden am letzten

Praktikumstag einen virtuell präsentierten Fall und füllten einen

Evaluationsbogen aus.

\section{Ergebnisse}

Die Webanwendung funktionierte bezüglich Programmierung, Speicherung, Algorithmen und Hardware einwandfrei. Weder vom System noch von Studierenden wurden Fehler oder Probleme gemeldet.

Von den 105 eingeschlossen Studierenden der Experimentalgruppe hatten nur 60 Studierende eine Epikrise in der Webanwendung eingetragen. Zudem hatten nur 34 Studierende eine Korrektur für eine fremde Epikrise angefertigt. Keiner der Studierenden hatte wie vorgesehen zwei Korrekturen angefertigt. Die sekundären Studienziele (Ergebnisse der Abschlussklausur und des Abschlussfalls) setzten gemäß des Studienansatzes zwei Korrekturen einer fremden Epikrise voraus. Somit war leider keine aussagekräftige Interpretation dieser Daten möglich. Die Auswertung der vorliegenden Daten ergab geringe Unterschiede mit besserer Punktzahl der Studierenden der Experimentalgruppe.

Die Studierenden hatten keine Probleme mit der Webanwendung und gaben an, das Konzept verstanden zu haben. Es wurde aber durch Studierende der Experimentalgruppe beklagt, dass im Vergleich zum Standardkurs zusätzliche Aktivitäten gefordert wurden.

\section{Schlussfolgerung}

Die entwickelte Webanwendung für das gegenseitige Feedback lief stabil und funktionierte gut. Ohne Kontrolle und Überprüfung wurde sie jedoch von den Studierenden nicht so genutzt wie gewünscht. Der eigentliche Nutzen muss daher in einer künftigen Untersuchung, welche diese Probleme berücksichtigt, festgestellt werden. 


\section{SUMMARY}

\section{Background:}

Despite all efforts, learning the ability to describe findings in a dermatology internship is not yet optimal. Feedback has been shown in teaching as an essential prerequisite for the learning process but is still difficult to implement in daily teaching practice.

\section{Questions:}

Can the previously missing feedback for describing findings be improved in the dermatology internship by a digital solution such as a web application? Does such peer-assisted feedback improve the results in a knowledge test (multiple choice questions) or when processing a patient case? How do the students assess this approach?

\section{Methods:}

The requirements for a web application were defined, programmed in a relational database and tested against predefined quality criteria until the system was stable. In the summer semester 2014, 12 groups ( $n=181$ students) of the internship dermatology were prospectively examined. There was a 1:1 randomization into a control and an intervention group. Due to an organizational error, one of the groups, which was randomized as a control group, was treated as an intervention group and evaluated accordingly ("As treated analysis"). For the control group students $(n=76)$, the 5-day dermatology internship was accomplished according to the standard procedure. Normally, there were 2 short epicrisis written as part of the internship. Until now, the students had received no feedback regarding these epicrisis.

The students of the intervention group ( $n=105)$ additionally had to enter these epicrisis into the web application. After a period of 12 hours, the web application distributed the epicrisis to two other students for correction. The corrected version was sent back to the students.

In addition to the final exam, all students worked on a virtually presented case on the last day of the internship and filled out an evaluation sheet. 


\section{Results:}

The web application worked perfectly in terms of programming, data-storage, algorithms and hardware. No errors or problems were reported by the system or by students.

Only 60 of the 105 included students in the intervention group entered an epicrisis in the web application. In addition to this, only 34 students made a correction for an epicrisis of other students. None of the students made two corrections as planned. The secondary outcomes (results of the final exam and the final case) require two corrections from an epicrisis of other students in accordance with the study approach. Unfortunately, therefore, no meaningful interpretation of this data was possible. The evaluation of the available data showed small differences with better scores of the students in the intervention group.

The students had no problems with the web application and indicated that they understood the concept. However, students from the intervention group complained that additional activities were required compared to the standard course.

\section{Conclusions:}

The web application for mutual feedback was stable and worked well.

However, without control and verification, it was not used as desired by the students. Therefore, the actual benefit must be determined in a future study that takes these problems in consideration. 


\title{
7. ANHANG
}

Anhang 1: Formblatt der Epikrise während der Hospitation und für den Abschlussfall

Die Epikrise ist jeweils untergliedert in Befund, Haupt-/Verdachtsdiagnose, Differentialdiagnosen sowie diagnostische Maßnahmen.

Epikrise Abschlußfall

Grund der Vorstellung und bisheriger Verlauf: siehe Fallbeschreibung

Befund

\author{
Haupt/Verdachtsdiagnose (1) \\ Differentialdiagnose (2) \\ Differentialdiagnose (3) \\ Diagnostische Maßnahmen zur Klärung der Verdachtsdiagnosen \\ Zur Klärung von (1) \\ Zur Klärung von (2) \\ Zur Klärung von (3)
}

Aktueller Therapievorschlag: entfällt, da Ergebnisse der Diagnostik unbekannt.

Auswirkung der Hauterkrankung auf den Patienten 


\section{Anhang 2: Abschlussfall}

Der 65-j. Lothar Ralf Petersen stellt sich wegen seit 4 Wochen zunehmender Hautveränderungen vor. Er ist vor einem Vierteljahr in Rente gegangen, vorher hatte er beim Finanzamt im Büro gearbeitet. Sein wesentliches Hobby ist sein großer Garten, in dem er sich viel und gerne aufhält. In den letzten zwei Wochen hat er sich aber davon ferngehalten. Der Patient ist verheiratet; seine Ehefrau arbeitet als Sekretärin in einem Architektur-Büro. Sie juckt zwar aus Sympathie mit, hat aber bisher keine Hautveränderungen bemerkt. Die beiden Söhne (34 und 29 Jahre alt) wohnen schon lange nicht mehr zu Hause. Bei ihnen sind keine Hauterkrankungen bekannt. Sie haben ein bzw. zwei Enkelkinder. Üblicherweise kommt die gesamte Familie immer am Wochenende zum gemeinsamen Kaffee in dem schönen großen Garten zusammen, aber seit 14 Tagen finden diese Besuche nicht mehr statt, weil die Schwiegertöchter Angst vor Ansteckung der Enkel haben. An weiteren Hobbies geht der Patient gerne schwimmen, was er aber im Moment nicht mehr macht. Abends ging er bis vor 10 Tagen zum Altherrenfußball und einmal in der Woche zum Skatspielen in seine Stammkneipe, aber auch das macht er momentan nicht mehr.

Am Anfang waren es nur kleine „Stippchen“ am Arm, die ihm kaum auffielen. Er dachte, das seien Mückenstiche aus dem Garten. Dann aber haben sich die Hautveränderungen ausgebreitet - und mit bloßem Oberkörper war er auch niemals im Garten. Sonst fühlt er sich eigentlich wohl. Wenn nur diese Hautveränderungen nicht so stark jucken würden. Er kann nachts nicht mehr richtig schlafen. Wenn er sich tagsüber ablenkt ist der Juckreiz auszuhalten, im Bett und wenn er zur Ruhe kommt nervt ihn das Jucken aber sehr. Sein Hausarzt hielt die Hautveränderungen für eine Allergie gegen Gräser bzw. Reaktionen auf Grasmilben, da er sich ja seit seinem Ruhestand sehr viel in seinem Garten aufhält. Eine Behandlung mit Cetirizin hat allerdings keine wesentliche Wirkung gehabt. Lokal hat er mit Hydrocortison 0,25 \% Creme behandelt, die er ohne Rezept in der Apotheke bekommen hat. Aber auch das hatte keine Besserung bewirkt.

Der Patient war bisher hautgesund. In der direkten Familie sind keine Hauterkrankungen bekannt, allerdings ist der Vater schon im Alter von 45 Jahren bei einem Unfall umgekommen. Ein Cousin (väterlicherseits) soll eine Hauterkrankung haben, angeblich eine „Schuppenflechte“, näheres ist ihm nicht bekannt.

Der Patient raucht ca. 10 Zigaretten täglich seit gut 30 Jahren - Seit dem Ruhestand sind es noch etwas mehr geworden. Des Weiteren sind erhöhte Cholesterinwerte bekannt sowie ein etwas erhöhter Blutdruck. Deshalb nimmt der Patient seit mehr als 10 Jahren BelocZoc und Simvastatin Tabletten. In den letzten Jahren hat die Erektionsfähigkeit nachgelassen, so dass er seit einem Jahr gelegentlich auch Sildenafil 50 mg Tabletten einnimmt. Seit dem Auftreten der Hautveränderungen hat er aber keinen engen Kontakt mehr mit seiner Frau gehabt. Seine Frau hat ihm ein Multivitaminpräparat sowie Gingko-Tabletten mitgebracht. Diese nimmt er aber nicht völlig regelmäßig, weil er den Sinn nicht so ganz einsieht und die Einnahme auch öfter vergisst. Seine Frau hat ihm im Dezember auch eine Fettcreme vom Aldi mitgebracht, da sie gelesen hat, dass die Haut im Alter immer trockener wird. Diese verwendet er gelegentlich morgens nach dem Duschen. 


\section{Anhang 3: Informationsblatt für Dozenten}

1. Information der Studenten: Die Studenten erhalten ein Informationsblatt mit genauen Informationen zum Ablauf des EpikriseFeedback-Konzeptes. Zusätzlich finden sie auch online diese Informationen.

2. Registrierung: Für die Teilnahme an dem Epikrise-Feedback-Konzept müssen sich die Studenten registrieren unter www.reciprocalevaluation-model.de. Die Registrierung ist jeweils von Montag $17 \mathrm{Uhr}$ bis Dienstag 20 Uhr freigeschaltet. Bei Zeitüberschreitung sollen sich die Studenten an mich wenden (die Email finden sie unter Kontakt), damit ich sie nachträglich eintragen kann.

3. Patientenuntersuchung: Die Studenten erhalten entweder Dienstag, Mittwoch, Donnerstag oder Freitag einen Patienten zugeteilt. Sie sollen Anamnese erheben und den Patienten untersuchen.

4. Epikrise schreiben: Im Anschluss an die Patientenuntersuchung müssen alle Studenten (ausgenommen diejenigen, die erst am Freitag einen Patienten erhalten haben) eine Epikrise über diesen Patienten schreiben. Dabei müssen sie einmal den Epikrise-Bogen (wie bis jetzt im Blockpraktikum geschehen) per Hand ausfüllen und einmal diesen Epikrise-Bogen online unter www.reciprocal-evaluation-model.de ausfüllen. Der Epikrise-Bogen ist in Unterpunkte gegliedert; die Studenten sollen jeden Unterpunkt möglichst ausführlich bearbeiten. Die Bearbeitungszeit für die Online-Epikrise ist begrenzt (siehe unten). Diese Aufgaben (sowohl die Online-Epikrise als auch die zweite handschriftliche Epikrise) sind laut Herrn Prof. Dr. Ochsendorf Pflicht für die Studenten und Teil der Evaluation.

5. Korrektur: Nach Ablauf der Bearbeitungszeit für die Online-Epikrise erhält jeder Student zufällig Epikrisen von zwei anderen Studenten zum Korrigieren. Dabei müssen die Studenten ausführlich kommentieren, was sie gut bzw. schlecht finden. Zusätzlich sollen die Studenten jeden Gliederungspunkt der Epikrise mit Punkten bewerten. Die Bearbeitungszeit für die Korrekturen ist ebenfalls beschränkt (siehe unten).

6. Korrekturbestätigung: Nach Ablauf der Bearbeitungszeit für die Korrekturen erhalten die Studenten die Korrekturen ihrer eigenen Epikrise und sollen diese aufmerksam durchlesen und zur Kenntnis nehmen.

7. Zweite Epikrise: Am Freitag von 13-16 Uhr müssen alle Studenten am Ende des Blockpraktikums handschriftlich eine Epikrise schreiben bezogen auf einen Fallbeispiel. Dafür erhalten die Studenten einen Fallbeispiel, einen Epikrise-Bogen sowie eine Powerpoint-Präsentation, welche Sie von mir als Powerpoint-Datei bekommen werden. Diese Powerpoint-Präsentation soll mit einem Beamer vor der Gruppe angezeigt werden. 


\section{Anhang 4: Informationsblatt für Studierenden}

\section{Erklärung des Epikrise-Feedback-Konzept}

- Neui Bitte registrieren Sie sich für die Teilnahme am Epikrise-Feedback-System ab Montag um 17 Uhr (erster Tag Ihres Dermatologie-Blockpraktikums) online unter http://www.reciprocal-evaluation-modelde. Die Registrierung ist bis Dienstag um $20 \mathrm{Uhr}$ freigeschaltet. Später wird eine Registrierung nicht mehr möglich sein!

- Während des Praktikums schreibt jeder von Ihnen wie immer im DermatologieBlockpraktikum eine Epikrise bezogen auf einen Patienten (Anamnese, Untersuchung, Verdachtsdiagnosen, diagnostisches Procedere und Auswirkungen der Erkrankung auf den Patienten).

Neu: Sie werden diese Epikrise gegenseitig beurteilen. Durch dieses Feedback sollen Sie sicherer in den o.g. Kompetenzen werden.

- An Ihrem Hospitationstag wird Ihnen ein Patient zugeteilt. Im Anschluss an Anamnese und körperliche Untersuchung schreiben Sie (wie immer im Dermatologie-Blockpraktikum) eine Epikrise und geben diese noch am selben Tag schriftlich Ihrem Dozenten.

- Neu: Dieselbe Epikrise tragen Sie an Ihrem Hospitationstag bis spätestens 23:59 Uhr zusätzlich unter www.reciprocal-evaluation-model.de online ein (möglichst ausführlich in die jeweiligen vorgegebenen Felder).

- Neui Jeder von Ihnen erhält im Anschluss online jeweils Epikrisen von 2 anderen Studenten zum korrigieren. Bitte lesen Sie diese Epikrisen aufmerksam durch und versuchen Sie zu beschreiben, was Ihnen an diesen Epikrisen besonders gut bzw. nicht gefällt. Dazu finden Sie neben dem jeweiligen Abschnitt der Epikrise Platz für Korrekturen und Verbesserungsvorschläge.

- Neu: In die jeweiligen kleinen Kästchen oberhalb des Korrekturfeldes bitten wir Sie, eine Bewertung anhand von Punkten einzutragen. Je nach Gliederungspunkt der Epikrise können unterschiedlich viele Punkte vergeben werden; die maximale Punktzahl ist jeweils neben dem Kästchen ersichtlich. Seien Sie hierbei bitte fair und ehrlich, damit Sie anderen Studenten gegebenenfalls die Möglichkeit geben, sich verbessern zu können. (Wichtig: Die Punkte, die Sie vergeben können haben keinen Einfluss auf Ihre Note!) Damit das Modell funktionieren kann, bitten wir Sie, Ihren Mitstudierenden spätestens bis zum Folgetag um 13:00 Uhr ein Feedback zu geben (Kommentare sowie Punktevergabe).

- Am Ende des Dermatologie-Praktikums werden Sie am Freitag noch eine weitere Epikrise schreiben, anhand derer wir feststellen wollen, ob unsere Bemühungen erfolgreich waren.

Weitere Informationen (Zeiträume und Deadlines) befinden sich auf der Plattform (http://www.reciprocal-evaluation-model.de) unter dem Punkt „Information“ (Achtung: Dieser Punkt ist erst nach der Registrierung sichtbar!!).

Wir danken Ihnen für die Teilnahme am Epikrise-Feedback-System und hoffen, dass wir dadurch Ihren Lernerfolg verbessern können!

Bei Fragen melden Sie sich bitte jederzeit unter daniel chavez@me.com 


\section{Anhang 5: Zeiträume und Friste (Deadlines)}

\section{Zeiträume und Deadlines / Zusammenfassung:}

\section{Gruppe A:}

- Patient wird am Dienstag zugeteilt

- Zeitraum 1: Dienstag 8.00-23.59 Uhr => Schreiben der Online-Epikrise

- Deadline 1: Dienstag 23.59 Uhr => Bearbeitung der Online-Epikrise nicht mehr möglich

- Zeitraum 2: Dienstag 23.59 Uhr bis Mittwoch $13.00 \mathrm{Uhr}=>$ Korrektur der beiden Epikrisen

- Deadline 2: Mittwoch 13.00 Uhr => Bearbeitung der Korrekturen nicht mehr möglich

- Zeitraum 3: Freitag 13.00-16.00 Uhr => Schreiben der zweiten Epikrise bezogen auf einen Fallbericht

\section{Gruppe B:}

- Patient wird am Mittwoch zugeteilt

- Zeitraum 1: Mittwoch 8.00-23.59 Uhr => Schreiben der Online-Epikrise

- Deadline 1: Mittwoch 23.59 Uhr => Bearbeitung der Online-Epikrise nicht mehr möglich

- Zeitraum 2: Mittwoch 23.59 Uhr bis Donnerstag 13.00 Uhr => Korrektur der beiden Epikrisen

- Deadline 2: Donnerstag 13.00 Uhr => Bearbeitung der Korrekturen nicht mehr möglich

- Zeitraum 3: Freitag 13.00-16.00 Uhr => Schreiben der zweiten Epikrise bezogen auf einen Fallbericht

\section{Gruppe C:}

- Patient wird am Donnerstag zugeteilt

- Zeitraum 1: Donnerstag 8.00-23.59 Uhr => Schreiben der Online-Epikrise

- Deadline 1: Donnerstag 23.59 Uhr => Bearbeitung der Online-Epikrise nicht mehr möglich

- Zeitraum 2: Donnerstag 23.59 Uhr bis Freitag 13.00 Uhr => Korrektur der beiden Epikrisen

- Deadline 2: Freitag 13.00 Uhr => Bearbeitung der Korrekturen nicht mehr möglich

- Zeitraum 3: Freitag 13.00-16.00 Uhr => Schreiben der zweiten Epikrise bezogen auf einen Fallbericht

\section{Gruppe D:}

- Patient wird am Freitag zugeteilt

- Zeitraum 1: Freitag 8.00-23.59 Uhr => Schreiben der Online-Epikrise

- Deadline 1: Freitag 23.59 Uhr => Bearbeitung der Online-Epikrise nicht mehr möglich

- Zeitraum 2: Freitag 23.59 Uhr bis Samstag 13.00 Uhr => Korrektur der beiden Epikrisen

- Deadline 2: Samstag 13.00 Uhr => Bearbeitung der Korrekturen nicht mehr möglich

- Zeitraum 3: Freitaq 13.00-16.00 Uhr => Schreiben der zweiten Epikrise bezogen auf einen Fallbericht 


\section{Anhang 6: Anleitung zum Anfertigen der Epikrise Online}

Anfertigen der Epikrise Online

Während des Dermatologie-Blockpraktikums wird jedem von euch entweder am Dienstag, Mittwoch oder Donnerstag ein Patient zugeteilt für Anamnese und körperliche Untersuchung.

Im Anschluss an das Praktikum müsst ihr online eine Epikrise über den euch zugeteilten Patienten schreiben. Dabei ist es wichtig, auf den Grund des Klinikaufenthaltes, den bisherigen Verlauf der Erkrankung, den Befund, die Verdachtsdiagnose, die Abgrenzung gegen andere Differenzialdiagnosen, aktuelle Therapievorschläge sowie die Auswirkung der Erkrankung auf den Patienten einzugehen; alle Punkte bitte ausführlich vervollständigen! Die Bearbeitung im Reciprocal Evaluation Model, gegliedert nach den Hauptpunkten eurer Epikrise ist für den Zeitraum 1 (Zeiträume und Deadlines siehe unten) freigeschaltet. Zwischendurch könnt ihr eure Epikrise auch speichern und zu einem späteren Zeitpunkt daran weiterarbeiten. Unten auf der Seite findet ihr ein Kästchen, welches mit "Epikrise abgeben" beschriftet ist. Durch Ankreuzen dieses Kästchens und anschließendes Speichern kann die Epikrise sofort abgegeben werden, eine weitere Bearbeitung ist dann jedoch unabhängig vom Zeitpunkt nicht mehr möglich.

Nach Ablauf der Deadline 1 ist eine Änderung der Epikrise nicht mehr möglich. Zu diesem Zeitpunkt wird die Korrekturphase (Zeitraum 2) eingeleitet: Ihr erhaltet jeweils Epikrisen von 2 anderen Studenten zum korrigieren. Lest diese Epikrisen aufmerksam durch und versucht möglichst ausführlich anzugeben, warum euch etwas an diesen Epikrisen nicht gefällt oder was ihr besonders gut findet. Dazu findet ihr neben dem jeweiligen Abschnitt der Epikrise Platz für eure Korrekturen und Verbesserungsvorschläge. Oberhalb eures Korrekturfeldes sollt ihr den jeweiligen Abschnitt der Epikrise anhand von Punkten bewerten. Je nach Gliederungspunkt der Epikrise können unterschiedlich viele Punkte vergeben werden; die maximale Punktzahl ist jeweils neben dem Kästchen ersichtlich. Die Korrekturphase endet mit der Deadline 2 , anschließend könnt ihr eure Bewertungen nicht mehr ändern. Wenn ihr die Korrekturen bereits vorher abgeben möchtet, findet ihr unten auf der Seite ein Kästchen, welches mit "Korrektur abgeben" beschriftet ist. Durch Ankreuzen dieses Kästchens und anschließendes Speichern kann die Korrektur sofort abgegeben werden. Eine weitere Bearbeitung der Korrektur ist nach der Abgabe jedoch nicht mehr möglich.

Nach Ablauf der Deadline 2 erhaltet ihr auch die Bewertungen eurer eigenen Epikrise. Lest diese Bewertungen bitte aufmerksam durch und überlegt, ob Kritik oder Lob gerechtfertigt war.

Am Freitag von $13.00 \mathrm{Uhr}$ bis $15 \mathrm{Uhr}$ (Zeitraum 3) werdet ihr eine zweite Epikrise bezogen auf einen Fallbericht von Hand schreiben. Denkt dabei an die Kritikpunkte, die ihr per Reciprocal Evaluation Model erhalten habt und wendet die Verbesserungsvorschläge an.

\section{Alle gezeigten Anleitungen für die Studierenden waren auch auf der Webanwendung jederzeit nachzulesen.}




\section{Anhang 7: Fragebogen für die Epikrise Abschlussfall}

$\begin{array}{lcl}\text { Datum: } \_ & \square \text { Mittwoch } & \square \text { Donnerstag }\end{array}$

Grund der Vorstellung und bisheriger Verlauf: siehe Fallbeschreibung

Befund (Auf die Blickdiagnose bezogen)

Haupt/Verdachtsdiagnose (1)

Differentialdiagnose (2)

Differentialdiagnose (3)

Diagnostische Maßnahmen zur Klärung der Verdachtsdiagnosen

Zur Klärung von (1)

Zur Klärung von (2)

Zur Klärung von (3)

Aktueller Therapievorschlag: entfällt, da Ergebnisse der Diagnostik unbekannt.

Auswirkung der Hauterkrankung auf den Patienten? 


\title{
Anhang 8: Fragebogen zur Selbsteinschätzung (Kontrollgruppe)
}

\begin{abstract}
Fragebogen zum Dermatologie-Blockpraktikum
Liebe Studierende,

wir versuchen Ihre Kompetenzen zur Bearbeitung dermatologischer Probleme zu verbessern. Bitte teilen Sie uns Ihre Meinung mit, durch Einsatz welcher Methoden es am ehesten zu einer Verbesserung Ihrer Befundbeurteilung kommt.
\end{abstract}

Datum:

\begin{tabular}{|c|c|}
\hline 1. & Wie alt sind Sie? \\
\hline 2. & Geschlecht \\
\hline 3. & In welchem klinischen Semester befinden sie sich derzeit? \\
\hline 4. & Wie viele Famulaturen haben Sie bereits absolviert? \\
\hline 5. & $\begin{array}{l}\text { Haben Sie eine medizinische Ausbildung (z.B. Pflege, } \\
\text { Rettungsdienst, MTA, ...) absolviert? }\end{array}$ \\
\hline
\end{tabular}

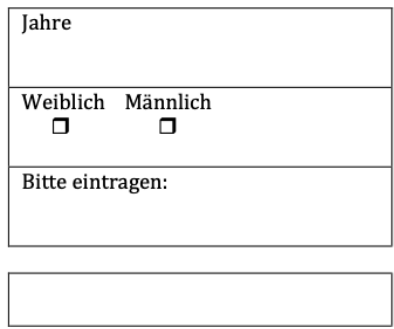

$\begin{array}{cc}J a & \text { Nein } \\ \square & \square\end{array}$

Bitte kreuzen Sie entsprechend an:

$$
\begin{array}{cccccc}
\text { Trifft gar nicht zu } & & & \multicolumn{3}{c}{\text { Trifft voll zu }} \\
\square & \square & \square & \square & \square & \square \\
\square & \square & \square & \square & \square & \square
\end{array}
$$

7. Der Kurs hat dazu beigetragen, dass ich Befunde besser beschreiben kann.

8. Wie beurteilen Sie folgende Ansätze, Ihre Fähigkeit zur Befundbeschreibung zu verbessern:

Keine Verbesserung Starke Verbesserung
$\begin{array}{llllll}\square & \square & \square & \square & \square\end{array}$
$\begin{array}{llllll}\square & \square & \square & \square & \square & \square\end{array}$
$\begin{array}{llllll}\square & \square & \square & \square & \square & \square\end{array}$
$\begin{array}{llllll}\square & \square & \square & \square & \square\end{array}$
$\begin{array}{llllll}\square & \square & \square & \square & \square & \square\end{array}$
$\begin{array}{llllll}\square & \square & \square & \square & \square\end{array}$ 
Meines Erachtens könnte das Dermatologie-Blockpraktikum verbessert werden durch:

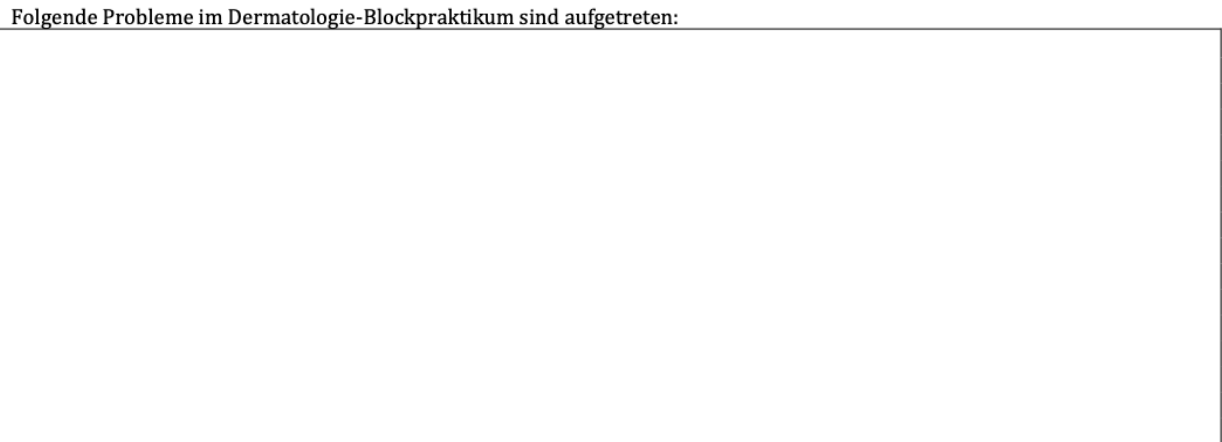




\title{
Anhang 9: Fragebogen zur Selbsteinschätzung (Experimentalgruppe)
}

\author{
Fragebogen zum „Epikrise Feedback“-Konzept im Dermatologie-Praktikums \\ Liebe Studierende, \\ wir versuchen Ihre Kompetenzen zur Bearbeitung dermatologischer Probleme durch \\ gegenseitiges Feedback zu verbessern. Bitte teilen Sie uns Ihre Meinung zu diesem Ansatz mit.
}

Datum:

2. Geschlecht

3. In welchem klinischen Semester befinden sie sich derzeit?

4. Haben Sie ein ähnliches Modell gegenseitigen Feedbacks schon einmal kennengelernt?

5. Wenn ja (bezogen auf Frage 2), in welcher Veranstaltung haben Sie diesen Ansatz kennengelernt?

6. Wie viele Famulaturen haben Sie bereits absolviert?

7. Haben Sie eine medizinische Ausbildung (z.B. Pflege, Rettungsdienst, MTA, ...) absolviert?

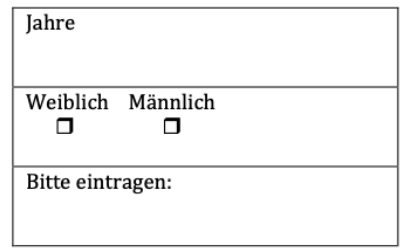

$\begin{array}{lc}\text { Ja } & \text { Nein } \\ \square & \square\end{array}$

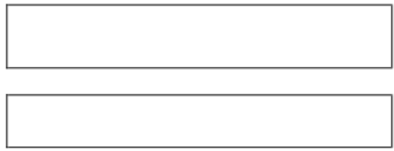

$\begin{array}{cc}\text { Ja } & \text { Nein } \\ \square & \square\end{array}$
Bitte kreuzen Sie entsprechend an:

8. Meine Motivation an dem gegenseitigen Feedback teilzunehmen war hoch

9. Das Feedback hat mich motiviert, mehr zu lernen.

10. Durch das Feedback hatte ich einen verstärkten Wissenszuwachs.

11. Das Konzept des gegenseitigen Feedback war verständlich

12. Die Benutzung der Lernplattform war verständlich.

13. Ich gebe dem Ansatz folgende Schulnote: ( 1 =sehr gut, 6 = ungenügend)

14. Bereits vor dem Kurs habe ich mir zugetraut Befunde zu beurteilen.

15. Der Kurs hat dazu beigetragen, dass ich Befunde besser beschreiben kann.

16

Durch das gegenseitige Feedback fühle ich mich sicherer bei der Beschreibung von Befunden.
Trifft nicht zu
Trifft voll zu
$\begin{array}{llllll}\square & \square & \square & \square & \square & \square\end{array}$
$\begin{array}{llllll}\square & \square & \square & \square & \square & \square\end{array}$
$\begin{array}{llllll}\square & \square & \square & \square & \square & \square\end{array}$
$\square \quad \square \quad \square \quad \square \quad \square \quad \square$
$\begin{array}{llllll}\square & \square & \square & \square & \square & \square\end{array}$
$\begin{array}{llllll}1 & \square & \square & \square & \square & 0\end{array}$
$\begin{array}{llllll}\square & \square & \square & \square & \square & \square\end{array}$
$\begin{array}{llllll}\square & \square & \square & \square & \square & \square\end{array}$
$\begin{array}{llllll}\square & \square & \square & \square & \square & \square\end{array}$ 
17. Wie beurteilen Sie folgende Ansätze, Ihre Fähigkeit zur Befundbeschreibung zu verbessern:

- Seminar

- Hausaufgabe

- Virtuelle Poliklinik

- Hospitation

- Schreiben der Epikrise

- Bed-Side Teaching

- Gegenseitiges Feedback
Keine Verbesserung Starke Verbesserung
$\begin{array}{llllll}\square & \square & \square & \square & \square & \square\end{array}$
$\begin{array}{llllll}\square & \square & \square & \square & \square & \square\end{array}$
$\begin{array}{llllll}\square & \square & \square & \square & \square & \square\end{array}$
$\begin{array}{llllll}\square & \square & \square & \square & \square & \square\end{array}$
$\begin{array}{llllll}\square & \square & \square & \square & \square & \square\end{array}$
$\begin{array}{llllll}\square & \square & \square & \square & \square & \square\end{array}$
$\begin{array}{llllll}\square & \square & \square & \square & \square & \square\end{array}$

Meines Erachtens hat das gegenseitige Feedback folgende Stärken:

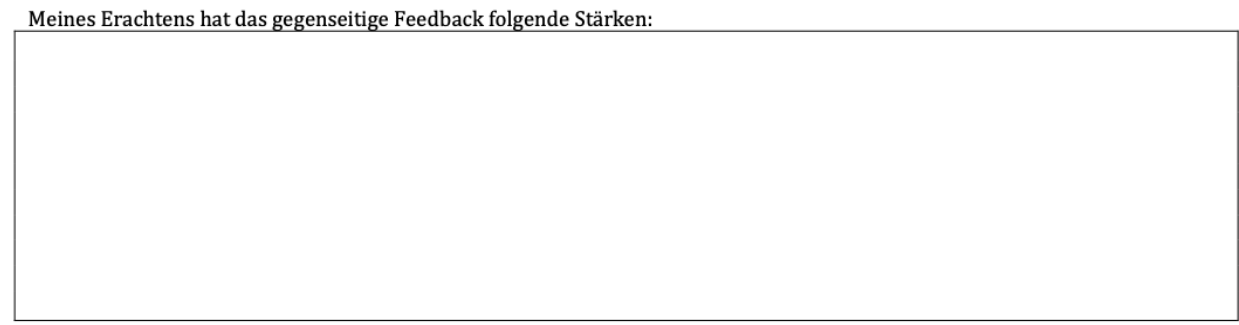

Meines Erachtens könnte das gegenseitige Feedback verbessert werden durch:

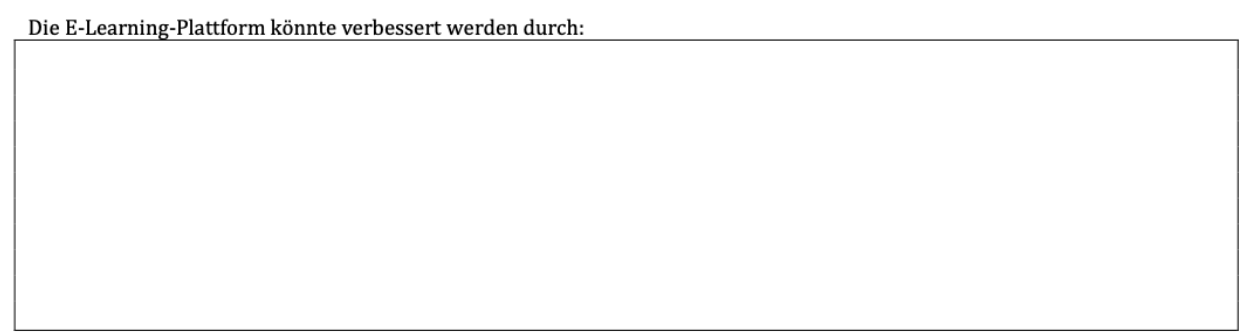

Folgende technische Probleme in der E-Learning-Plattform sind aufgetreten: 


\section{REFERENZEN}

1. Ochsendorf, F., Meister, L., Examination procedure and description of skin lesions. Hautarzt, 2017. 68(3): p. 229-242.

2. Ericsson, K.A., Deliberate practice and acquisition of expert performance: a general overview. Acad Emerg Med, 2008. 15(11): p. 988-994.

3. Norman, G.R., Coblentz, C.L., Brooks, L.R., Babcook, C.J., Expertise in visual diagnosis: a review of the literature. Acad Med, 1992. 92: $p$. 23-30.

4. Ochsendorf, F., Boehncke H., Beschmann H., Kaufmann R., Activation in clinical teaching with homework, questions of knowledge and feedback: experience in a dermatology practical course. GMS J Med Educ, 2007. 24(1).

5. Ochsendorf, F., Beschmann, H., Weberschock, T., Gille, J., Kaufmann, R., TIP-TOP Dermatology practical course: successful combination of classroom-learning und E-Learning. GMS J Med Educ, 2008. 25(1).

6. Ginsburg-Block, M., Rohrbeck, C., Fantuzzo, J., A meta-analytic review of social, self-concept, and behavioral outcomes of peer-assisted learning. Journal of Educational Psychology, 2006. 98(4): p. 732-749.

7. Field, M., Burke, J.M., McAllister, D., Lloyd, D.M., Peer-assisted learning: a novel approach to clinical skills learning for medical students. Med Educ, 2007. 41(4): p. 411-418.

8. Hattie, J., Timperley, H., The Power of Feedback. Review of Educational Research, 2007. 77(1): p. 81-112.

9. Wisniewski, B., Zierer, K., Hattie, J., The Power of Feedback Revisited: A Meta-Analysis of Educational Feedback Research. Front. Psychol., 2020. 10: p. 3087.

10. Reddy, S.T., Zegarek M.H., Fromme H.B., Ryan M.S., Schumann S.A., Harris I.B., Barriers and Facilitators to Effective Feedback: A Qualitative Analysis of Data From Multispecialty Resident Focus Groups. J Grad Med Educ, 2015. 7(2): p. 214-219.

11. Saedon, H., Salleh, S., Balakrishnan, A., Imray, C.H., Saedon, M., The role of feedback in improving the effectiveness of workplace based assessments: a systematic review. BMC Med Educ, 2012. 12(1): p. 25.

12. Norcini, J., Burch, V., Workplace-based assessment as an educational tool: AMEE Guide No. 31. Med Teach, 2007. 29(9): p. 855-871.

13. Wanjari, S., Feedback in Medical Education. JHSE, 2019. 5(2): p. 5357.

14. Van de Ridder, J.M.M., Stokking, K.M., McGaghie, W.C., Ten Cate, O., What Is Feedback in Clinical Education? Medical Education, 2008. 42(2): p. 189-197.

15. Stalmeijer, R.E., et al., Clinical teaching based on principles of cognitive apprenticeship: views of experienced clinical teachers. Acad Med, 2013. 88(6): p. 861-865.

16. Bing-You, R.G., Trowbridge, R.L., Why medical educators may be failing at feedback. JAMA, 2009. 302(12): p. 1330-1331.

17. Sender Liberman, A., Liberman, M., Steinert, Y., McLeod, P., Meterissian, S., Surgery residents and attending surgeons have different perceptions of feedback. Med Teach, 2005. 27(5): p. 470-472. 
18. Pelgrim, E.A., Kramer, A.W., Mokkink, H.G., Van der Vleuten, C.P., The process of feedback in workplace-based assessment: organisation, delivery, continuity. Med Educ, 2012. 46(6): p. 604-612.

19. Ende, J., Feedback in clinical medical education. JAMA, 1983. 250(6): p. 777-781.

20. Branch, W.T.J., Paranjape A., Feedback and reflection: teaching methods for clinical settings. Acad Med, 2002. 77(12 Pt 1): p. 11851188.

21. Najum, S.Q., Giving effective feedback in medical education. Education, 2017. 19(3): p. 243-248.

22. Cantillon, P., Sargeant J., Giving feedback in clinical settings. BMJ, 2008. 337: p. a1961.

23. Sumit, K.D., Henke P.K., Ailawadi G., Dimick J.B., Colletti L.M., Attending, house officer, and medical student perceptions about teaching in the third-year medical school general surgery clerkship. J Am Coll Surg., 2004. 199(6): p. 932-942.

24. Zehra, T., Tariq M., Ali S., Motiwala A., Boulet J., Challenges of providing timely feedback to residents: Faculty perspectives. Journal of the Pakistan Medical Association, 2015. 65: p. 1069-1074.

25. Han, E.R., Chung, E.K., Nam, K.I., Peer-Assisted Learning in a Gross Anatomy Dissection Course. PLoS One, 2015. 10(11): p. e0142988.

26. Van Zundert, M., Sluijsmans D., Van Merrienboer J.J.G., Effective peer assessment processes: Research findings and future directions. Learning and Instruction, 2010. 20: p. 270-279.

27. Huisman B., S.N., Van den Broek P., Van Driel J., The impact of formative peer feedback on higher education students' academic writing: a Meta-Analysis. Assessment \& Evaluation in Higher Education, 2019. 44(6): p. 863-880.

28. Ballantyne, R., Hughes K., Mylonas A., Developing procedures for implementing peer assessment in large classes using an action research process. Assessment \& Evaluation in Higher Education, 2002. 27(5): p. 427-441.

29. Tahir, I.H., A study on peer evaluation and its influence on college ESL students. Procedia-Social and Behavioral Sciences, 2012. 68: p. 192201.

30. Ilie, V., Frăsineanu, E.S., Traditional Learning versus E-Learning. EpSBS, 2019: p. 1192-1201.

31. McKimm, J., Jollie C., Cantillon P., ABC of learning and teaching: Web based learning. BMJ, 2003. 326(7394): p. 870-873.

32. Masic, I., E-learning as new method of medical education. Acta Inform Med, 2008. 16(2): p. 102-117.

33. Burnette, K., et al., Evaluation of a web-based asynchronous pediatric emergency medicine learning tool for residents and medical students. Acad Emerg Med, 2009. 16 Suppl 2: p. S46-50.

34. Cook, D.A., Levinson A.J., Garside S., Dupras D.M., Erwin P.J., Montori V.M., Internet-based learning in the health professions: a metaanalysis. JAMA, 2008. 300(10): p. 1181-1196.

35. Means, B., The Effectiveness of Online and Blended Learning: A MetaAnalysis of the Empirical Literature. 2013. 
36. George, P.P., et al., Online eLearning for undergraduates in health professions: A systematic review of the impact on knowledge, skills, attitudes and satisfaction. J Glob Health, 2014. 4(1): p. 010406.

37. Pei, L., Wu H., Does online learning work better than offline learning in undergraduate medical education? A systematic review and metaanalysis. Med Educ Online, 2019. 24(1): p. 1666538

38. Pandey, D., Pandey, V., Importance of Requirement Management : A Requirement Engineering Concern. International Journal of Research and Development, 2012: p. 2319-5479.

39. Khan, M., Ulhaq, S., Review of Requirements Management Issues in Software Development. International Journal of Modern Education and Computer Science, 2013. 5: p. 21-27.

40. Stellman, A., Greene, J., Applied Software Project Management. 2005: O'Reilly Media.

41. Royce, W., Managing the Development of Large Software Systems. Proceedings of IEEE WESCON, 1970. 26: p. 328-338.

42. Petersen, K., Wohlin, C., Baca, D., The Waterfall Model in Large-Scale Development. Product-Focused Software Process Improvement, 2009. 32: p. 386-400.

43. Benington, H.D., Production of Large Computer Programs. IEEE Annals of the History of Computing. IEEE Educational Activities Department. , 1983. 5(4): p. 350-361.

44. McConnell, S., Rapid Development: Taming Wild Software Schedules. 1996: Microsoft Press.

45. Oxagile. Waterfall Software Development Model. 2014 (abgerufen am 27.06.2020).

46. Hughey, D. Comparing Traditional Systems Analysis and Design with Agile Methodologies". University of Missouri - St. Louis. Retrieved 11 August 2014. 2009 (abgerufen am 27.06.2020).

47. Parnas, D.L., Clements, P.C., A rational design process: How and why to fake it. IEEE Transactions on Software Engineering, 1986: p. 251257.

48. McConnell, S., Code Complete. 2nd Edition. ed. 2004: Microsoft Press.

49. Bauch, R., Beer, T., Netzwerke - Grundlagen. 7. Auflage ed. 2004: HERDT-Verlag für Bildungsmedien.

50. Ferré, X., Juristo, N., Windl, H., Constantine, L., Usability Basics for Software Developers, in IEEE Software Magazine. 2001, IEEE Software. p. 22-29.

51. Budgen, D., Software Design. Second Edition ed. 2003: (C) AddisonWesley Publishers Limited 1993, (c) Pearson Education Limited 2003.

52. Avast $\circledast$. Avast $\circledast$ PC Trend Report 3. Quartal 2017| von Avast $\circledast 2.2017$ (abgerufen am 27.06.2020); Available from:

https://press.avast.com/hubfs/media-materials/kits/PC-trends-reportQ3-2017/DE/Avast-PC-Trends-Report-Q3-2017-DE\%20.pdf.

53. Avast $\AA$. Avast $\AA$ PC Trend Report 2019 | von Avast $\AA 2019$ (abgerufen am 27.06.2020); Available from:

https://cdn2.hubspot.net/hubfs/486579/Avast_PC_Trends_Report_201 9.pdf.

54. Technologies, A. Akamai's State of the Internet Q1 2014 Report (abgerufen am 29.06.2020). 2014. 7. 
55. Deutschland, S.G.-J.-B.-P.-H.-. Verfügbarkeit von Breitbandinternet $(\geq$ 1Mbit/s) für Haushalte in Deutschland nach Übertragungsart von 2010 bis 2018. 2020 (abgerufen am 27.06.2020); Available from:

https://de.statista.com/statistik/daten/studie/261287/umfrage/verfuegba rkeit-von-breitbandanschluessen-in-deutschland/\#professional.

56. Glushko, R.J., The Discipline of Organizing: Core Concepts Edition. 2014.

57. Sekikawa, A., Sa, E.R., Acosta, B., Aaron, D.J., Laporte, R.E., Internet mirror sites - The Lancet. Lancet, 2000. 355 (9219): p. 1923-2006.

58. Ltd., N.-. Web Server Survey. Netcraft, Ltd. 2020 (abgerufen am 25.06.2020).

59. Apache Software Foundation - Copyright $\odot 2019$ The Apache Software Foundation, L.u.t.A.L.V.A.a.t.A.f.I.a.t.o.T.A.S. Apache Software Foundation - Apache Licenses. 2019 (abgerufen am 25.06.2020); Available from: http://www.apache.org/licenses/.

60. Münch, I., Apache Webserver Sicherheitsstudie. 2003.

61. Castro, E., HTML for the World Wide Web. Subsequent ed. 2002.

62. Group, C.-T.P. PHP-Handbuch: Datenbankerweiterungen. 2001-2020 (abgerufen am 25.06.2020); Available from: https://www.php.net/manual/de/refs.database.php.

63. Group, C.-T.P. PHP-Handbuch: Erweiterungen. 2001-2020 (abgerufen am 25.06.2020); Available from: https://www.php.net/extensions.

64. Codd, E.F., A Relational Model of Data for Large Shared Data Banks. Communications of the ACM, 1970. 13(6): p. 377-387.

65. Codd, E.F., Derivability, Redundancy, and Consistency of Relations Stored in Large Data Banks. ACM SIGMOD Record, 2009 (1969). 38(1): p. 17-36.

66. Ambler, S. Relational Databases 101: Looking at the Whole Picture. 2002 (abgerufen am 27.06.2020); Available from: http://www.agiledata.org/essays/relationalDatabases.html.

67. Oracle-Corporation@2020-and/or-its-affiliates. What is MySQL? MySQL 8.0 Reference Manual. 2020 (abgerufen am 25.06.2020); Available from: https://dev.mysql.com/doc/refman/8.0/en/what-ismysql.html.

68. Limaye, M.G., Software Testing. 2009: Tata McGraw-Hill Education.

69. Saleh, K.A., Software Engineering. 2009: J. Ross Publishing.

70. Ammann, P., Offutt, J., Introduction to Software Testing. 2008: Cambridge University Press.

71. Everett, G.D., McLeod, R.J., Software testing-testing across the entire software development lifecycle. 2007.

72. Myers, G.J., The Art of Software Testing. Second Edition ed. 2004: John Wiley \& Sons, Inc., Hoboken, New Jersey.

73. Gao, J., Tsao, H.-S.J., Wu, Y., Testing and Quality Assurance for Component-based Software. Artech House. 2003.

74. Patton, R., Software Testing 2nd Edition ed. 2005: Indianapolis: Sams Publishing.

75. Scherer, T., Straub J., Schnyder D., Schaffner N., The effects of anonymity on student ratings of teaching and course quality in a bachelor degree programme. GMS Z Med Ausbild, 2013. 30(3). 
76. Donovan, J., Mader, C., Shinsky, J., Constructive student feedback: Online vs. traditional course evaluations. Journal of Interactive Online Learning, 2006. 5(3): p. 283-296.

77. Ravelli, B., Anonymous Online Teaching Assessments: Preliminary Findings. Annual National Conference of the American Association for Higher Education, 2000.

78. Deci, E.L., Koestner, R., Ryan, R.M., A meta-analytic review of experiments examining the effects of extrinsic rewards on intrinsic motivation. Psychol Bull, 1999. 125(6): p. 627-668; discussion 692-700.

79. Sopka, S., Beemelmanns, S., Rex, S., Beckers, S.K., Stieger, L., Rossaint, R., Marx, G., Peer-assisted learning in intensive care undergraduate teaching - concerning value increase for intensive care medicine. Anästh Intensivmed, 2015. 56: p. 206-215. 


\section{SCHRIFTLICHE ERKLÄRUNG}

Ich erkläre ehrenwörtlich, dass ich die dem Fachbereich Medizin der Johann Wolfgang Goethe-Universität Frankfurt am Main zur Promotionsprüfung eingereichte Dissertation mit dem Titel

Digital unterstütztes Peer-Feedback im Dermatologie-Praktikum

in der Klinik für Dermatologie, Venerologie und Allergologie unter Betreuung und Anleitung von Prof. Dr. Falk Ochsendorf mit Unterstützung durch Prof. Dr. Falk Ochsendorf ohne sonstige Hilfe selbst durchgeführt und bei der Abfassung der Arbeit keine anderen als die in der Dissertation angeführten Hilfsmittel benutzt habe. Darüber hinaus versichere ich, nicht die Hilfe einer kommerziellen Promotionsvermittlung in Anspruch genommen zu haben. Ich habe bisher an keiner in- oder ausländischen Universität ein Gesuch um Zulassung zur Promotion eingereicht. Die vorliegende Arbeit wurde bisher nicht als Dissertation eingereicht.

Luzern (Schweiz) 23.07.2020

(Ort, Datum)

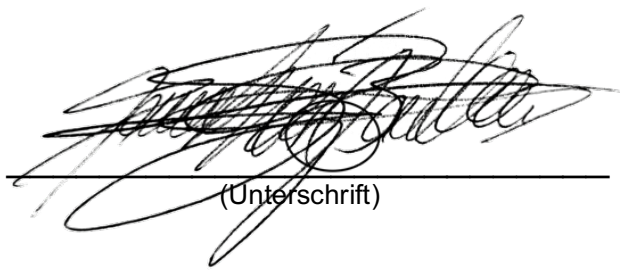




\section{DANKSAGUNG}

Herrn Prof. Dr. Falk Ochsendorf möchte ich für seinen Rat, seine Unterstützung und sein Vertrauen in mich danken. Dadurch habe ich mehr Interesse an der Forschung gewonnen und habe mein Wissen vertiefen können.

Ich möchte auch meinen Eltern und meiner Familie für alles danken. Ich kann nicht in Worte fassen, was ich innen alles verdanke.

Danke an alle, die ich liebe, liebte und lieben werde. Deren Unterstützung hat mich immer zum Erfolg gebracht. 


\section{LEBENSLAUF}

Der Lebenslauf wurde aus Gründen des Datenschutzes entfernt. 
